\title{
The role of Pcl5p and Pcl7p in the Gen4p stability regulation of Saccharomyces cerevisiae
}

\author{
Dissertation \\ zur Erlangung des Doktorgrades \\ der Mathematisch-Naturwissenschaftlichen Fakultäten \\ der Georg-August-Universität \\ zu Göttingen
}

vorgelegt von

Florian Schulze

aus

Uslar

Göttingen, 2010 

Die vorliegende Arbeit wurde in der Arbeitsgruppe von Prof. Dr. Gerhard H. Braus in der Abteilung Molekulare Mikrobiologie und Genetik des Instituts für Mikrobiologie und Genetik der Georg-August-Universität Göttingen angefertigt.

Teile dieser Arbeit wurden veröffentlicht in:

Streckfuss-Bömeke, K., Schulze, F., Herzog, B., Scholz, E., and Braus, G.H. (2009). Degradation of Saccharomyces cerevisiae transcription factor Gcn4 requires a C-terminal nuclear localization signal in the cyclin Pc15. Eukaryot Cell 8, 496-510.

D7

Referent: Prof. Dr. G. H. Braus

Korreferent: PD Dr. S. Irniger

Tag der mündlichen Prüfung: 27.10.2010 



\section{Table of contents}

TABLE OF CONTENTS........................................................................................................... I

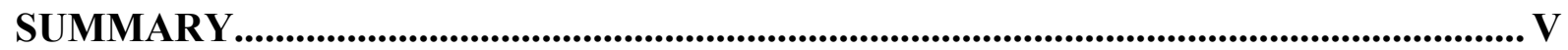

ZUSAMMENFASSUNG .........................................................................................................VI

1. INTRODUCTION .............................................................................................................. 1

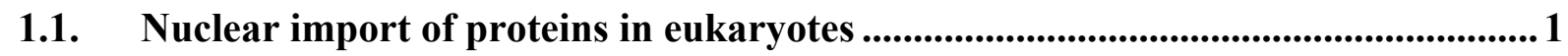

1.2. Ubiquitin ligases and ubiquitin-dependent protein degradation ..............................2

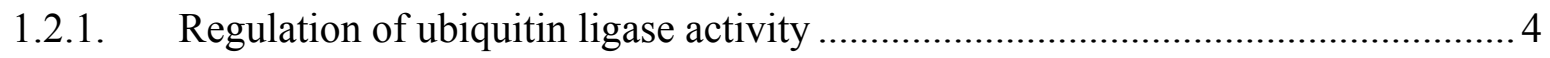

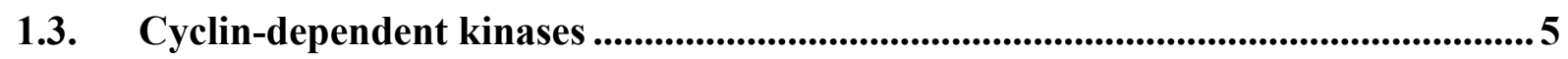

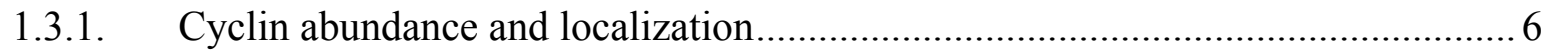

1.3.2. Regulation of CDKs through phosphorylation.................................................. 7

1.3.3. Regulation of CDKs by binding of inhibitors ............................................... 8

1.3.4. The CDK Pho85p and its interacting cyclins in S. cerevisiae............................. 9

1.3.5. Functions of Pho85p in cell cycle and morphogenesis of S. cerevisiae................ 9

1.3.6. Pho85p functions in metabolic processes....................................................... 11

1.4. Gcn4p and the general amino acid control network in $S$. cerevisiae .....................12

1.4.1. Induction of GCN4 expression in amino acid starved cells ................................ 14

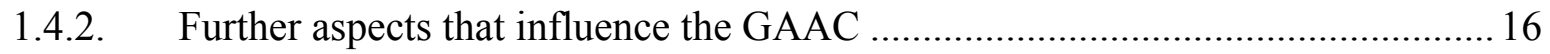

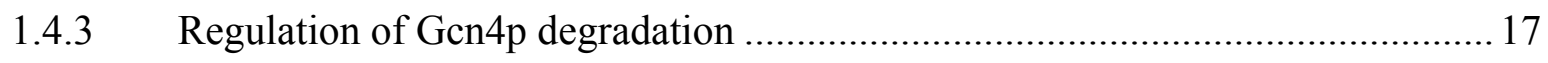

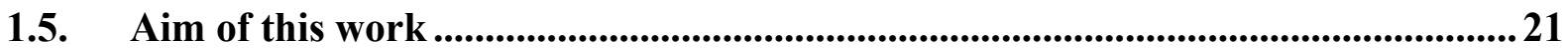

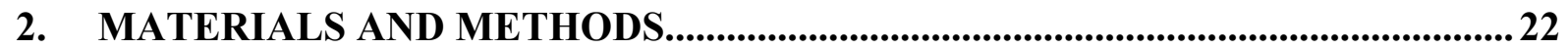

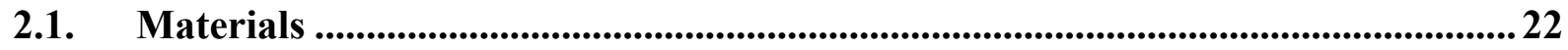

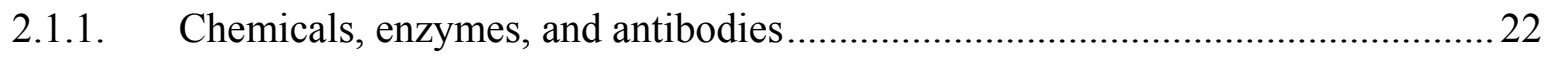

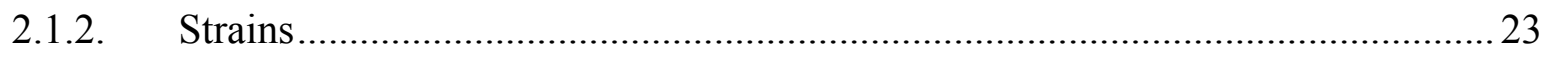

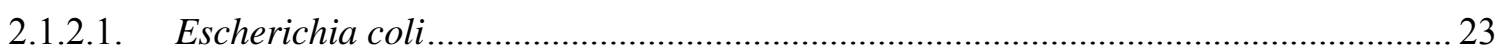

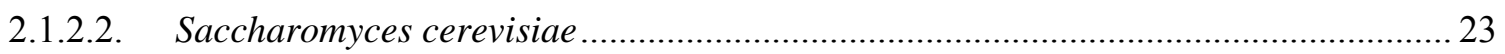

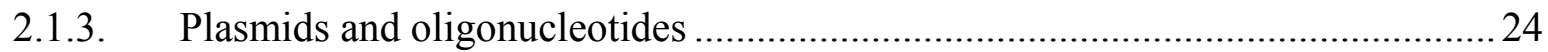

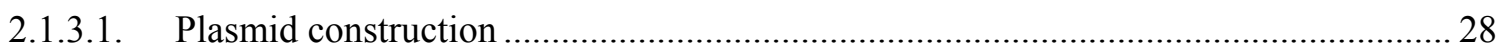




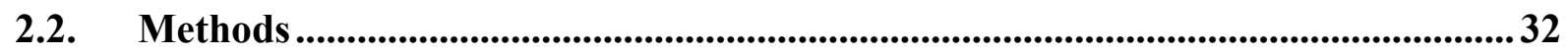

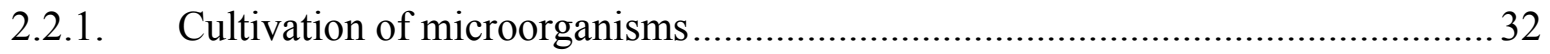

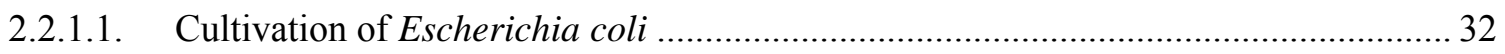

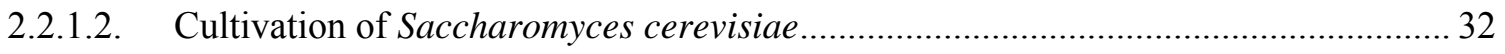

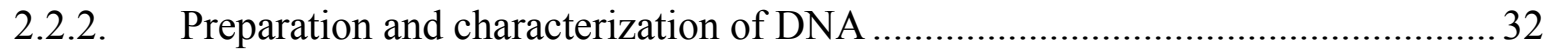

2.2.2.1. Quick boiling plasmid DNA preparation from E. coli ('STET prep') (Holmes and

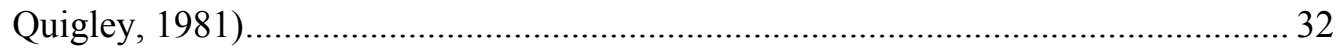

2.2.2.2. 'QIAprep spin miniprep' plasmid isolation from E. coli (QIAGEN, Hilden, D) ........... 33

2.2.2.3. Quick DNA preparation from yeast ('Smash \& Grab') ................................................ 33

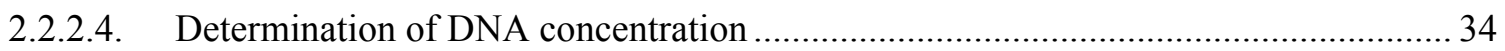

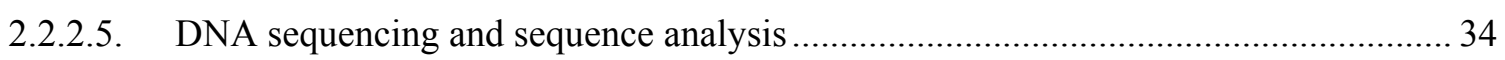

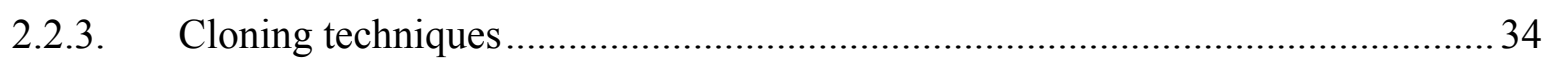

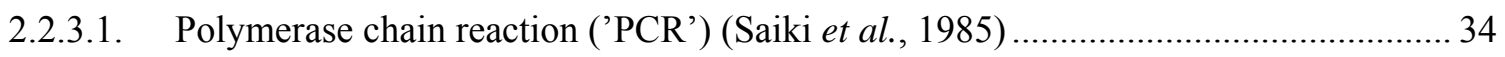

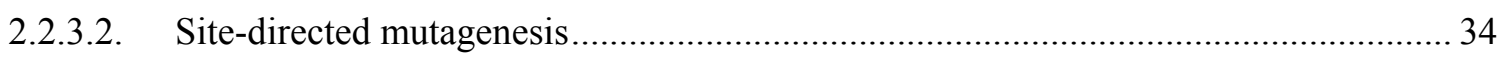

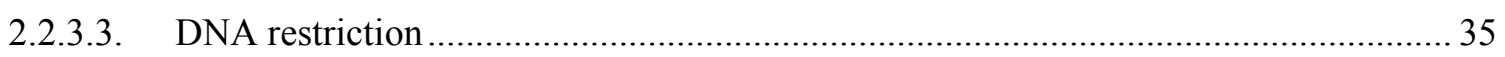

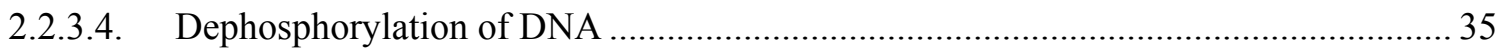

2.2.3.5. Ligation of DNA fragments (Maniatis et al., 1989) ................................................... 35

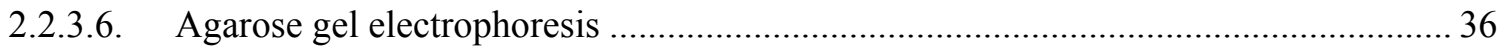

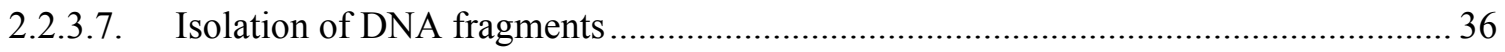

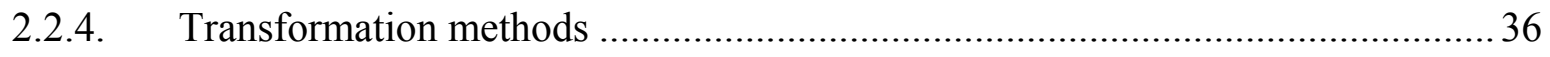

2.2.4.1. Preparation of chemically competent E. coli cells (Inoue et al., 1990)....................... 36

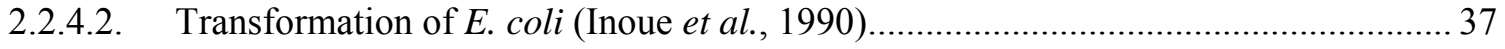

2.2.4.3. Transformation of S. cerevisiae by LiOAc method (Ito et al., 1983) ......................... 37

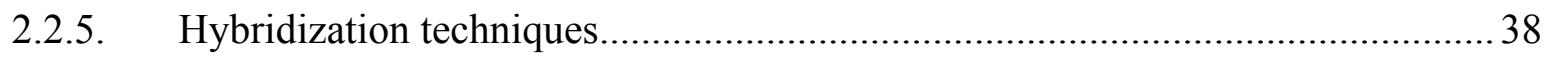

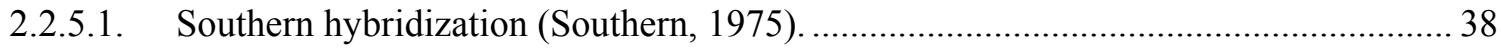

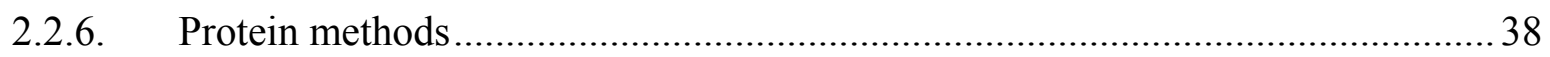

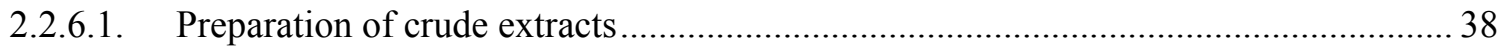

2.2.6.2. Determination of protein concentration (Bradford, 1976) .......................................... 39

2.2.6.3. SDS polyacrylamide gel electrophoresis 'SDS-PAGE' (Laemmli, 1970) .................. 39

2.2.6.4. Immunoblotting and detection of proteins (Towbin et al., 1979) ............................... 39

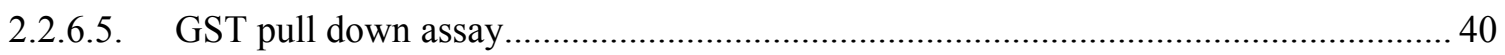

2.2.6.6. Protein stability assay ('Promoter shut-off' experiment) ............................................. 41

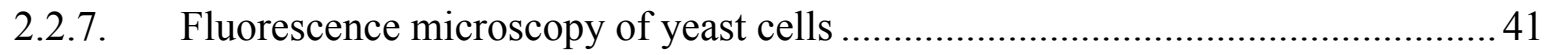

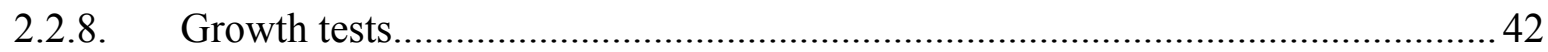


3. RESULTS

3.1. Characterization of the nuclear import of the cyclin Pcl5p in the yeast

Saccharomyces cerevisiae 43

3.1.1. A C-terminal NLS is required for proper Pc15p nuclear localization

3.1.2. Mutation of the C-terminal NLS motif in Pc15p partially impedes Gcn $4 p$ degradation in S. cerevisiae

3.1.3. The $\beta$ importin Kap95p imports Pcl5p into the nucleus.

3.1.4. A Pho80p-Pcl5p hybrid driven by the PCL5 promoter is functional to degrade Gen $4 p$

\subsection{Characterization of the Pho85p/Pcl5p interaction and the Gcn4p stability in} sated and amino acid starved cells of budding yeast

3.2.1. A Pho80-Pcl5-Pho80 hybrid interacts with Pho85p and Pho81p in the presence and absence of amino acids

3.2.2. The S. cerevisiae Pho85p/Pcl5p and Pho81p/Pcl5p complexes are formed independently of the amino acid availability

3.2.3. Constitutive formation of Pho85p/Pcl5p and Pho81p/Pcl5p complexes is independent of each other and does not require Pcl7p

3.2.4. Overexpression of PCL5 impairs the stabilization of Gen4p in amino acid starved yeast cells

3.2.5. Overexpression of PCL7 counteracts $\mathrm{Pho} 85 \mathrm{p} / \mathrm{Pcl} 5 \mathrm{p}$ activity .70

4. DISCUSSION .76

4.1. Pcl5p nuclear import requires a C-terminal NLS and is mediated by the $\beta$ importin Kap95p

4.2. The interaction of Pho85p and Pho81p with Pcl5p and the role of Pcl7p in the Gcn4p stability regulation

4.2.1. Five amino acids in Pc15p putatively mediate the interaction with Pho85p and Pho81p.

4.2.2. Pho85p/Pc15p and Pho81p/Pc15p complex formation is independent of the availability of amino acids

4.2.3. Competitive binding of Pcl7p to Pho85p counteracts Pho85p/Pcl5p complex formation

4.2.4. Model of the Gcn4p stability regulation in the yeast S. cerevisiae. .90 


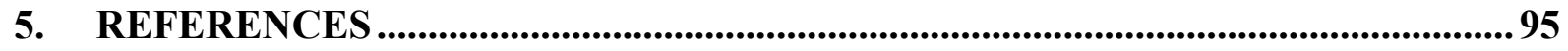

ACKNOWLEDGEMENTS ..................................................................................................... 105

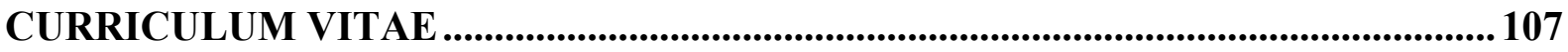




\section{Summary}

In the baker's yeast Saccharomyces cerevisiae, the transcriptional activator Gcn $4 p$ is the central element of the 'general amino acid control' network that induces more than 500 genes in response to amino acid limitation. The amount of Gcn4p in the cell is mainly regulated by a translational mechanism and via control of its stability. The high instability of Gen4p in sated cells is due to its rapid degradation via the ubiquitin-proteasome pathway which is initiated by phosphorylation through the cyclin-dependent kinase Pho85p in association with the cyclin Pcl5p. Pcl5p is a nuclear localized protein and its nuclear translocation depends on its C-terminus and was suggested to be mediated by the $\beta$ importin Kap95p. The amino aciddependent stabilization of Gcn4p in starved cells requires the cyclin-dependent kinase inhibitor Pho81p and another cyclin, Pcl7p. It was suggested that the initial step of the Gcn4p stabilization is the dissociation of the Pho85p/Pcl5p complex.

In the course of this study it was shown that the C-terminus of Pcl5p comprises a nonclassical NLS that requires a motif of basic amino acids flanked by a proline and an aspartic acid residue. The translocation of Pcl5p into the nucleus depends on a direct interaction with the $\beta$ importin Kap95p.

Truncated versions of Pc15p and Pc15p/Pho80p hybrids were used for interaction studies to identify regions of Pcl5p responsible for the assembly/disassembly of Pho85/Pcl5p and Pho81p/Pc15p complexes in sated and starved cells, respectively.

The investigations undertaken here confirmed that the middle part of Pcl5p is responsible for the substrate specificity towards Gen $4 p$ and suggest that this region also mediates the interaction with Pho85p and the cyclin-dependent kinase inhibitor Pho81p.

In addition, it was shown that Pc15p interacts with Pho85p and Pho81p independent of the amino acid availability. Furthermore, overexpression of PHO85, PCL5, and PCL7 indicate that the Gcn $4 p$ stabilization mainly depends on the acutely low amount of Pcl5p in starved cells. The stable cyclin Pcl7p has an indirect function in the Gcn4p stabilization by its competitive binding to Pho85p that counteracts the residual Pho85p/Pcl5p complex formation in starved cells. 


\section{Zusammenfassung}

In der Bäckerhefe Saccharomyces cerevisiae ist der Transkriptionsaktivator Gcn4p das zentrale Element der "Allgemeinen Kontrolle der Aminosäurebiosynthese”, der die Induktion von mehr als 500 Genen als Reaktion auf Aminosäuremangel bewirkt. Die Gcn4p-Menge in der Zelle wird überwiegend durch einen translationellen Mechanismus sowie durch Regulation der Stabilität kontrolliert. Die hohe Instabilität von Gcn4p in gesättigten Zellen beruht auf der schnellen Degradation über den Ubiquitin-Proteasom-Weg, die von der Phosphorylierung durch die Zyklin-abhängige Kinase Pho85p in Assoziation mit dem Zyklin Pcl5p initiiert wird. Pcl5p ist ein kernlokalisiertes Protein dessen Transport in den Kern von seinem C-terminus abhängig ist und vermutlich durch das $\beta$-Importin Kap95p vermittelt wird. Für die von der Aminosäureversorgung abhängige Stabilisierung von Gcn4p sind der Zyklinabhängige Kinase Inhibitor Pho81p, sowie ein weiteres Zyklin, Pcl7p, notwendig. Es wird angenommen, dass die Stabilisierung von Gcn4p durch die Dissoziation des Pho85p/Pcl5pKomplexes eingeleitet wird.

In dieser Arbeit konnte gezeigt werden, dass der C-Terminus von Pcl5p ein nicht klassisches NLS darstellt, das ein Motiv benötigt, in dem ein Prolin und ein Aspartat basische Aminosäuren flankieren. Der Transport von Pcl5p in den Kern erfolgt außerdem durch direkte Interaktion mit dem $\beta$-Importin Kap95p.

In dieser Arbeit wurden verkürzte Pcl5p-Varianten und Pc15p/Pho80p-Hybride verwendet, um Bereiche von Pcl5p zu identifizieren, die für die Assoziation/Dissoziation der Pho85p/Pcl5p-Komplexe, sowie Pho81p/Pc15p-Komplexe in gesättigten bzw. in hungernden Zellen benötigt werden. Die hier durchgeführten Untersuchungen bestätigen, dass der mittlere Bereich für die Substratspezifität verantwortlich ist und zeigen, dass dieser Bereich vermutlich auch die Assoziation mit Pho85p und Pho81p vermittelt.

Zusätzlich konnte gezeigt werden, dass Pc15p unabhängig von der Verfügbarkeit von Aminosäuren mit Pho85p und Pho81p interagiert. Darüber hinaus konnte durch Überexpressionsstudien gezeigt werden, dass die Stabilisierung von Gen4p hauptsächlich durch die geringe Menge von Pcl5p in hungernden Zellen vermittelt wird. Das stabile Zyklin Pcl7p hat hierbei nur eine indirekte Funktion. Die konkurrierende Bindung zu Pho85p wirkt der verbleibenden Pho85p/Pcl5p-Komplexbildung in hungernden Zellen entgegen. 


\section{Introduction}

\subsection{Nuclear import of proteins in eukaryotes}

The nucleus is characterized by an endoplasmatic reticulum associated membrane, which separates the cytoplasm from the nucleoplasm. This results in a spatial separation of translation and transcription and therefore requires a bidirectional intracellular trafficking of macromolecules such as proteins and RNAs between the two compartments. This regulated exchange takes place at the nuclear pore complexes (NPCs), which are elongated structures composed of nucleoporines in the nuclear envelope (Strambio-De-Castillia et al., 2010). While small molecules are able to pass the NPC by passive diffusion, proteins with a molecular weight of more than $40 \mathrm{kDa}$ need an active and signal mediated transport. The import of proteins depends on a nuclear localization sequence (NLS), whereas the export requires a nuclear export sequence (NES) in the cargo. Three classes of classical NLSs are known which share a high content of basic amino acids. The first class of NLS is similar to the monopartite NLS of the SV40 large tumor antigen consisting of a seven amino acid sequence with five basic amino acid residues (PKKKRKV) (Kalderon et al., 1984). The second type, the nucleoplasmin bipartite NLS (KRPAAIKKAGQAKKKK), is characterized by two clusters of basic amino acids that are separated by a region of 10-12 amino acids (Robbins et al., 1991). The third class of NLS consists of sequences with charged or polar amino acids separated by nonpolar amino acids like in the protooncogene c-myc in which a proline and an aspartic acid residue are flanking a basic region (PAAKRVKLD) (Makkerh et al., 1996). In contrast to the NLS, NES are less conserved and often rich in leucine residues (Kaffman and O'Shea, 1999).

Furthermore, the translocation requires GTP hydrolysis by the small GTPase Ran, soluble factors and specific transport proteins, the karyopherins, which recognize import or export signals within the cargo. In Saccharomyces cerevisiae 14 different karyopherins are known. Thirteen have been characterized as $\beta$ importins of which four are export receptors and nine import receptors. To the class of $\alpha$ importins belongs only Srplp (Hahn et al., 2008). The classical mechanism of nuclear import begins in the cytoplasm with binding of importin $\alpha$ to the NLS of the cargo protein. Then, complex formation with importin $\beta$ takes place mediated by the importin $\beta$ binding domain (IBB) of the $\alpha$ importin. This is followed by docking to the NPC, and subsequent translocation through the NPC into the nucleus. During this process importin $\beta$ remains at the NPC, whereas the complex of importin $\alpha$ and cargo protein enters 
the nucleus. After the release of the cargo into the nucleus the importins are transported out of the nucleus for the next round of transport (Figure 1). A non-classical nuclear transport without importin $\alpha$ also exists. In this case, the karyopherin of the importin $\beta$ family binds directly to the substrate, followed by docking of the complex to a subset of nucleoporins (Sorokin et al., 2007; Hahn et al., 2008; Fiserova and Goldberg, 2010).

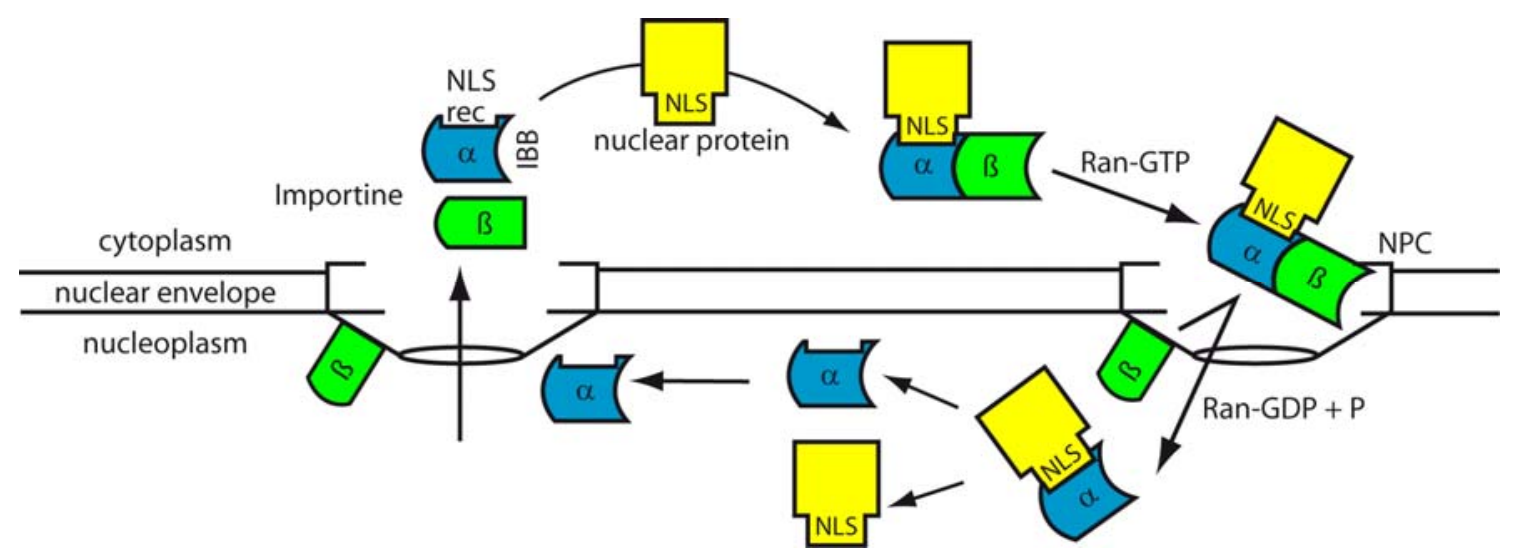

Figure 1: Model of nuclear protein import. The classical way of nuclear import of proteins requires an importin $\alpha / \beta$ complex and a nuclear localization sequence (NLS) in the cargo protein. First, importin $\alpha$ binds with its receptor domain (NLS rec) to the NLS motif of the cargo protein. This is followed by importin $\alpha / \beta$ complex formation mediated by the importin $\alpha$ located importin $\beta$ binding domain (IBB). In a process that requires GTP hydrolysis by Ran, the heterotrimeric complex enters the nuclear pore complex (NPC), which is mediated by an importin $\beta$ regulated docking. Importin $\beta$ dissociates and importin $\alpha$ with its bound cargo substrate enters the nucleoplasm. For the next round of nuclear import the importins have to return into the cytoplasm.

\subsection{Ubiquitin ligases and ubiquitin-dependent protein degradation}

Eukaryotic cells share a common mechanism to control protein degradation by the ubiquitinproteasome system (UPS). The system ensures that cellular key factors such as cyclins or transcription factors are degraded in a temporally and tightly regulated manner. Furthermore, the UPS is able to degrade mutated or misfolded proteins, which saves resources that can be used for other cellular events (Hershko, 2005; Hirsch et al., 2009). This degradation machinery includes the $26 \mathrm{~S}$ proteasome that specifically destructs proteins marked by the covalent attachment of a chain of the 76 amino acid protein ubiquitin. The covalent linkage of multiple ubiquitin molecules to the substrate is achieved in a three step enzymatic cascade (Figure 2). First, the ubiquitin is activated at the C-terminal glycine residue by the E1 activating enzyme in an ATP-dependent reaction. The activated ubiquitin is then transferred to an active cysteine residue of one of several E2 ubiquitin conjugating enzymes. Subsequently, ubiquitin is transferred to a $\varepsilon$-amino group of an internal lysine residue of the 
substrate by a member of the E3 protein ligase family. Attachment of further ubiquitins to a lysine residue (K48) of the previous ubiquitin molecule leads to a chain of poly-ubiquitin which is recognized by a specific receptor on the $26 \mathrm{~S}$ proteasome. The $26 \mathrm{~S}$ proteasome holoenzyme consists of a 20S core subunit with peptidase activity and two 19S regulatory particles with ATPase, chaperone, and deubiquitination activity, respectively. Before substrates are finally degraded by the $26 \mathrm{~S}$ proteasome into short peptides of approximately 325 amino acids, the ubiquitin is de-attached to be reused (Finley, 2009; Sorokin et al., 2009). Whereas only a single E1 enzyme is known, different types of E2s and E3s have been identified. In the baker's yeast, at least 13 genes were described encoding E2-like enzymes. E3 protein ligases are responsible for substrate recognition and it depends on the E3 ligase how the ubiquitin is passed to the substrate. E3 ligases are divided into two major groups: the HECT domain- and the RING finger-containing E3s. The RING finger-containing E3s do not covalently bind to the ubiquitin but mediate the transfer of the activated ubiquitin from the E2 enzyme to the substrate, whereas the HECT domain E3s bind to the activated ubiquitin before it is transferred to the substrate (Merlet et al., 2009). The majority of the RING-type E3s are multisubunit complexes such as the Anaphase Promoting Complex (APC) consisting of around a dozen subunits (Simpson-Lavy et al., 2010) or the modular cullin $\underline{\mathrm{R} I N G}$ finger ubiquitin ligases (CRL). CRLs are classified according to the cullin that functions as scaffold and which is required for the whole E3 complex formation. Cullins are conserved from yeast to mammals and in human there are at least seven cullins known (Cul1 to Cul7). In contrast, budding yeast only contains homologues of Cul1, Cul3, and Cul4, named Cdc53p, Cul3p, and Rtt101p, respectively (Merlet et al., 2009). The best characterized CRLs are Skp1-Cullin-ㅍbox (SCF) complexes that contain Cul1/Cdc53p. These SCF ligases generally consist of four

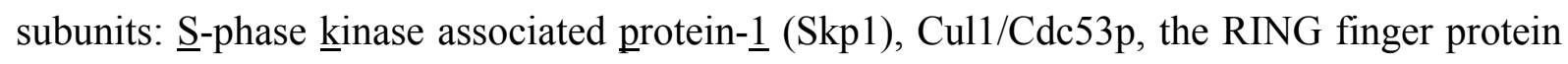
(Rbx1p, Roc1p, Hrt1p in yeast), and one of several F-box proteins (Figure 2). Rbx1 binds to the C-terminus of Cul1 and acts as linker to the ubiquitin-bound E2, whereas Skp1 mediates binding of the N-terminal part of Cull and the N-terminus of the substrate recruiting F-box protein. Furthermore, the substrate is bound to the C-terminal substrate binding domain of the F-box protein which recruits the substrate to the catalytic center of the core complex where the substrate is poly-ubiquitinated to be marked for proteasomal degradation as reviewed extensively (Bosu and Kipreos, 2008; Ravid and Hochstrasser, 2008; Deshaies and Joazeiro, 2009; Jonkers and Rep, 2009). 


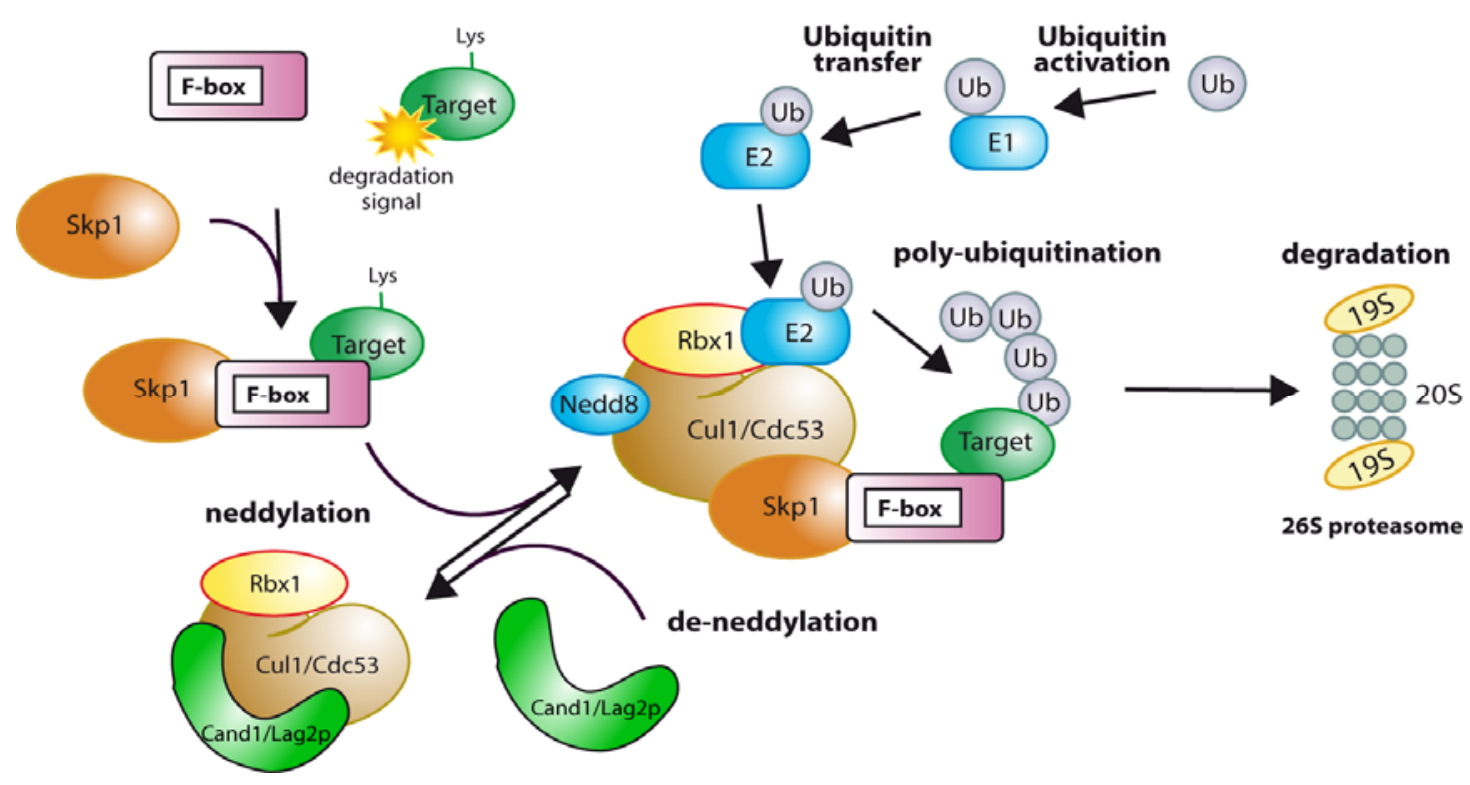

Figure 2: Scheme of the SCF complex and ubiquitin-dependent protein degradation. Ubiquitination of target proteins is mediated in a three step enzymatic cascade of a ubiquitin activating enzyme (E1), a ubiquitin conjugating enzyme (E2), and a ubiquitin ligase complex (E3). Polyubiquitinated substrates are degraded by the $26 \mathrm{~S}$ proteasome. The E3 ligase complex SCF is composed of four subunits: Skp1p, the cullin, the RING finger protein (Rbx1), and one of several substrate specific F-box proteins. Regulation of the SCF activity includes reversible conjugation of Nedd8 on the cullin subunit and the protein Cand1 (Lag2p in S. cerevisiae) (for details see 1.2.1.). Cand1/Lag2p binds unneddylated Cul1/Cdc53 leading to a stable inactive complex of Cand1/Lag2p-Cul1/Cdc53Rbx1. Binding of Skp1 in conjunction with an F-box protein facilitates the dissociation of Cand1/Lag2p. This initiates the SCF activation and the neddylation of the cullin takes place, which further inhibits binding of Cand $1 / \operatorname{Lag} 2 p$ and leads to conformational changes allowing the recruitment of activated E2. De-neddylation by the COP9 signalosome may then be required for inactivation of $\mathrm{SCF}$ and reformation of the inactive Cand1/Lag2p-Cul1/Cdc53-Rbx1 complex.

\subsubsection{Regulation of ubiquitin ligase activity}

The activity of RING E3 ligases is regulated by different mechanisms. A well known way of regulation is the modification of the substrate. Several substrates are specifically phosphorylated to change their affinity to the ubiquitin ligase. Examples in budding yeast are the cyclin-dependent kinase inhibitor Sic1p (Nash et al., 2001) or the transcription factor Gen $4 p$ that has to be phosphorylated by the cyclin-dependent kinases Pho85p and Srb10p to become a substrate of the ubiquitin ligase $\mathrm{SCF}^{\mathrm{CDC} 4}$ (Meimoun et al., 2000). Moreover, E2s and E3s can be affected by phosphorylation (Deshaies and Joazeiro, 2009). An additional regulatory mechanism is the alteration of the stability of the F-box proteins themselves which are degraded via an SCF- and ubiquitin-dependent autocatalytic mechanism that enables a rapid exchange in response to changing conditions. Furthermore, F-box mediated dimerization of SCF complexes was shown to increase its ligase activity (Bosu and Kipreos, 2008; Merlet et al., 2009). An important way to regulate the activity of CRLs is the reversible 
attachment of the small ubiquitin-like protein Nedd8 (Rublp in S. cerevisiae) to the cullin subunit. While neddylation is performed in an analogous E1, E2, and E3 enzyme cascade to the ubiquitin conjugating system, deneddylation is mediated by the CSN/COP9 signalosome. It is suggested that Nedd 8 together with Rbx 1 forms an active platform that increases the ubiquitin ligase activity through conformational changes of the cullin (Deshaies and Joazeiro, 2009; Merlet et al., 2009). Additional complexity within Nedd8-dependent regulation of CRLs is generated through the inhibitor protein Cand1 (Lag2p in S. cerevisiae) that binds to unneddylated cullins (Figure 2). Cand1 counteracts cullin neddylation and binding of substrate specific factors thereby inhibiting the activity and auto-ubiquitination of the SCF complex (Bosu and Kipreos, 2008; Merlet et al., 2009). Recently, it was shown that Drosophila-Cand1 prevents unneddylated cullin (Cul3) and its adaptor protein from proteasomal degradation (Kim et al., 2010). The budding yeast Skp1p was shown to require an associated F-box protein to efficiently remove the inhibitor Cand1/Lag2p from the cullin Cdc53p (Siergiejuk et al., 2009). It is proposed that Cand1/Lag2p binding to the cullin/Rbx 1 heterodimer blocks the assembly of the active CRL. This is counteracted by binding of Skp1 in complex with a substrate adapter (F-box protein) to the cullin promoting dissociation of Cand1/Lag2p which in turn allows neddylation of the cullin (Cul1/Cdc53p). This neddylation prevents re-association of the inhibitory Cand1/Lag $2 p$ and enables the recruitment of activated E2 and subsequent substrate ubiquitination. Inactivation of CRLs involves the deneddylation of the cullin by CSN/COP9 signalosome which is necessary to reform a stable Cand1/Lag2p-cullin complex (Siergiejuk et al., 2009).

\subsection{Cyclin-dependent kinases}

Cyclin-dependent kinases (CDKs) are involved in many cellular processes by phosphorylating substrates. The activity of CDKs requires an ancillary subunit, the cyclin, which also mediates the substrate specificity. Most cyclins posses the cyclin box domain (CB), a region of 150 amino acids with moderate sequence conservation. The $\mathrm{CB}$ was shown to form a typical five-helix structure with helix five contacting the CDK and helix one facing away from the CDK (Brown et al., 1995). Crystal structures of different CDKs including S. cerevisiae Pho85p bound to its cyclin Pho80p (Huang et al., 2007b) revealed that the interacting regions of cyclins and CDKs are different and vary in respect of involved loops and helices. The sites of the cyclin responsible for substrate recognition also vary. N-terminal, 
C-terminal, and cyclin box located sequences of cyclins have been shown to participate in substrate interactions (Huang et al., 2007b; Echalier et al., 2009; Lolli, 2010).

In $S$. cerevisiae six CDKs are known which are regulated by 23 cyclins. Like all CDKs, the yeast $\mathrm{CDK} /$ cyclin complexes are proline directed serine/threonine kinases and many substrates share the target consensus sequence S/TPXK/R. Cde28p and Pho85p are bound by various activating cyclins whereas the other four, Kin28p, Srb10p, Sgv1p, and Ctk1p, interact with only one cyclin (Liu and Kipreos, 2000). These four CDKs are all closely connected to gene expression through regulation of transcription and translation (Doonan and Kitsios, 2009). In contrast to higher eukaryotes, Cdc $28 p$ in S. cerevisiae is the only CDK essential for cell cycle events (Doonan and Kitsios, 2009). At the initiation of the cell cycle, Cdc28p is required for bud emergence and spindle pole body duplication in association with the G1 cyclins Cln1-3. In complex with the B-type cyclins Clb5 and Clb6, it is necessary for DNA replication and entry into $\mathrm{S}$ phase, whereas it is in complex with the later expressed cyclins Clb1-4 needed for mitosis. The CDK Pho85p can interact with ten different cyclins involved in many processes. This is the focus of sections 1.3.4, 1.3.5. and 1.3.6..

The activity of CDKs is tightly controlled by several mechanisms. As mentioned above CDK activation requires binding of cyclins, and their abundance and localization additionally generates specificity. The CDK/cyclin complex can undergo further regulatory mechanisms through phosphorylation or dephosphorylation or by binding of inhibitory proteins, the CDK inhibitors (CKIs).

\subsubsection{Cyclin abundance and localization}

The oscillating appearance and disappearance of cyclins is a well characterized way of CDK regulation and includes two mechanisms, the control of cyclin expression and cyclin proteolysis. In the baker's yeast, the periodic fluctuation of mRNA is best understood in case of the G1 cyclins that interact with Cdc28p. In the beginning of the cell cycle, CLN3 is transcribed and $\mathrm{Cdc} 28 \mathrm{p} / \mathrm{Cln} 3 \mathrm{p}$ complexes induce the transcription of the G1 cyclin encoding genes CLN1 and CLN2. Furthermore, the expression of CLB5 and CLB6 is induced with a peak during G1/S transition and the increasing amounts of these $\mathrm{Clb}$ cyclins lead to repression of other G1 cyclins and result in the activation of G2/M phase cyclins (Granovskaia et al., 2010; Suryadinata et al., 2010). The UPS mediated degradation of cyclins is necessary for cell cycle progression. The B-type cyclins Clb1-4 for example are required for mitotic events but prevent the exit from mitosis if not removed. Proteolysis of the mitotic B-type cyclins at the 
end of mitosis is mediated by the Anaphase Promoting Complex (APC) except for Clb6p (Irniger et al., 1995; Peters, 2006). In contrast, degradation of the G1 cyclins is not coordinated with the cell cycle and is SCF-dependend, similar to Clb6p, Cln $1 \mathrm{p}$ and Cln2p (Willems et al., 1996; Jonkers and Rep, 2009). Recently, it has been reported that the turnover of human cyclin A includes acetylation as novel regulatory mechanism (Mateo et al., 2009; Mateo et al., 2010).

The control of sub-cellular localization of cyclins is also important for the specific function of a CDK. For example, the G1 cyclin Cln3p primarily localizes in the nucleus and promotes transcription, whereas $\mathrm{Cln} 2 \mathrm{p}$ is predominantly localized in the cytoplasm at the bud neck and participates in polarized growth of budding yeast. A shift of $C \ln 3 p$ out of the nucleus into the $\mathrm{Cln} 2 \mathrm{p}$-like cytoplasmic localization allows $\mathrm{C} \ln 3 \mathrm{p}$ to fulfill some $\mathrm{Cln} 2 \mathrm{p}$-like functions (Miller and Cross, 2000). Similarly, in higher eukaryotes cyclin B1 is involved in reorganization of the cytoskeleton and cyclin B2, which normally participates in disassembly of the Golgi apparatus overtakes B1 function when directed to microtubules mediated by exchange of their N-terminal domains. (Draviam et al., 2001). This shows that the sub-cellular localization of a cyclin also influences CDK function.

\subsubsection{Regulation of CDKs through phosphorylation}

In addition to the regulation of CDK activity by cyclins, CDKs are also regulated by phosphorylation. Cyclin binding often leads to CDK phosphorylation promoting further conformational changes and full activation. Typical cell cycle CDKs like Cdc28p in budding yeast or CDK2 in human, are phosphorylated at conserved serine or threonine residues. Phosphorylation of Thr160 of CDK2 induces a conformational change creating the substrate binding site but also stabilizes the activated kinase as suggested for CDK2/cylin A, B, and E complexes (Brown et al., 1999; Barrett and Noble, 2005). In case of Pho85p/Pho80p in yeast or mammalian CDK5/p25, the phosphorylation is circumvented by a salt bridge between an aspartate residue from the cyclin and an arginine residue of the CDK (Tarricone et al., 2001; Huang et al., 2007b). However, phosphorylation can also have an inhibitory effect on kinase activity. Phosphorylation of Tyr15 of CDK2 in complex with cyclin A was suggested to decrease the kinase activity by reducing the affinity to its substrate (Brown et al., 1999). In S. cerevisiae the kinase Swe1p inhibits the activity of Cdc28p/Clb2p through phosphorylation of Cdc28p at Tyr19 which can be reversed by the phosphatase Mihlp. Interestingly, 
$\mathrm{Cdc} 28 \mathrm{p} / \mathrm{Clb} 2 \mathrm{p}$ also phosphorylates Swe1p which is thought to stabilize the inhibitory association of Swe1p to the CDK (Keaton et al., 2007; Hu et al., 2008).

\subsubsection{Regulation of CDKs by binding of inhibitors}

Further CDK regulators are the CDK inhibitors (CKIs) that switch off the catalytic activity of the kinases. In mammals two classes of CKIs are described, the INK4 family and the Cip/Kip family. Members of the INK4 family inhibit CDK activity by association with D-type cyclins that interferes with CDK/cyclin formation, whereas CKIs of the Cip/Kip family bind to both, cyclin and CDK and modulate cyclin D-, E, and B-CDK complexes (Pavletich, 1999; Lolli, 2010). In S. cerevisiae three CKIs have been identified, Pho81p, Sic1p, and Farlp (Mendenhall and Hodge, 1998). The latter is able to inactivate Cdc28p/Cln complexes and helps to stop the cell cycle at START in G1 and is important for mating induced growth arrest. Consistently, FAR1 transcription is induced by mating pheromone but its expression is cell cycle dependent and furthermore regulated via antisense RNA (Correia et al., 2010; Granovskaia et al., 2010). A further CKI that regulates the cell cycle in yeast is Sic1p inhibiting Cdc28p/Clb5,6 complexes. Siclp is regulated at the level of transcription and protein stability. It is stable until G1-S transition and is phosphorylated by the CDKs Cdc28p and Pho85p marking Siclp for $\mathrm{SCF}^{\mathrm{CDC} 4}$-mediated proteasomal degradation (Bloom and Cross, 2007; Jonkers and Rep, 2009). The third CKI in yeast, Pho81p, is required for Pho85p/Pho80p inhibition under low phosphate conditions although binding of Pho81p to the Pho85p/Pho80p complex occurs constitutively. The inhibitory function of Pho81p requires myo-D-heptakisphosphate (Lee et al., 2007; Lee et al., 2008) and its phosphorylation. This phosphorylation is mediated by the bound Pho85p/Pho80p complex and is a prerequisite for the tight association of Pho81p, mainly to the cyclin (Knight et al., 2004; Waters et al., 2004). Besides Pho85p/Pho80p complex inhibition, the CKI Pho81p is also involved in the stability control of the transcriptional activator Gcn4p, which is discussed in detail in section 1.4.3.

Other organisms were also shown to require CKI activity. In Schizosaccharomyces pombe Rum1p inhibits Cdc2p/Cdc13p to prevent mitosis whereas many roles of potential CKIs, like the Pho81p homologues AN4310 of Aspergillus nidulans or Afu4g06020 of A. fumigatus, remain unclear (Wu et al., 2004; Galagan et al., 2005; de Gouvea et al., 2008). 


\subsubsection{The CDK Pho85p and its interacting cyclins in S. cerevisiae}

The multifunctional CDK Pho85p interacts with ten cyclins and is involved in G1 progression. Furthermore, it also regulates cell polarity and the actin cytoskeleton, gene expression, glycogen and phosphate metabolism, and signaling of changes of the environmental conditions. Pho85p is the functional budding yeast homologue of the mammalian CDK5, which is involved in the development of the nervous system. Misregulated CDK5 activity has been implicated in several diseases like Alzheimer's and Parkinson's or amyothrophic lateral sclerosis. In addition, CDK5 participates in physiological functions and was shown to be involved in somatic disorders like diabetes mellitus (Chrousos and Kino, 2009; Lalioti et al., 2010; Lopes et al., 2010). Although Pho85p is not essential for viability, deletion of $\mathrm{PHO} 5$ causes a pleiotropic phenotype including hypersensitivity to many types of stress, abnormal morphogenesis, hyperaccumulation of glycogen, and constitutive expression of phosphate starvation dependent genes. Many of the various pho85 deletion phenotypes are complemented by overexpression of CDK5 in yeast. Several of these phenotypes are specific for one or more of the ten Pho85p cyclins (Pcls) which have been divided into two subfamilies according to sequence similarities within their cyclin-box region (Measday et al., 1997). The Pho80 subfamily includes the cyclins Pho80p, Pcl6p, Pcl7p, Pcl8p, and Pcl10p with significant functions in regulating metabolism and sensing environmental changes. The Pcl1,2-subfamily contains the cyclins Pcl1p, Pcl2p, Pc15p, Pc19p, and Clg1p. Except for Pcl5p, all members are primarily involved in regulation of cell cycle, cell polarity and morphogenesis (Figure 3) (reviewed by Huang et al., 2007a).

\subsubsection{Functions of Pho85p in cell cycle and morphogenesis of $S$. cerevisiae}

A well known role of Pho85p in cell cycle is in G1 phase when Pcl1p, Pcl2p, and Pc19p are specifically expressed. In association with the G1 cyclins Pcl1p and Pcl2p, Pho85p is required for cell cycle progression in the absence of the Cdc28p-activating cyclins $C \ln 1 p$ and $C \ln 2 p$ (Figure 3). Cells deleted for all four corresponding genes are inviable and arrest in G2 phase unable to pass the morphogenesis checkpoint. Cln $1 p$ and $C \ln 2 p$ and Pcllp and Pcl2p were shown to positively affect Cdc $24 p$, the GTP-GDP exchange factor for Cdc $42 p$ involved in cell polarity establishment (Moffat and Andrews, 2004). Furthermore, Pcl1p and Pcl2p direct Pho85p to positively regulate bud-site localization of Bni4p, an adaptor protein that targets 
several proteins to the bud neck (Zou et al., 2009; Larson et al., 2010) Additionally, the GTPase activating protein Rga2p, which is also involved in polar growth, is negatively regulated by Pho85p (Sopko et al., 2007). Moreover, Pho85p negatively influences the regulation of Rvs167p, an actin cytoskeleton protein required for endocytosis (Friesen et al., 2003).

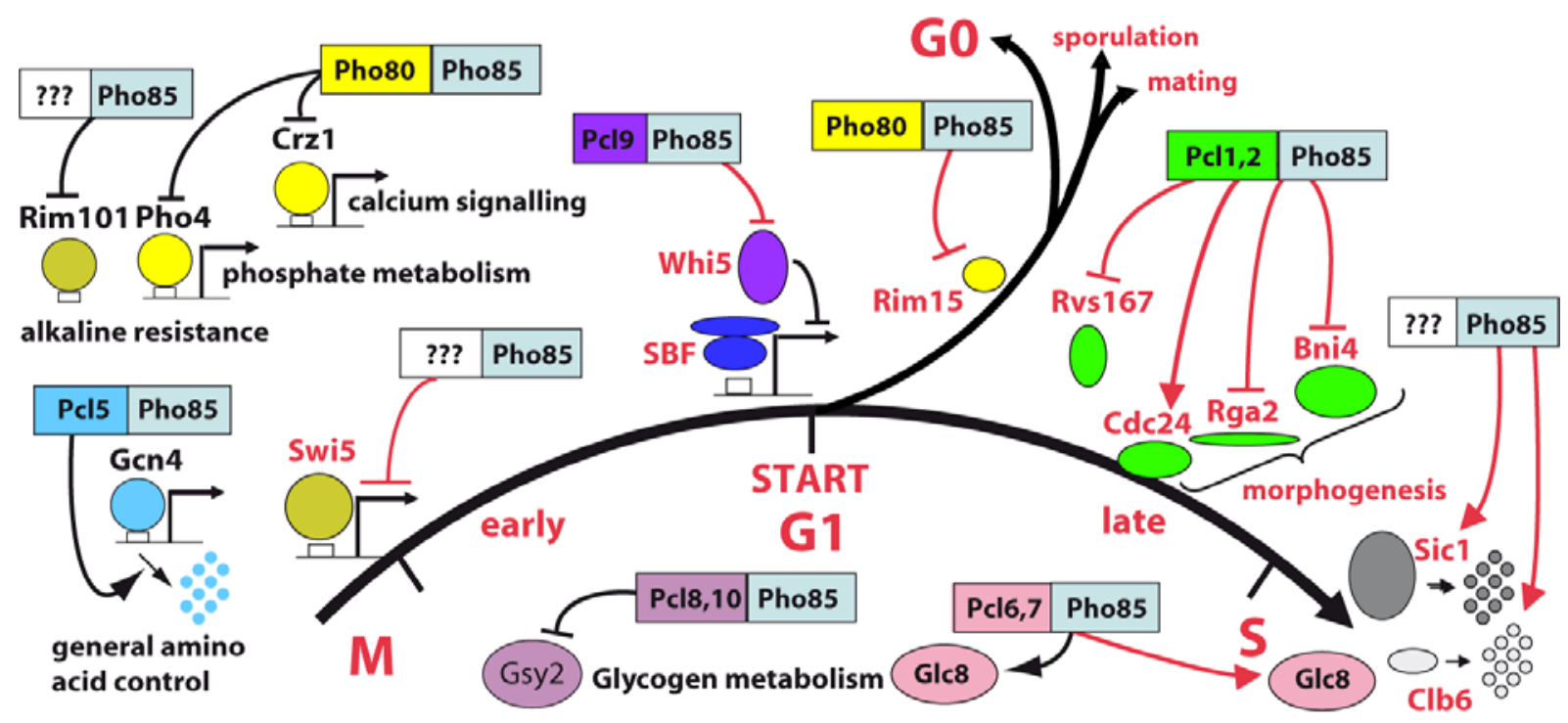

Figure 3: Overview of Pho85p functions (modified from Huang et al., 2007a). In complex with several different cyclins Pho85p is involved in many cellular processes. Black lines denote functions in response to environmental changes and red lines indicate functions of Pho85p in the cell cycle. Tbars represent negative regulation and arrows indicate positive influence of Pho85p activity. An arrow pointing to small circles shows degradation (for details see 1.3.5. and 1.3.6.).

In addition, Pho85p negatively regulates the transcription factor Swi5p which activates expression of genes in late M/early G1 phase, including PCL2, PCL9, the CKI encoding SIC1, and ASH1 (Measday et al., 2000). Recent data have shown that Pho85p/Pc19p directly targets the G1 specific transcriptional repressor Whi5p at G1/S phase transition. Phosphorylation of Whi5p by $\mathrm{Cdc} 28 \mathrm{p} / \mathrm{Cln} 3 \mathrm{p}$ as well as by Pho85p/Pc19p inhibits its activity by regulating the interaction of Whi5p with histone deacetylases and with the heterodimeric transcription factor SBF, thereby allowing SBF dependent transcription (Huang et al., 2009). Pho85p in association with Pc16p and Pc17p targets Glc8p, the regulatory subunit for Glc7p phosphatase required for mitotic spindle formation. In contrast to PCL6, the expression of PCL7 peaks during mid to late $\mathrm{S}$ phase which may be related to its role in activating Glc8p (Lee et al., 2000; Tan et al., 2003). In addition, Pho85p is involved in regulation of protein stability of the CKI Sic1p. Phosphorylation of the CKI Sic1p initiates its ubiquitin-dependent degradation and depends on $\mathrm{Cdc} 28 \mathrm{p} / \mathrm{Cln}$ kinases and on Pho85p whose activity is required to 
relieve yeast G1 checkpoint arrest after DNA damage when Cdc28p/Cln activity is downregulated (Nishizawa et al., 1998; Wysocki et al., 2006). Similarly, $\mathrm{SCF}^{\mathrm{Cdc} 4}$ mediated Clb6p degradation at the G1/S border requires phosphorylation by Cdc28p and Pho85p (Jackson et al., 2006). Moreover, Pho80p targets Pho85p to phosphorylate Rim15p, a protein kinase that is required in the nucleus for entry into G0 phase. Phosphorylation favors Rim $15 p$ association with Bmh2p in the cytoplasm, which leads to its exclusion from the nucleus when phosphate is available (Wanke et al., 2005; Smets et al., 2010).

\subsubsection{Pho85p functions in metabolic processes}

The CDK Pho85p has prominent roles in adaptation to nutrient availability mainly through activation of cyclins of the Pho80-subfamily. Pho80p, the best characterized cyclin of this subfamily, mediates specificity towards the Pho85p substrate Pho4p, a transcription factor that activates genes involved in phosphate metabolism and vacuole function. Under high phosphate conditions, the Pho85p/Pho80p kinase phosphorylates Pho4p while keeping it out of the nucleus. In contrast, Pho4p phosphorylation is decreased when phosphate becomes limited allowing hypophosphorylated Pho4p to enter the nucleus and to increase target gene expression whereas subsets of genes are activated under intermediary conditions. This regulation is complex and includes the binding and phosphorylation of the CKI Pho81p, myoD-heptakisphosphate and phosphorylation of different residues of Pho4p. Phosphorylation at two sites promotes the nuclear export whereas phosphorylation at a third site inhibits its nuclear import. Inhibition of the interaction with the transcription factor Pho2p is achieved by phosphorylation of a fourth residue (Komeili and O'Shea, 2000; Huang et al., 2007a; Lee et al., 2008). In addition, metabolic intermediates were shown to regulate the interaction of the transcription factors Pho4p and Pho2p (Pinson et al., 2009). Furthermore, it was shown that phosphate signaling is mediated via antisense and intragenic RNAs and that Pho4p activates and represses transcription also independently of the phosphate conditions (Nishizawa et al., 2008). Furthermore, Pho4p and the transcription factor Rim101p were shown to be necessary for the response to alkaline stress in a Pho85p dependent manner. The mechanism and the involved cyclins remain unsolved but Rim101p is negatively regulated by Pho85p leading to a more cytoplasmic localization (Nishizawa et al., 2010). Besides many other factors, the Pho85p/Pho80p kinase phosphorylates the transcription factor Crzlp, a mediator of the stress response to high levels of $\mathrm{Ca}^{2+}, \mathrm{Na}^{+}$and high temperature, leading to its cytoplasmic retention. Activation of Crzlp occurs through dephosphorylation under calcium dependent 
stress conditions by the phosphatase calcineurin (Sopko et al., 2006; Smets et al., 2010). The carbon source utilization and glycogen metabolism are also regulated by Pho85p. In association with the cyclins Pcl8p and Pc110p, Pho85p prevents hyperaccumulation of glycogen through inhibitory phosphorylation of the glycogen synthase Gsy $2 p$ and through repression of GSY2 transcription by an unknown mechanism (Huang et al., 1998; Wilson et al., 2010). Additionally, the cyclins Pcl6p and Pcl7p participate in glycogen storage mediating phosphorylation of Glc8p by Pho85p. This leads to activation of the Glc7p/Glc8p phosphatase that inactivates the glycogen phosphorylase Gphlp (Wilson et al., 2005). Deletion of PCL6 and PCL7 increases accumulation of glycogen but leads to defects in carbon source utilization. Moreover, the kinase activity of Pho85p/Pcl7p was shown to depend on the CKI Pho81p and on the availability of phosphate (Lee et al., 2000).

Another important feature that is regulated by Pho85p is the stability of Gen $4 p$, the key regulator of the general amino acid control. Gcn $4 p$ degradation depends on the availability of amino acids and is specifically initiated through Pho85p/Pcl5p mediated phosphorylation but its regulation also involves Pc17p and the CKI Pho81 (for further details see 1.4.4.).

In addition to the described functions of Pho85p in different cellular processes, its kinase activity can have antagonistically regulatory functions as shown for the tightly controlled process of autophagy. In association with the cyclins Pho80p and Pc15p, Pho85p negatively regulates autophagy through downregulating the kinase Rim15p and the transcription factors Pho4p and Gcn4p. However, the cyclins Clg1, Pcl1p, and Pho80p direct Pho85p to positively regulate autophagy by promoting the degradation of Siclp, which functions as negative regulator of autophagy that targets Rim15p (Yang et al., 2010).

\subsection{Gcn4p and the general amino acid control network in S. cerevisiae}

S. cerevisiae has evolved different interconnected networks for rapid and appropriate adaptation to changes in the environment like amino acid deprivation. A prerequisite to response to limiting conditions is sensing of the extra- or intracellular changes combined with signal transduction leading to proper modification of transcriptional, metabolic, or developmental programs. Therefore, most networks consist of a sensor kinase that mediates the response to the changing conditions and an activator that functions as downstream effector to induce the cellular response. A well characterized network that ensures the supply with amino acids is the 'general control of amino acid biosynthesis' (GAAC) in yeast that was first 
identified in the filamentous ascomycetes Neurospora crassa (Carsiotis et al., 1974) and A. nidulans (Piotrowska, 1980) where they were named 'cross pathway control' (CPC).

The key inducing element of the GAAC in the budding yeast is the transcription factor Gcn $4 p$ that is controlled at the level of transcription, translation and degradation and which is the functional homologue of A. nidulans CPCA protein. The mammalian counterpart is ATF4, which mediates the cellular response to different types of stress including amino acid deprivation and is involved in food selection, long-term memory, cell proliferation and differentiation (Costa-Mattioli et al., 2005; Franceschi et al., 2009; Kilberg et al., 2009).

Gcn $4 p$ consists of 281 amino acids and is the prototype of basic leucine zipper transcription factors. Leucine zippers (LZ) are motifs that are characterized by four repeats of leucine residues separated by seven amino acids and are important for dimerization (Landschulz et al., 1988). The 60 C-terminal amino acids of Gcn $4 p$ contain the LZ domain as well as the DNA binding domain sufficient for dimerization and DNA binding, respectively (Hope and Struhl, 1986). While Gcn $4 p$ binds to a specific 9 bp palindromic nucleotide sequence (5'$\left.\operatorname{ATGA}(\mathrm{C} / \mathrm{G}) \mathrm{TCAT}-3^{\prime}\right)$ as a homodimer, the mammalian homologous protein ATF4 can bind as homo- or heterodimer (Miller, 2009). Furthermore, Gcn4p harbours a domain for transcriptional activation that is spanning approximately half of the protein and is divided into a N-terminal and a central acidic activation domain with nearly identical activation potential (Drysdale et al., 1995). In addition, Gcn4p contains two nuclear localization sequences (NLS) that ensure constitutive nuclear translocation. NLS1 acts as ancillary signal and is not related to a canonical sequence, whereas NLS2 resembles a classical bipartite motif (Pries et al., 2002). Both motifs are sufficient for nuclear import but only NLS2 was shown to be conserved in other fungi. While the NLS2 mediated nuclear localization requires the importin $\alpha \operatorname{Srp} 1 \mathrm{p}$ and the importin $\beta$ Kap95p, the NLS2-dependent translocation is unspecifically mediated by several karyopherins (Pries et al., 2004).

Gcn $4 p$ activates the transcription of several hundred genes in response to various environmental stimuli (Natarajan et al., 2001; Kleinschmidt et al., 2005; Staschke et al., 2010). Under amino acid starvation conditions over 500 genes are upregulated in a Gcn4pdependent manner including genes encoding enzymes or co-factors involved in amino acid biosynthesis or nitrogen metabolism, genes encoding amino acid transporters, mitochondrial carrier proteins, or peroxisomal components as well as proteins involved in autophagy. Furthermore, Gcn $4 p$ has been shown to be necessary for the regulation of adhesion and filamentous growth in S. cerevisiae (Braus et al., 2003). Transcriptional profiling experiments of $\Sigma 1278 \mathrm{~b}$ cells, which are capable of adhesion, revealed five genes involved in this process 
that are expressed at higher levels in a Gcn4p-dependent way upon amino acid starvation including the flocculin encoding FLO11 (Kleinschmidt et al., 2005). In addition, PCL5, required for Gen $4 p$ degradation, is transcriptionally induced by Gen $4 p$.

\subsubsection{Induction of GCN4 expression in amino acid starved cells}

The amount of Gen4 protein in the cell is controlled by several mechanisms. Starvation for amino acids only slightly induces transcription of GCN4 resulting in two-fold increase of GCN4 mRNA after three to four hours of starvation (Albrecht et al., 1998). Gcn4p is mainly induced at the translational level (reviewed by Hinnebusch, 2005). Amino acid starvation activates the sensor of the GAAC network, the ribosome-attached kinase Gen2p by binding of uncharged tRNAs, which accumulate under these conditions, to the C-terminal histidyl-tRNA synthetase related domain (Wek et al., 1995). Moreover, this activitation requires the interaction of the N-terminus of Gen2p with the Gcn1p/Gcn20p regulatory complex via a Cterminal part of Gcn1p (Kubota et al., 2001; Sattlegger and Hinnebusch, 2005). This is further influenced by Yih1p, an inhibitor of Gen2p that competes, when overexpressed, for binding to Gcn1p thereby impairing the GAAC response (Sattlegger et al., 2004). Similarly, Gir2p together with Rbg1p were recently suggested to negatively influence Gen2p (Wout et al., 2009). Furthermore, the target of rapamycin (TOR) pathway negatively regulates Gen $2 p$ by promoting its phosphorylation at Ser577. This occurs indirectly by inhibition of the phosphatase Sit4p via Tap42p and is thought to decrease the affinity of Gen2p to uncharged tRNAs which is only important when their concentration is low (Cherkasova and Hinnebusch, 2003; Rohde et al., 2004). However, after binding of uncharged tRNA, the activated kinase domain of Gen $2 p$ phosphorylates the Ser51 residue of the $\alpha$-subunit of eIF2 bound to GDP (eIF2-GDP). Phosphorylated eIF2-GDP inhibits the guanine nucleotide exchange factor eIF2B resulting in a reduced concentration of eIF2 bound to GTP (eIF2-GTP). This, in turn, impedes formation of ternary complexes (TC) consisting of eIF2, GTP, and the charged methionyl initiator tRNA (tRNA ${ }^{\mathrm{MET}}$ ) which results in a reduced general translation but specifically stimulates GCN4 mRNA translation. The translational induction of GCN4 is caused by four short open reading frames (uORFs) in the 5' leader of the GCN4 mRNA with the first and fourth uORF being the most important ones. In non-starved cells high amounts of eIF2-GDP are recycled to eIF2-GTP by eIF2B, resulting in high concentration of TCs that form $43 \mathrm{~S}$ preinitiation complexes with the $40 \mathrm{~S}$ ribosomal subunit. These preinitiation complexes start scanning for the first AUG start codon near the 5' end of the GCN4 mRNA. 
At the first AUG of uORF I the 60S ribosomal subunit joins to form the 80S initiation complex and the translation starts. At the stop codon of uORF I the $80 \mathrm{~S}$ ribosome dissociates, leaving about half of the $40 \mathrm{~S}$ small ribosomal subunits attached to the mRNA. To further reinitiate translation, the eIF2-GDP must be recycled and the ternary complex has to be reformed, which happens before the uORF IV is reached. After translation of uORF IV the $80 \mathrm{~S}$ ribosome dissociates again preventing translation of the GCN4 ORF (Figure 4). In contrast, in amino acid starved cells the level of TCs is reduced due to less eIF2-GTP and 40S ribosomal subunits scan the region without rebinding of TCs and bypass uORF IV. This allows reinitiation at the next AUG start codon and leads to translation of the GCN4 ORF and thus, to an increase of Gcn $4 p$ under amino acid starvation conditions (Hinnebusch, 2005).

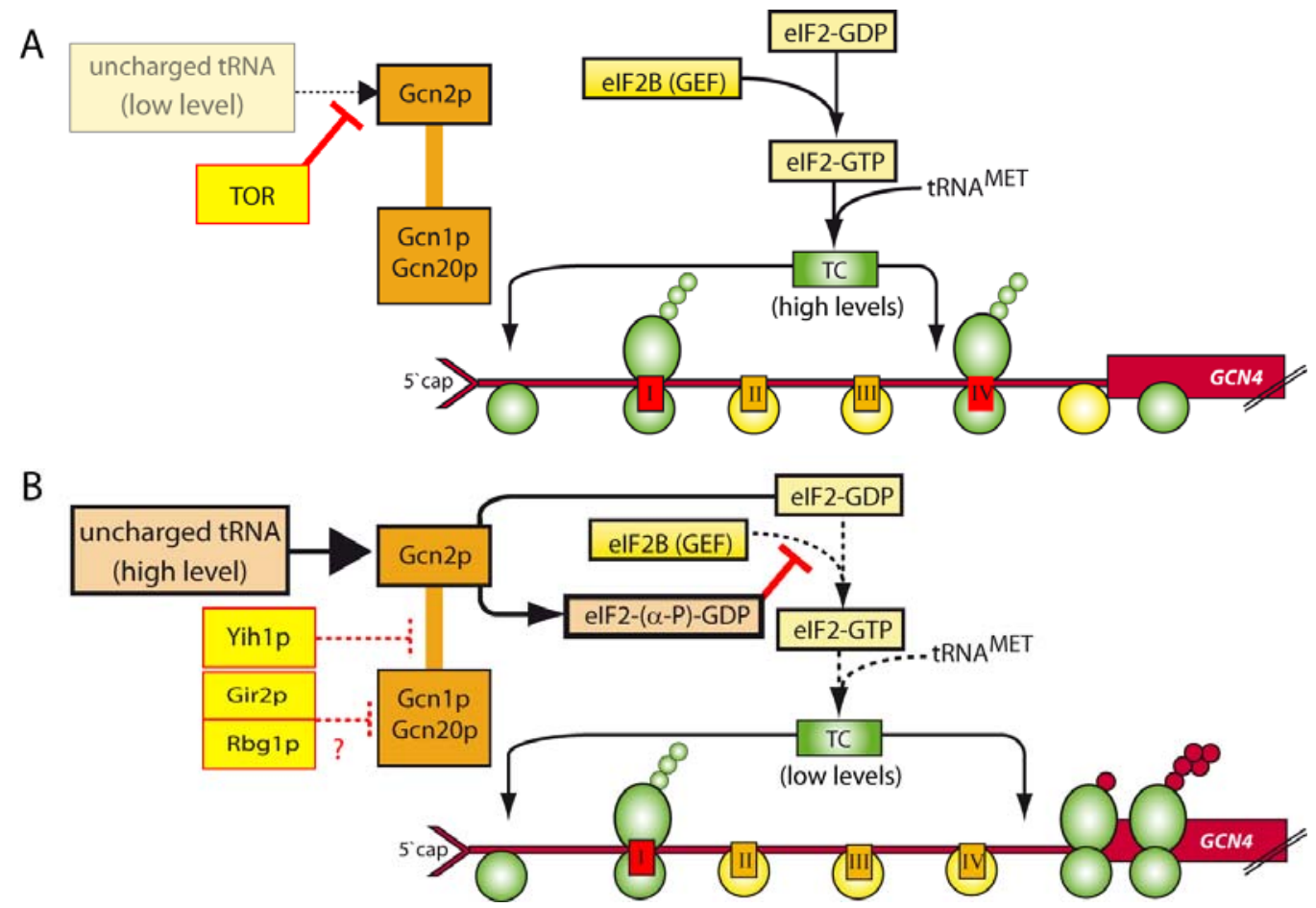

Figure 4: Regulation of GCN4 mRNA translation in S. cerevisiae (modified from Hinnebusch, 2005). (A) Under non starvation conditions four short open reading frames (uORF I-IV) prevent efficient translation of GCN4. Activation of the sensor kinase Gen2p requires binding of the Gcn1pGcn20p complex and uncharged tRNA. Gcn2p is not activated in sated cells by uncharged tRNAs due to their low concentration and the influence of TOR. Therefore, high amounts of eIF2 are recycled by the guanine exchange factor eIF2B resulting in high concentration of ternary complexes (TC) and the $80 \mathrm{~S}$ ribosome is formed at $\mathrm{uORF}$ I. After translation of $\mathrm{uORF}$ I, around $50 \%$ of the $40 \mathrm{~S}$ ribosomes remain and resume scanning. Due to the high amounts of TC, the 40S subunit rebinds the TC and translation is initiated again at uORF IV which prevents translation of GCN4. (B) Amino acid depletion leads to accumulation of uncharged tRNAs which activate Gen2p. Active Gen2p phosphorylates eIF2-GTP at subunit $\alpha$ which in turn leads to inhibition of eIF2B. Thus, less TCs are formed and reinitiation of translation is delayed. Therefore, reinitiation fails at uORF IV but occurs at GCN4 start codon. Activation of Gcn2p can be diminished by inhibitory binding of Yih $1 \mathrm{p}$ and maybe by the Gir2p-Rbg1p complex. For further details see text. 


\subsubsection{Further aspects that influence the GAAC}

In S. cerevisiae, the ribosome-attached sensor kinase Gcn2p functions indirectly as sensor of amino acid concentration through recognition of uncharged tRNA molecules which results in the activation of the GAAC as desribed above. GAAC activation is not mediated by growth on minimal medium since the baker's yeast has a sufficient high basal expression of amino acid biosynthesis genes to synthesize all 20 amino acids de novo. Therefore, the amino acid biosynthesis must be inhibited by the use of amino acid analogues like 3-amino-triazole (3AT) (Klopotowski and Wiater, 1965) or 5-methyl-tryptophan (5MT) (Schürch et al., 1974). Alternatively, the mutation of a biosynthetic enzyme resulting in an auxotrophic or a bradytrophic mutant can induce the GAAC response (Kornitzer et al., 1994). Besides this, there are other regulatory networks that are involved in sensing and responding to environmental changes and that influence the GAAC (Zaman et al., 2008; Smets et al., 2010). Further stimuli that directly or indirectly induce the GAAC response are the depletion of purines (Mösch et al., 1991), tRNA synthetases (Meussdoerffer and Fink, 1983), or glucose (Yang et al., 2000), as well as UV radiation (Engelberg et al., 1994), high salinity (Goossens et al., 2001), and treatment with the drug rapamycin (Valenzuela et al., 2001), the alkylating substance methyl methanesulfonate (MMS) (Natarajan et al., 2001) or $\mathrm{H}_{2} \mathrm{O}_{2}$ (Shenton et al., 2006) (Figure 5). All these stimuli lead to induction of GCN4 translation although a direct link to an increase of uncharged tRNAs or activation of Gen2p is not always clear. The activation of the GAAC by glucose depletion can be partially explained by reduced amino acid concentration in the cell observed under this condition. Furthermore, the kinase complex Snf1 plays a critical role in adaptation to glucose limitation and was recently shown to stimulate the GAAC response through activation of Gen $2 p$ but also by regulating eIF $2 \alpha$ phosphorylation independently of Gen2p (Shirra et al., 2008; Cherkasova et al., 2010). Likewise, a Gcn2p-independent induction of GCN4 translation was observed during a shiftdown from amino acid-rich to minimal medium and after UV irradiation that requires activation through protein kinase A (PKA) by an unknown mechanism (Tzamarias et al., 1989; Engelberg et al., 1994). Moreover, an increased affinity of Gen2p to uncharged tRNAs is a possible mechanism to induce the GAAC network, as mentioned above for the TOR pathway which is induced by rapamycin treatment but also upon nitrogen starvation (Zaman et al., 2008). Furthermore, it was suggested that TOR inhibits GCN4 transcription via inhibition of another signal transduction system, the nitrogen discrimination pathway (NDP) (Rohde et al., 2008). Recently, it was proposed that TOR regulates the GAAC to enable the 
utilization of secondary nitrogen sources (Staschke et al., 2010). In addition, the TOR pathway regulates the amino acid uptake by influencing the stability of different amino acid permeases in the membrane through a mechanism which involves the kinase Nprlp. The encoding gene, $N R P 1$, is, in turn, controlled by Gcn4p further exemplifying how complex these networks are converged (Smets et al., 2010).

Besides the regulation of GCN4 translation, the cellular amount of Gcn4p is also controlled at the level of protein degradation, which is the focus of the next section.

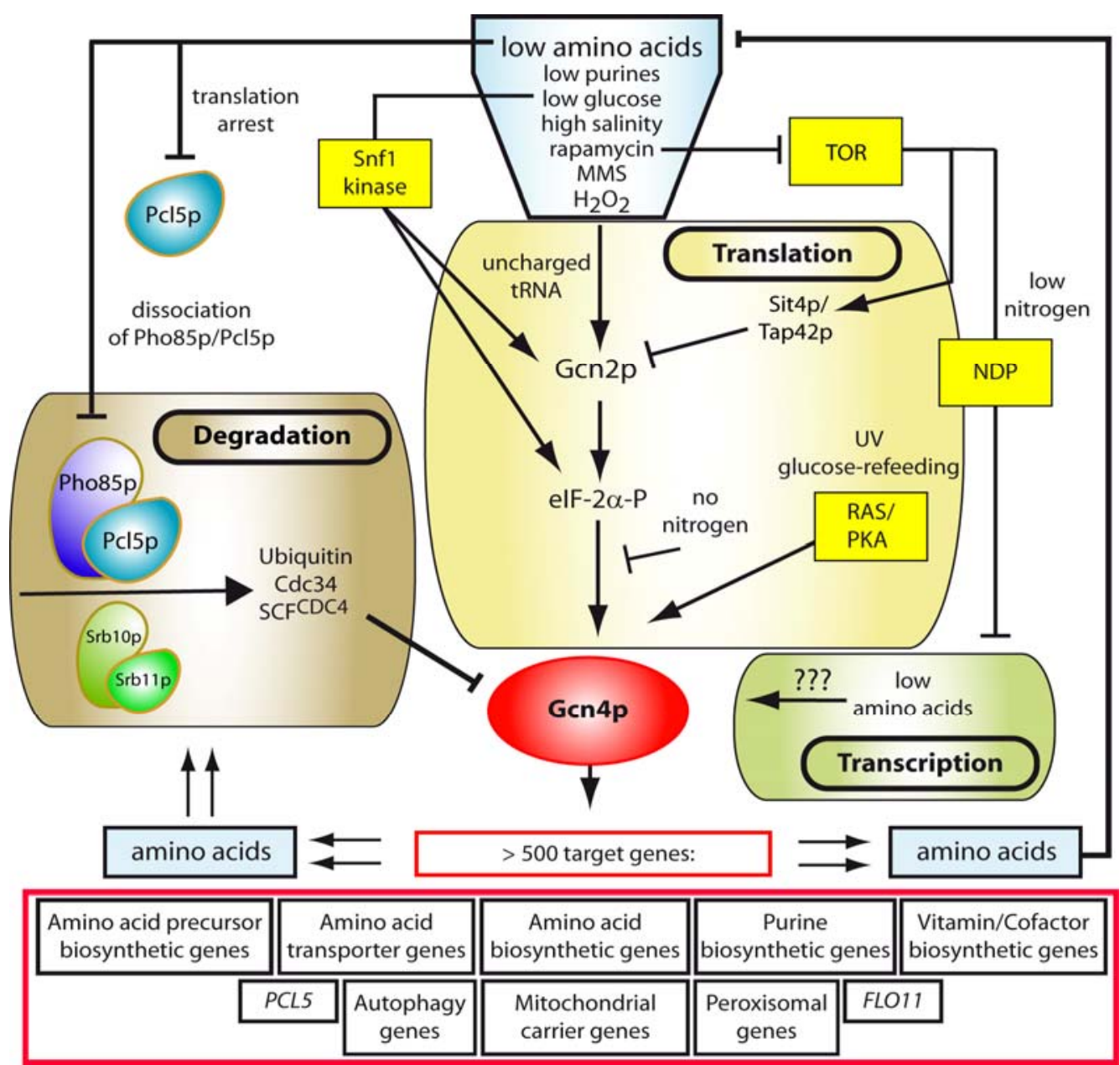

Figure 5: Summary of the general amino acid control (GAAC) network in S. cerevisiae. The transcription factor Gen $4 p$ is regulated in response to numerous environmental stimuli through control of its transcription, translation and protein stability. This, in turn, influences the induction of several hundred target genes that belong to different pathways (modified from Hinnebusch, 2005) (for details see text).

\subsubsection{Regulation of Gen4p degradation}

Under nutrient rich conditions, Gen4p is a short-lived protein with a half-life of only 3-4 minutes. However, in amino acid starved cells Gen $4 p$ degradation is slowed down leading to 
a half life of up to 20 minutes. To achieve Gcn $4 p$ stabilization, severe amino acid limitation is required. The stability of Gcn4p is only affected under conditions that lead to growth inhibition, like medium lacking the required amino acid of an auxotroph, high amounts of inhibitors such as $3 \mathrm{AT}$ in prototrophs or the translation inhibitor cycloheximide. Gcn $4 p$ contains a PEST region, which participates to the instability of the protein in concert with phosphorylation and ubiquitination sites. Thus, deletion of the PEST region leads to stabilization of Gen $4 p$. The rapid degradation of Gen $4 p$ in sated cells depends on phosphorylation by two CDK/cyclin complexes, Srb10p/Srp11p and Pho85p/Pcl5p. This phosphorylation marks the protein for poly-ubiquitination by the E2 enzyme Cdc34p together with the $\mathrm{E} 3 \mathrm{SCF}^{\mathrm{CDC} 4}$ RING finger ubiquitin ligase to become a target of the $26 \mathrm{~S}$ proteasome (Kornitzer et al., 1994; Meimoun et al., 2000). Therefore, impairment of one of both kinases leads to a stabilization of Gen4p (Meimoun et al., 2000; Chi et al., 2001). The Srb10p/Srb11p-dependent Gen $4 p$ phosphorylation is a constitutive process. Srb10p itself is a component of the mediator complex associated to the RNA polymerase II, which is required for activation of Gen $4 p$ target genes (Swanson et al., 2003; Zhang et al., 2004). Thus, the transcriptional activator Gcn $4 p$ might promote its own phosphorylation and subsequent destruction by recruitment of RNA polymerase II and Srb10p, which was suggested to be part of the promoter cleaning after transcription initiation (Chi et al., 2001). In contrast, the Pho85p initiated degradation of Gen $4 p$ is regulated dependent on the availability of amino acids and specifically requires binding of the cyclin Pcl5p. The increased stability of Gen $4 p$ in amino acid starved cells is due to less Pho85p/Pcl5p-dependent phosphorylation of the Gcn $4 p$ residue threonine 165 (Meimoun et al., 2000; Shemer et al., 2002). It was shown that Pho85p and Pcl5p dissociate under amino acid starvation conditions, which was supposed to initiate the Gcn4p stabilization (Bömeke et al., 2006).

The Gcn $4 p$ stability regulation takes place in the nucleus and Gcn $4 p$ as well as Pho85p are predominantly nuclear localized proteins independent of the amino acid availability (Pries et al., 2002). Furthermore, the Pho85p cyclin Pcl5p was shown to be constitutively nuclear localized and its exclusion from the nucleus in sated cells results in stabilized Gcn $4 p$ (Bömeke, 2006). similar to PCL5 deletion (Shemer et al., 2002).

Pcl5p consists of 229 amino acids and while its core 5-helix cyclin box domain (Figure 6) is responsible for substrate specificity towards Gcn4p (Aviram et al., 2008), its C-terminus has been shown to mediate nuclear localisation (Bömeke, 2006). With a half-life of only 2-3 minutes Pcl5p belongs to the Pcl1,2 family of instable Pho85p cyclins (Aviram et al., 2008). It contains two independent degradation signals (DS) ensuring the rapid and constitutive 
turnover of the protein, one in the N-terminal (NDS) and the other in the C-terminal part (CDS) of the protein. The latter requires a free carboxy end and is independent of Pho85p, whereas the NDS depends on Thr32 residue within a Pho85p consensus sequence that is autophosphorylated by the bound Pho85p kinase subunit leading to Pclp5 proteasmomal degradation. It was shown that this autophosphorylation induced degradation of Pcl5p is required for the GAAC response to amino acid starvation. Mutation of threonine 32 to alanine stabilizes Pcl5p leading to significantly reduced stabilization of Gcn $4 p$ in amino acid starved cells (Aviram et al., 2008). Furthermore, PCL5 was shown to be a target gene of Gcn4p and therefore PCL5 mRNA level is increased in amino acid starved cells (Jia et al., 2000). However, Pc15p does not accumulate under these conditions, which seems to be due to the reduced general protein biosynthesis in combination with the high instability of the protein (Shemer et al., 2002; Aviram et al., 2008).

A

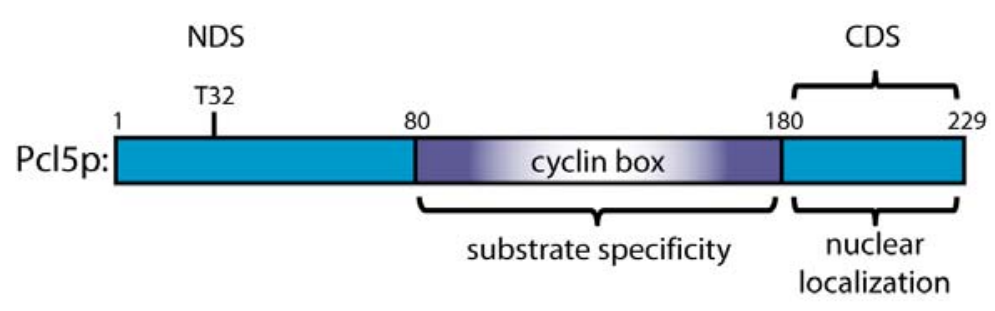

B

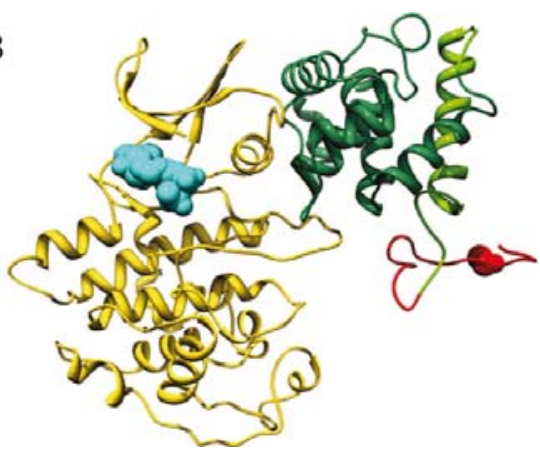

Figure 6: Composition of the 229-amino acid protein Pcl5p and predicted Pho85p/Pcl5p structure. (A) Instability of Pc15p is mediated by Thr32, which is part of a Pho85p-dependent Nterminal destruction signal (NDS) and by a C-terminal destruction signal (CDS) with unknown mechanism. The cyclin box domain mediates substrate specificity towards Gen $4 p$, whereas the Nterminus is responsible for proper nuclear transport. (B) This model is a prediction taken from Aviram et al., 2008 and includes residues 26 to 189 of Pcl5p. Pho85p is colored in yellow and the ATP- $\gamma$-S analogue as blue spheres. The Pcl5p cyclin box domain is depicted in dark green and the N-terminal domain in light green with the exception of residues 26 to 40 who are predicted to be disordered and colored in red. Thr32 is shown as red spheres.

Furthermore, the stabilization of Gen $4 p$ under amino acid depletion conditions requires another cyclin, Pcl7p, and the cyclin-dependent kinase inhibitor Pho81p, which are both predominantly nuclear localized (Bömeke et al., 2006). Deletion of either PCL7 or PHO81 inhibits Gcn $4 p$ stabilization in starved cells and leads to rapid degradation of the transcription factor under conditions when it is normally stabilized (Bömeke et al., 2006). It was shown that the interaction of Pcl7p with Pho85p and Pho81p is independent of the presence or absence of amino acids. However, the Pho85p/Pcl5p complex and also the interaction of 
Pho81p with Pcl5p was disrupted under amino acid starvation conditions (Bömeke et al., 2006).

Based on these data, the following model was suggested (Figure 7): Pho85p/Pcl5p and Pho81p/Pcl5p complexes are in balance with Pho85p/Pcl7p and Pho81p/Pcl7p complexes in sated cells. In amino acid starved cells the Pho85p/Pc15p and Pho81p/Pcl5p complexes dissociate and Pcl5p disappears from the cells because of its rapid turnover and overall reduced translation. This leads to a shifted balance towards more Pc17p containing complexes and less Pcl5p containing complexes resulting in reduced Gcn4p degradation and therefore stabilization of the transcription factor Gen4p (Bömeke et al., 2006).
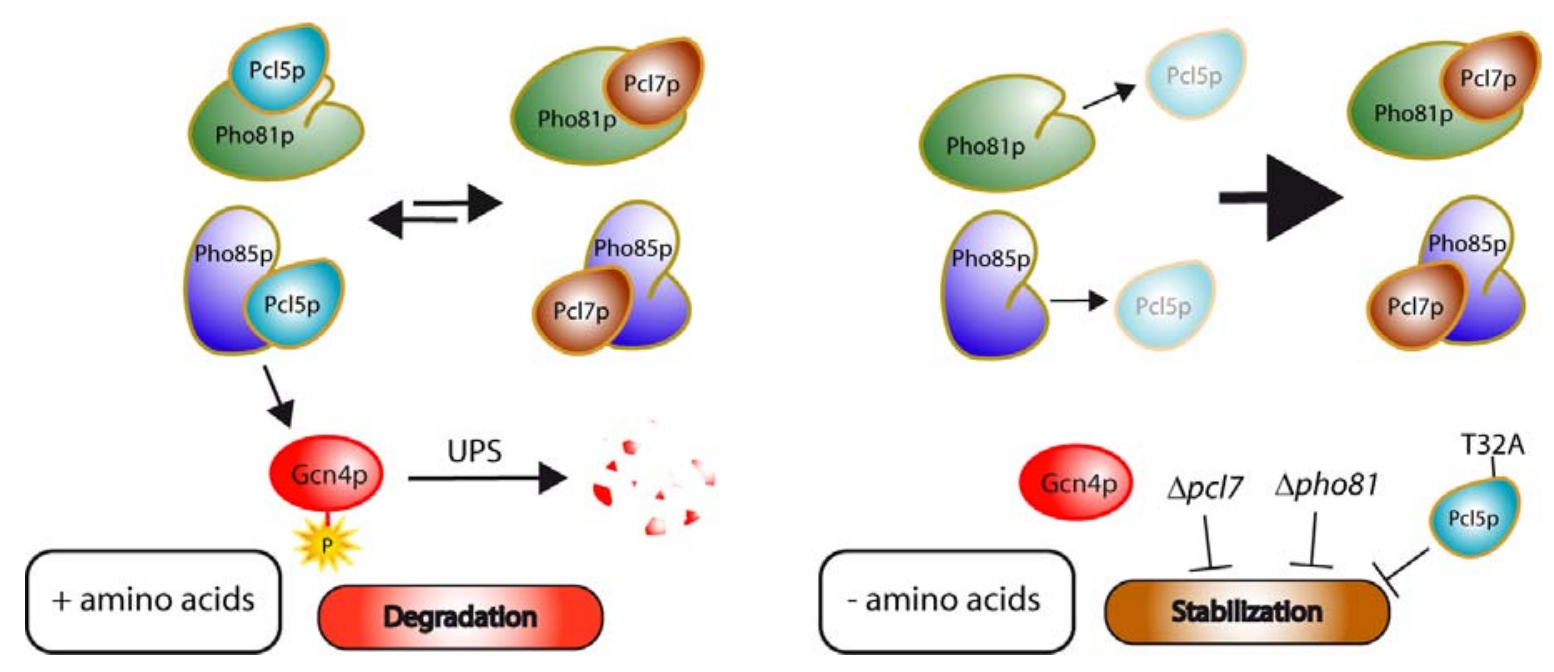

Figure 7: Model of Gen4p stability regulation. Under conditions of amino acid availability, Pho85p/Pc15p and Pho81p/Pcl5p complexes are in equilibrium with Pho85p/Pcl7p and Pho81p/Pcl7p complexes and Pho85p/Pcl5p initiate the ubiquitin-proteasome (UPS) mediated degradation of Gcn $4 p$ by phosphorylation. In amino acid depleted cells, this equilibrium is shifted to Pcl7p containing complexes due to the dissociation of Pcl5p from Pho85p and Pho81p combined with the rapid Pcl5p turnover and its decreased translation under this condition. Therefore, less Gcn $4 p$ is phosphorylated and targeted for degradation leading to its increased stability. This stabilization is impaired when Pcl5p is stabilized due to Thr32Ala substitution or in cells deleted for PCL7 or PHO81. For details see text.

In addition, it was shown that Pcl7p is not involved in the dissociation of Pho85p/Pcl5p or Pho81p/Pcl5p complexes but it was suggested that Pcl7p binding counteracts the Pho85p/Pcl5p interaction. Moreover, Pho85p is not required for the Pho81p/Pcl5p interaction in sated cells or their dissociation in starved cells. Similarly, the CKI Pho81p is not required for the interaction of Pho85p/Pcl5p. Surprisingly, Pho81p seems to be required to obtain a certain Pcl5p level in amino acid starved cells, although no direct interaction between Pho81p and Pcl5p has been observed under this condition. This is contradictory to the observed 
decreased stabilization of Gcn $4 \mathrm{p}$ in amino acid starved cells lacking PHO81 since reduced Pho85p/Pc15p complexes are predicted to increase the Gcn4p stability (Bömeke et al., 2006).

\subsection{Aim of this work}

The transcription factor Gcn $4 p$ is the key activator of the general amino acid control network in S. cerevisiae and is translationally induced by several stimuli. Furthermore, Gcn $4 p$ is regulated on the level of. protein stability in an amino acid-dependent manner and this occurs exclusively in the nucleus (Pries et al., 2002). The highly unstable cyclin Pcl5p was shown to be specific to direct the cyclin-dependent kinase Pho85p to mark Gen $4 p$ for degradation (Shemer et al., 2002). Moreover, Pcl5p nuclear localization was shown to be one prerequisite for the Pho85p-dependent initiation of Gcn $4 p$ degradation (Bömeke, 2006). It was proposed that the middle part of Pcl5p containing the cyclin box domain confers substrate specificity towards Gcn4p, whereas the C-terminus of Pcl5p together with the karyopherin Kap95p was suggested to mediate its nuclear localization (Bömeke, 2006). One aim of this study was to get more insights into the nuclear transport of the cyclin. Therefore, the influence of a putative nuclear localization signal on Pcl5p localization and Gen $4 p$ degradation was analyzed in the context of different truncated Pcl5 hybrids fused to GFP. Furthermore, the impact of the importin Kap95p on Pc15p nuclear localization was investigated in more detail.

The second aim of this study focuses on the amino acid-dependent Gen $4 p$ stability regulation. Several proteins were shown to be involved in this tightly controlled process. It is assumed that the disassembly of the Pho85p/Pcl5p complexes under starvation conditions together with the high instability of Pcl5p lead to the stabilization of Gcn $4 p$ in starved cells. This stabilization requires the cyclin-dependent kinase inhibitor Pho81p and another Pho85p cyclin, Pcl7p (Bömeke et al., 2006). The mechanism of how Pcl7p or Pho81p participate in stabilization is unknown. Thus, the aim of this project was to elucidate the role of Pcl7p and Pho81p or putative post-translational modifications of the cyclin Pcl5p in the mechanism of Pho85p/Pcl5p association or dissociation and hence, of Gen $4 p$ stability regulation. In this work, interaction studies of different Pc15p hybrids with Pho85p and Pho81p were performed to identify Pc15p domains or modification sites responsible for assembly or disassembly of the complexes. Furthermore, the impact of high amounts of Pcl7p on Gen $4 p$ degradation was addressed to decipher its role in Gen $4 p$ stability regulation in amino acid starved cells. 


\section{Materials and Methods}

\subsection{Materials}

\subsubsection{Chemicals, enzymes, and antibodies}

Chemicals used for the production of buffers, solutions, gels, and culture media were sourced from CARL Roth GmbH \& Co KG (Karlsruhe, D), FluKa (Neu-Ulm, D), GibCo BrL LifE Technologies GmbH (Karlsruhe, D), Invitrogen GmbH (Karlsruhe, D), Merck (Darmstadt, D), OxOID (Hampshire, UK), Roche GmBH (Mannheim, D), and SIgMAAldrich Chemie GmBH (Steinheim, D).

Restriction enzymes, DNA-modifying enzymes, and polymerases were provided by FERmentas (St. Leon-Rot, D), FinnZymes/New England Biolabs GMBH (Frankfurt am Main, D), and Novagen (Darmstadt, D). As DNA size standards 'Gene Ruler $1 \mathrm{~kb}$ DNA ladder' and 'Gene Ruler 100 bp DNA ladder' from FERMENTAS (St. Leon-Rot, D) were used. Agarose for preparation of gels was provided by CARL RoTH GMBH \& Co KG (Karlsruhe, D). Kits for preparation of plasmid DNA from Escherichia coli and extraction of DNA from gels were obtained from QIAGEN (Hilden, D). Synthetic oligonucleotides were purchased from Eurofins MWG Operon (Ebersberg, D) and InVITROGEN GMBH (Karlsruhe, D).

Bradford solution 'RotiQuant' for the determination of protein concentration was provided by Rотн GмвH \& Co KG (Karlsruhe, D). As markers for determination of protein weight 'PageRuler Prestained Protein Ladder' and 'Prestained Protein Molecular Weight Marker' were used. SDS protein gels were blotted on nitrocellulose membrane obtained from Schleicher \& Schuell Bioscience GmbH (Dassel, D). Antibodies were produced by Molecular Probes (Eugene, OR USA) and Santa Cruz Biotechnology Inc. (Santa Cruz, CA, USA) and detected on Hyperfilm ${ }^{\mathrm{TM}}$ ECLL $^{\mathrm{TM}}$ from AMERSHAM PHARMACIA BIOTECH (Buckinghamshire, UK). 


\subsubsection{Strains}

\subsubsection{Escherichia coli}

For general cloning procedures and plasmid propagation the E. coli strain DH5 $\alpha F^{\prime}$,

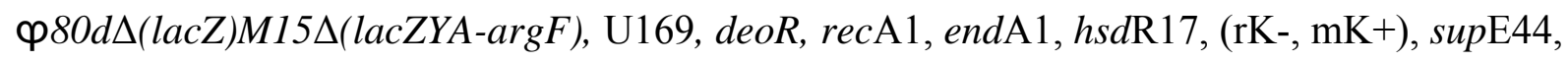
$\lambda$-, thi-1, gyrA96, relA1] (Woodcock et al., 1989) was used.

\subsubsection{Saccharomyces cerevisiae}

All yeast strains used in this study are congenic to W303 genetic background beside the strains Y01443 and Y02797 which are derivatives of the S288c genetic background. Details of the strains are given in Table 1. The yeast strain RH3393 was obtained by replacing the PHO85 genomic locus with a module that confers kanamycin resistance. The PHO85 coding sequence was deleted by homologous recombination using the kanamycin resistance cassette (pho85::kanMX4) of the Euroscarf strain Y02797 (Brachmann et al., 1998) with additional sequences homologous to the 5' upstream and 3' downstream regions of the gene. The PCR product (primers KB84/KB85) was transformed into the $\Delta$ pcl5 strain RH3238 (Bömeke et al., 2006) and plated on rich medium containing $200 \mu \mathrm{g} / \mathrm{ml}$ Geneticin G418 sulfate (GIBCO, UK). Transformants were replica-plated and disruption of PHO85 was confirmed by Southern hybridization using a probe derived from the 5' flanking region of the PHO85 locus amplified from genomic DNA with the primers KB84 and oFLS111.

The yeast strain RH3394 was created by PCR-mediated gene replacement of the genomic PCL7 locus via homologous recombination using the kanamycin resistance cassette. Primers RP141/RP142 were designed specifically for amplification of pcl7::kanMX4 with additional 500 bp upstream and downstream of the resistance cassette from chromosomal DNA of the Euroscarf strain EY1443 (pcl7::kanMX4) (Brachmann et al., 1998). The resulting PCR product was transformed into the pcl5 deletion strain RH3238 and plated on YPD medium supplemented with $200 \mu \mathrm{g} / \mathrm{ml}$ Geneticin G418 sulfate. Transformants were replica-plated and genomic DNA was isolated to verify deletions by Southern hybridization using a 5 ' flanking region probe received by digestion of the deletion cassette with BamHI. 
Table 1: Saccharomyces cerevisiae strains used in this study

\begin{tabular}{|c|c|c|}
\hline Yeast strain & Genotype & Source \\
\hline RH2704 & $\begin{array}{l}\text { MAT } \alpha, \text { rsl1-4(kap95ts), ura3, trp1, leu2, } \\
\text { ade2 }\end{array}$ & (Koepp et al., 1996) \\
\hline RH3237 & MATa, ura3-1, can1-100, leu2-3, trp1-1 & (Bömeke et al., 2006) \\
\hline RH3238 & $\begin{array}{l}\text { MATa, ura3-1, can1-100, leu2-3, trp1-1, } \\
\text { pcl5::hisG }\end{array}$ & (Bömeke et al., 2006) \\
\hline RH3239 & $\begin{array}{l}\text { MATa, leu2-3, ura3-52, gal2, PCL5- } \\
\text { yEGFP-kanMX }\end{array}$ & (Bömeke, 2006) \\
\hline RH3241 & $\begin{array}{l}\text { MATa, ade2, trp1, leu2-3, his3, ura3-52, } \\
\text { pho81::HIS3 }\end{array}$ & (Ogawa et al., 1995) \\
\hline RH3393 & $\begin{array}{l}\text { MATa, ura3-1, can1-100, leu2-3, trp1-1, } \\
\text { pcl5::hisG, pho85::kanMX4 }\end{array}$ & This study \\
\hline RH3394 & $\begin{array}{l}\text { MATa, ura3-1, can1-100, leu2-3, trp1-1, } \\
\text { pcl5::hisG, pcl7::kanMX4 }\end{array}$ & This study \\
\hline Y01443 & $\begin{array}{l}\text { BY4741, MATa, his3-1, leu2, met15, ura3, } \\
\text { pcl7::kanMX4 }\end{array}$ & $\begin{array}{l}\text { (Brachmann et al., } \\
\text { 1998) }\end{array}$ \\
\hline Y02797 & $\begin{array}{l}\text { BY4741, MATa, his3-1, leu2, met15, ura3, } \\
\text { pho85::kanMX4 }\end{array}$ & $\begin{array}{l}\text { (Brachmann et al., } \\
\text { 1998) }\end{array}$ \\
\hline
\end{tabular}

\subsubsection{Plasmids and oligonucleotides}

The plasmids used in this study are listed in Table 2. Plasmid construction is described below and sequences of oligonucleotides used in the present study are given in Table 3.

Table 2: Plasmids used in this study

\begin{tabular}{|l|l|l|}
\hline \multicolumn{1}{|c|}{ Plasmid } & \multicolumn{1}{|c|}{ Description } & \multicolumn{1}{c|}{ Source or reference } \\
\hline pBKSII $^{\circledR}$ & $2.96{\mathrm{~kb} \text { vector, } A m p^{R}(\text { bla }), \text { lacZ, ori }}$ & $\begin{array}{l}\text { STRATAGENE (La Jolla, } \\
\text { CA) }\end{array}$ \\
\hline pRS414 & TRP1, CEN, Amp ${ }^{R}($ bla $)$, ori & $\begin{array}{l}\text { (Sikorski and Hieter, } \\
1989)\end{array}$ \\
\hline
\end{tabular}




\begin{tabular}{|c|c|c|}
\hline Plasmid & Description & Source or reference \\
\hline pRS415 & LEU2, CEN, Amp ${ }^{R}$ (bla), ori & $\begin{array}{l}\text { (Sikorski and Hieter, } \\
\text { 1989) }\end{array}$ \\
\hline pRS416 & URA3, CEN, Amp ${ }^{R}$ (bla), ori & $\begin{array}{l}\text { (Sikorski and Hieter, } \\
\text { 1989) }\end{array}$ \\
\hline pRS425 & LEU2, $2 \mu m, A_{m p}^{R}$ (bla), ori & $\begin{array}{l}\text { (Sikorski and Hieter, } \\
\text { 1989) }\end{array}$ \\
\hline pRS426 & URA3, $2 \mu m, A_{m p}^{R}$ (bla), ori & $\begin{array}{l}\text { (Sikorski and Hieter, } \\
\text { 1989) }\end{array}$ \\
\hline p414GAL1 & $\begin{array}{l}\text { pRS414 containing GAL1 promoter, CYC1 } \\
\text { terminator }\end{array}$ & (Mumberg et al., 1994) \\
\hline p415GAL1 & $\begin{array}{l}\text { pRS415 containing GAL1 promoter, CYC1 } \\
\text { terminator }\end{array}$ & (Mumberg et al., 1994) \\
\hline p425GAL1 & $\begin{array}{l}\text { pRS } 425 \text { containing GAL1 promoter, } C Y C 1 \\
\text { terminator }\end{array}$ & (Mumberg et al., 1994) \\
\hline p416MET25 & $\begin{array}{l}\text { pRS416 containing MET25 promoter, CYC1 } \\
\text { terminator }\end{array}$ & (Mumberg et al., 1994) \\
\hline p425MET25 & $\begin{array}{l}\text { pRS425 containing MET25 promoter, CYC1 } \\
\text { terminator }\end{array}$ & (Mumberg et al., 1994) \\
\hline p426MET25 & $\begin{array}{l}\text { pRS426 containing MET25 promoter, } C Y C 1 \\
\text { terminator }\end{array}$ & (Mumberg et al., 1994) \\
\hline pYGEX-2T & GAL1-10prom-GST, URA3, $2 \mu \mathrm{m}$ & $\begin{array}{l}\text { (Schlenstedt et al., } \\
\text { 1995) }\end{array}$ \\
\hline pME2228 & MET25prom-GFP-PHO81 fusion in p426MET25 & (Bömeke et al., 2006) \\
\hline pME2230 & MET25prom-GFP-PCL7 fusion in p426MET25 & (Bömeke et al., 2006) \\
\hline pME2536 & MET25prom-Strep in p426MET25 & our collection \\
\hline pME2846 & MET25prom-PCL5-GFP-fusion in p426MET25 & (Bömeke, 2006) \\
\hline pME2848 & GAL1prom-myc ${ }^{3}$-GCN4-fusion in p415GAL1 & (Bömeke, 2006) \\
\hline pME2849 & MET25prom-GFP-fusion in p426MET25 & (Bömeke, 2006) \\
\hline pME2853 & $\begin{array}{l}\text { MET25prom-PCL5 }{ }_{a a 1-180} \text { GFP-fusion in } \\
\text { p426MET25 }\end{array}$ & (Bömeke, 2006) \\
\hline pME2854 & $\begin{array}{l}\text { MET25prom-PCL5 }{ }_{a a 61-229} \text { GFP-fusion in } \\
\text { p426MET25 }\end{array}$ & (Bömeke, 2006) \\
\hline
\end{tabular}




\begin{tabular}{|c|c|c|}
\hline Plasmid & Description & Source or reference \\
\hline pME2858 & $\begin{array}{l}\text { MET25prom-PCL5 } 5_{a a 61-180-G F P} \text { fusion in } \\
\text { p426MET25 }\end{array}$ & (Bömeke, 2006) \\
\hline pME2859 & $\begin{array}{l}\text { MET25prom-PCL5 aa181-229-GFP fusion in } \\
\text { p426MET25 }\end{array}$ & (Bömeke, 2006) \\
\hline pME2865 & GAL1-10prom-myc ${ }^{9}-P C L 5$ fusion in $\mathrm{p} 425 \mathrm{GAL} 1$ & (Bömeke et al., 2006) \\
\hline pME2866 & GAL1-10prom-GST-PHO85 fusion in pYGEX-2T & (Bömeke et al., 2006) \\
\hline pME2860 & 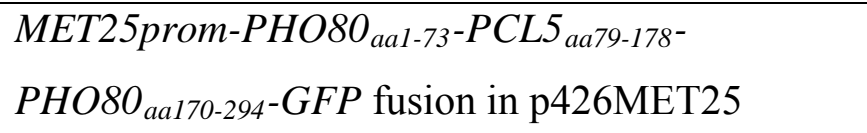 & (Bömeke, 2006) \\
\hline pME2933 & MET25prom-PCL7 in p424MET25 & (Bömeke et al., 2006) \\
\hline pME2948 & $\begin{array}{l}\text { MET25prom-PHO80 aa1-73-PCL5 } 5_{a a 79-178-G F P} \\
\text { fusion in p426MET25 }\end{array}$ & (Bömeke, 2006) \\
\hline pME3370 & $\begin{array}{l}\text { MET25prom-PCL5 } a a 61-229 * * *-G F P \text { fusion } \\
\text { containing three Pc15p amino acid substitutions } \\
\text { K209A, R210A, and R212A in p426MET25 }\end{array}$ & This study \\
\hline pME3371 & $\begin{array}{l}\text { MET25prom-PCL5 aa61-180-NLSSV40-GFP fusion } \\
\text { in p426MET25 }\end{array}$ & This study \\
\hline pME3372 & GAL1-10prom-GST-KAP95 fusion in pYGEX-2T & This study \\
\hline pME3431 & $\begin{array}{l}\text { MET25prom-STREP-PHO85 fusion in } \\
\text { p426MET25 }\end{array}$ & This study \\
\hline pME3447 & $\begin{array}{l}\text { GAL1-10prom-GST-KAP104 fusion in pYGEX- } \\
2 \mathrm{~T}\end{array}$ & This study \\
\hline pME3448 & GAL1-10prom-GST-PSE1 fusion in pYGEX-2T & This study \\
\hline pME3572 & GAL1prom-myc ${ }^{3}-G C N 4$ fusion in p414GAL1 & This study \\
\hline pME3573 & $\begin{array}{l}\text { MET25prom-PCL5 }{ }_{a a 1-180}-G F P \text { fusion in } \\
\text { p416MET25 }\end{array}$ & This study \\
\hline pME3574 & $\begin{array}{l}\text { MET25prom-PCL5 }{ }_{a a 79-178-G F P} \text { fusion in } \\
\text { p426MET25 }\end{array}$ & This study \\
\hline pME3575 & PCL5prom-CYC1term in pRS416 & This study \\
\hline pME3576 & $\begin{array}{l}\text { PCL5prom-PHO80 }{ }_{a a 1-73-P C L 5_{a a 79-178}-G F P} \text { fusion } \\
\text { in pME3575 }\end{array}$ & This study \\
\hline
\end{tabular}




\begin{tabular}{|c|c|c|}
\hline Plasmid & Description & Source or reference \\
\hline pME3577 & $\begin{array}{l}\text { MET25prom-PCL5 aa1-229***-GFP containing three } \\
\text { Pc15p amino acid substitutions K209A, R210A, } \\
\text { and R212A in } \mathrm{p} 426 \mathrm{MET} 25\end{array}$ & This study \\
\hline pME3578 & $\begin{array}{l}\text { MET25prom-PCL5 }{ }_{a a 181-229^{* * *}-G F P \text { containing }} \\
\text { three Pc15p amino acid substitutions K209A, } \\
\text { R210A, and R212A in p426MET25 }\end{array}$ & This study \\
\hline pME3579 & $\begin{array}{l}\text { GAL1-10prom-myc }{ }^{9}-P C L 5 * * * \text { fusion containing } \\
\text { three Pc15p amino acid substitutions K209A, } \\
\text { R210A, and R212A in p425GAL1 }\end{array}$ & This study \\
\hline pME3580 & $\begin{array}{l}\text { GAL1-10prom-myc }{ }^{9}-P C L 5_{a a 181-229} \text { fusion in } \\
\text { p425GAL1 }\end{array}$ & This study \\
\hline pME3581 & $\begin{array}{l}\text { GAL1-10prom-myc }{ }^{9}-\text { PCL5 }_{\text {aa181-229*** fusion }} \\
\text { containing three Pcl5p amino acid substitutions } \\
\text { K209A, R210A, and R212A in p425GAL1 }\end{array}$ & This study \\
\hline pME3583 & MET25-KAP95 in p425MET25 & This study \\
\hline pME3721 & $\begin{array}{l}\text { GAL1-10prom-myc }{ }^{9}-\text { PHO80 }_{a a 1-73-P C L 5}{ }_{a a 79-178^{-}} \\
\text {PHO80 }_{\text {aa170-294 }} \text { fusion in } \mathrm{p} 425 \mathrm{GAL1}\end{array}$ & This study \\
\hline pME3722 & $\begin{array}{l}\text { GAL1-10prom-myc }{ }^{9}-P H O 80_{a a 1-73}-P C L 5_{a a 79-178} \\
\text { fusion in p425GAL1 }\end{array}$ & This study \\
\hline pME3723 & $\begin{array}{l}\text { GAL1-10prom-myc }{ }^{9} \text {-PCL5 }{ }_{\text {aa79-178 }}-\text { PHO80 }_{\text {aa170-294 }} \\
\text { fusion in p425GAL1 }\end{array}$ & This study \\
\hline pME3724 & $\begin{array}{l}\text { GAL1-10prom-myc }{ }^{9} \text {-PCL5 } a a 1-180 \text { fusion in } \\
\text { p425GAL1 }\end{array}$ & This study \\
\hline pME3725 & $\begin{array}{l}\text { GAL1-10prom-myc }{ }^{9}-P C L 5_{a a 61-229} \text { fusion in } \\
\text { p425GAL1 }\end{array}$ & This study \\
\hline pME3726 & $\begin{array}{l}\text { GAL1-10prom-GST-PHO85 }{ }^{E 53 A} \text { fusion in } \\
\text { pYGEX-2T }\end{array}$ & This study \\
\hline pME3727 & MET25prom-myc ${ }^{9}$-PCL5 fusion in p415MET25 & This study \\
\hline pME3728 & GAL1prom-myc ${ }^{3}$-GCN4-fusion in p425GAL1 & This study \\
\hline pME3729 & $\begin{array}{l}\text { PCL5***-ORF containing three Pcl5p amino acid } \\
\text { substitutions K209A, R210A, and R212A in } \\
\text { pBKSII }^{\circledR}\end{array}$ & This study \\
\hline
\end{tabular}




\begin{tabular}{|l|l|l|}
\hline \multicolumn{1}{|c|}{ Plasmid } & \multicolumn{1}{|c|}{ Description } & \multicolumn{1}{|c|}{ Source or reference } \\
\hline KB294 & $\begin{array}{l}\text { GAL1-10prom-myc }{ }^{3}-G C N 4 \text { fusion in URA3 } \\
\text { marked } 2 \mu m \text { vector }\end{array}$ & (Pries et al., 2002) \\
\hline pVW884 & $\begin{array}{l}\text { TETprom-HA }{ }^{2}-P H O 85^{E 53 A} \text { fusion in URA3 } \\
\text { marked } C E N \text { vector }\end{array}$ & (Wanke et al., 2005) \\
\hline
\end{tabular}

\subsubsection{Plasmid construction}

The construction of plasmids pME3370, pME3577, and pME3578 was started by site-directed mutagenesis of the PCL5-ORF via KOD polymerase using the primers oFLS35 and oFLS36 (see Table 3) resulting in amino acid substitutions K209A, R210A, and R212 and was introduced in pBKSII ${ }^{\circledR}$ yielding pME3729. The mutated PCL $5^{* * *}-\mathrm{ORF}(\mathrm{KB} 17 / \mathrm{KB} 18)$ and the mutated fragments PCL5 aa61-229*** $(\mathrm{KB} 35 / \mathrm{KB} 18)$ and PCL5 ${ }_{a 181-229^{* * *}}(\mathrm{~KB} 54 / \mathrm{KB} 18)$ were introduced as SpeI/SmaI fragments in front of the GFP-ORF of plasmid pME2849. Plasmid pME3574 encoding the cyclin box domain of Pc15p (aa79-178) fused to GFP was yielded by amplification of the fragment and insertion as SpeI/SmaI fragment into pME2849.

To obtain pME3371, the Pc15aa61-180 encoding fragment was amplified with Pfu polymerase using the primers KB35 and KB100 and ligated as SpeI/SmaI fragment in front of the GFP-ORF of pME2849. Plasmid pME3573 was constructed by amplification of the Pcl5aa1-180-GFP encoding fragment from plasmid pME2853 with the oligonucleotides KB17 and RP72 and ligation as SpeI/ClaI fragment into p416MET25.

The plasmid pME3576 was constructed in two steps. First, the PCL5 promoter (coordinates -890 to -3) was amplified as $\mathrm{SacI} / \mathrm{XbaI}$ fragment using the primers oFLS95 and oFLS96 and ligated in SacI/XbaI-digested p416MET25, leading to pME3575. Next, the hybrid ORF PHO80 $_{b p 1-219}-P C L 5_{b p 235-534-G F P}$ was amplified with the oligonucleotides oFLS97 and oFLS98 from plasmid pME2948 and inserted as SpeI/ClaI fragment into XbaI/ClaI-digested pME3575.

To construct a fusion glutathione $S$-transferase (GST) with full-length Kap95p, the KAP95 coding region was amplified by PCR using Pfu polymerase and the primers Kap95-BamHISTART and Kap95-STOP-XbaI and inserted as a BamHI/XbaI fragment in-frame downstream of the GST-ORF into pYGEX-2T to yield pME3372. To obtain plasmid pME3447, the KAP104-ORF was amplified by PCR using the primers oFLS74 and oFLS75 
and inserted as BamHI/SpeI fragment in-frame downstream of the GST-ORF of pYGEX-2T. Plasmid pME3448 used for expression of GST-PSE1 was similarly constructed using the oligonucleotides oFLS76 and oFLS77. To receive plasmid pME3579 expressing a nine-fold myc epitope-tagged fusion of the NLS-mutated PCL5***-ORF (containing the amino acid substitutions K209A, R210A, and R212A), the mutated PCL5***-ORF was amplified with Pfu polymerase using primers KB7/RP125 and inserted as a SmaI/HindIII fragment into p425GAL1. A 360-bp BglII fragment carrying myc $^{9}$ was introduced into a BglII site in front of the third amino acid of Pcl5***. Similarly, plasmids pME3580 encoding myc ${ }^{9}-$ Pcl5aa181-229 (oFLS104/RP125) and pME3581 encoding myc ${ }^{9}-$ Pcl5aa181-229*** with mutated NLS motif were constructed. After amplification of the corresponding fragment with Pfu polymerase and insertion as a SmaI/HindIII fragment into p425GAL1 a 360-bp BglII fragment encoding $\mathrm{myc}^{9}$ was introduced after the first amino acid of the respective Pcl5p fragment. Plasmid construction of pME3721 was achieved by amplification of $\mathrm{PHO} \mathrm{O}_{\text {aa1-73- }}$ PCL5 aa79-178-PHO80 $_{a a 170-294}$ with the primers oFLS90 and oFLS87 using pME2860 as template. The fragment was inserted as SmaI/XhoI fragment into p425GAL1 and the nine-fold myc epitope encoding BglII fragment was subsequently introduced in a BglII restriction site after the start codon. The plasmids pME3722 encoding myc ${ }^{9}-P H O 80_{a a 1-73}-P C L 5_{a a 79-178}$ and pME3723 encoding myc ${ }^{9}-$ PCL5 $_{a a 79-178-P H O 80_{a a 170-294}}$ were similarly obtained using oligonucleotides oFLS90/oFLS94 and oFLS92/oFLS87, respectively. Plasmids pME3724 and pME3725 were constructed in an analogous way. The myc $^{9}-P C L 5_{\text {aa1-180 }}$ fragment encoded on plasmid pME3724 was amplified without $m y c^{9}$ fragment using primers KB7 and oFLS114 and introduced as SmaI/HindIII fragment into p425GAL1 before the nine-fold myc epitope was introduced behind the first amino acid as a BglII fragment. Similarly, pME3725 encoding myc ${ }^{9}$-Pc15aa61-229 was obtained using primers oFLS116 and RP125 for amplification.

To express GST-PHO85 ${ }^{E 53 A}$ under control of the GAL1 promoter, the mutated PHO85 ${ }^{E 53 A}$-ORF was amplified from plasmid pVW884 with the primers KB31/KB32 and inserted as BamHI/SpeI fragment into pYGEX-2T leading to pME3726.

For expression of KAP95, the ORF was amplified as a BamHI/SalI fragment and introduced into BamHI/SalI-digested p425MET25 to yield pME3583. To express PHO85, the coding region on plasmid pME2866 was amplified using the oligonucleotides oFLS4 and oFLS5 and the fragment was inserted as BamHI/ClaI fragment into pME2536.

Construction of plasmid pME3727 was achieved by insertion of the myc ${ }^{9}$-PCL5 encoding region from plasmid pME2865 into p415MET25 as SmaI/HindIII fragment. 
Plasmid pME3728, expressing a triple myc epitope-tagged version of GCN4 under control of the GAL1 promoter, was obtained by amplifying GCN4 with Pfu polymerase and oligonucleotides RP89 and BH149 and subsequent insertion in p425GAL1 as blunt/HindIII fragment. A 120-bp BamHI fragment carrying the triple myc epitope was inserted into a BglII restriction site corresponding to a position after the fifth amino acid of Gen $4 p$. To construct plasmid pME3572, the triple myc epitope-tagged version of GCN4 was amplified with Pfu polymerase from plasmid pME2848 using the primers oFLS34 and oFLS15 and inserted as a SpeI/ClaI fragment into p414GAL1.

Table 3: Primers used in this study

\begin{tabular}{|c|c|c|}
\hline Primer & Sequence 5'- 3' & Length \\
\hline oFLS4 & $\begin{array}{l}\text { CGC GGA TCC GCG ATG TCT TCT TCT TCA CAG TAT GTA } \\
\text { G }\end{array}$ & $37 \mathrm{mer}$ \\
\hline oFLS5 & CCA TCG ATG GTT ATG AAG CGT GGT GGT AG & $29 \mathrm{mer}$ \\
\hline oFLS15 & GGG ACC TAG ACT TCA GGT TGT CTA ACT CC & $29 \mathrm{mer}$ \\
\hline oFLS34 & CCT TCG TGT AAT ACA GGG TCG & $21 \mathrm{mer}$ \\
\hline oFLS35 & $\begin{array}{l}\text { GCA TCA TAA TCA TTG TCT GAT TCT GCG GGA GCG GCC } \\
\text { ACC GGA GCG TTG GCG GGA GTT CCG }\end{array}$ & $60 \mathrm{mer}$ \\
\hline oFLS36 & $\begin{array}{l}\text { CGG AAC TCC CGC CAA CGC TCC GGT GGC CGC TCC CGC } \\
\text { AGA ATC AGA CAA TGA TTA TGA TGC }\end{array}$ & $60 \mathrm{mer}$ \\
\hline oFLS74 & CGC GGA TCC GCG ATG GCA TCG ACA TGG AAG CCC G & $34 \mathrm{mer}$ \\
\hline oFLS75 & GGA CTA GTC CTT AGC TGG TGA ACT GTT GTA AGA AG & $35 \mathrm{mer}$ \\
\hline oFLS76 & CGC GGA TCC GCG ATG TCT GCT TTA CCG GAA GAA G & $34 \mathrm{mer}$ \\
\hline oFLS77 & GGA CTA GTC CTT ATG CAA ACC ATT TAT GTA CTT TC & $34 \mathrm{mer}$ \\
\hline oFLS87 & $\begin{array}{l}\text { CCG CTC GAG TTA ATC TGG CTT TGA TCG CTT GTT ATA } \\
\text { G }\end{array}$ & $36 \mathrm{mer}$ \\
\hline oFLS90 & $\begin{array}{l}\text { TCC CCC GGG ATG AGA TCT GAA AGC ACA TCA GGA } \\
\text { GAA CGT TC }\end{array}$ & $41 \mathrm{mer}$ \\
\hline oFLS92 & $\begin{array}{l}\text { TCC CCC GGG ATG AGA TCT AAC ATT TTA AAA TTT CTA } \\
\text { AAT GAA GTG }\end{array}$ & $45 \mathrm{mer}$ \\
\hline oFLS94 & CCG CTC GAG TTA GAG CTC ATA ATT CAG CTT ACC C & $34 \mathrm{mer}$ \\
\hline oFLS95 & CGT GAG CTC CGT GGC ATC TTT GCA GCA CAC & $30 \mathrm{mer}$ \\
\hline
\end{tabular}




\begin{tabular}{|c|c|c|}
\hline Primer & Sequence 5'- 3' & Length \\
\hline oFLS96 & $\begin{array}{l}\text { GGG TCT AGA TTT ATT TTT ATT TTT CTT TTT TTT TTT } \\
\text { TTT TTT TTT TTT TTT TTT AAA AAC }\end{array}$ & $66 \mathrm{mer}$ \\
\hline oFLS97 & CGC ACT AGT ATG GAA AGC ACA TCA GGA GAA C & $31 \mathrm{mer}$ \\
\hline oFLS98 & GCC ATC GAT TTA TTT GTA GAG CTC ATC CAT GC & $30 \mathrm{mer}$ \\
\hline oFLS104 & $\begin{array}{l}\text { TCC CCC GGG ATG AGA TCT CCA TAT GAC GAG TTT TTG } \\
\text { CTA TG }\end{array}$ & $41 \mathrm{mer}$ \\
\hline oFLS111 & GGT ATT GCT CAA GTT TGC CCA GTT TG & $35 \mathrm{mer}$ \\
\hline oFLS114 & TCC AAG CTT TTA AAT GGC CAG CTC ATA ATT CAG & $42 \mathrm{mer}$ \\
\hline oFLS116 & $\begin{array}{l}\text { TCC CCC GGG ATG AGA TCT TTG TCC GAG ATC AGT CGG } \\
\text { СCT C }\end{array}$ & $40 \mathrm{mer}$ \\
\hline $\begin{array}{l}\text { Kap95- } \\
\text { BamHI- } \\
\text { START }\end{array}$ & CGC GGA TCC GCG ATG TCC ACC GCT GAA TTT GC & $32 \mathrm{mer}$ \\
\hline $\begin{array}{l}\text { Kap95- } \\
\text { XbaI-STOP }\end{array}$ & GCT CTA GAG CTT ATA AGG ATA ATT GAC GCT TC & $32 \mathrm{mer}$ \\
\hline KB7 & $\begin{array}{l}\text { TCC CCC GGG ATG GAT GGA AGA TCT AAT CAT AGG TTT } \\
\text { ACT CCA GAT TCC }\end{array}$ & $48 \mathrm{mer}$ \\
\hline KB17 & GGA CTA GTC CAT GGA TGG AAA TCA TAG GTT T & $31 \mathrm{mer}$ \\
\hline KB18 & TCC CCC GGG GGA ACA AGA TTT AAT CTG TTT CCA & $33 \mathrm{mer}$ \\
\hline KB31 & CGC GGA TCC GCG ATG TCT TCT TCT TCA CAG TAT GTA & $36 \mathrm{mer}$ \\
\hline KB32 & GGA CTA GTC CTT ATG AAG CGT GGT GGT AGT A & $31 \mathrm{mer}$ \\
\hline KB35 & GGA CTA GTC CAT GTT GTC CGA GAT CAG TCG GCC T & $34 \mathrm{mer}$ \\
\hline KB54 & GGA CTA GTC CAT GCC ATA TGA CGA GTT TTT G & $31 \mathrm{mer}$ \\
\hline KB84 & $\begin{array}{l}\text { GAG AAA GGA GAA GTA TCA CAA GAT AGC AAT ATA } \\
\text { ATC GG }\end{array}$ & $38 \mathrm{mer}$ \\
\hline KB85 & $\begin{array}{l}\text { GCT GGG TGG CTA CGA ATA AGA AGG CCA AGA TTA } \\
\text { GAA GTG TG }\end{array}$ & $41 \mathrm{mer}$ \\
\hline KB100 & $\begin{array}{l}\text { TCC CCC GGG GGA AAC TTT ACG TTT CTT TTT GGG AAT } \\
\text { GGC CAG CTC ATA ATT CAG }\end{array}$ & $54 \mathrm{mer}$ \\
\hline RP72 & CCA TCG ATT TTG TAG AGC TCA TAA ATG CCA TG & $32 \mathrm{mer}$ \\
\hline RP89 & $\begin{array}{l}\text { TAA ATG TCC GAA TAT GGA AGA TCT TCC CAG CCA AGT } \\
\text { TTA TTT GCT TTA AAT }\end{array}$ & $51 \mathrm{mer}$ \\
\hline
\end{tabular}




\begin{tabular}{|l|l|c|}
\hline \multicolumn{1}{|c|}{ Primer } & \multicolumn{1}{|c|}{ Sequence 5'-3, } & Length \\
\hline RP125 & CCC AAG CTT TTA ACA AGA TTT AAT CTG TTT CCA AG & 35mer \\
\hline RP141 & $\begin{array}{l}\text { ATA CGG TGT GTT TTT CGC TGG ATG AGC GAC AGA GGC } \\
\text { GGA }\end{array}$ & $39 \mathrm{mer}$ \\
\hline RP142 & $\begin{array}{l}\text { AGA AAT TCT GGT AGA CAA CTT TTT GCT ATG AAA AGT } \\
\text { GAG }\end{array}$ & $39 \mathrm{mer}$ \\
\hline
\end{tabular}

\subsection{Methods}

\subsubsection{Cultivation of microorganisms}

\subsubsection{Cultivation of Escherichia coli}

Cells were grown in Luria-Bertani (LB) medium (1\% (w/v) Bacto-tryptone, $0.5 \%$ (w/v) yeast extract, $1 \%(\mathrm{w} / \mathrm{v}) \mathrm{NaCl})$ at $37^{\circ} \mathrm{C}$. For selective media ampicillin was added to the medium to a final concentration of $100 \mu \mathrm{g} / \mathrm{ml}$. Solid medium contained $2 \%(\mathrm{w} / \mathrm{v})$ agar.

\subsubsection{Cultivation of Saccharomyces cerevisiae}

The yeast strains were grown in YPD medium $(1 \%(\mathrm{w} / \mathrm{v})$ yeast extract, $1 \%(\mathrm{w} / \mathrm{v})$ peptone, $2 \%$ $(\mathrm{w} / \mathrm{v})$ glucose $)$ or in YNB medium $(0.15 \%(\mathrm{w} / \mathrm{v})$ yeast nitrogen base lacking amino acids and ammonium sulfate, $0.5 \%(\mathrm{w} / \mathrm{v})$ ammonium sulfate, $2 \%(\mathrm{w} / \mathrm{v})$ glucose, galactose or raffinose and supplemented with necessary nutrients as described (Guthrie and Fink, 1991). Solid media contained $2 \%(\mathrm{w} / \mathrm{v})$ agar. Cells were grown at $30^{\circ} \mathrm{C}$ or at $20^{\circ} \mathrm{C}$ in case of temperature sensitive mutants.2.2.2. Preparation and characterization of DNA

\subsubsection{Quick boiling plasmid DNA preparation from E. coli ('STET prep') (Holmes and Quigley, 1981)}

E. coli overnight cultures were harvested by centrifugation $(13,000 \mathrm{rpm}, 1 \mathrm{~min})$ and resuspended in $400 \mu \mathrm{l}$ STET buffer $(8 \%(\mathrm{w} / \mathrm{v})$ sucrose, $0.5 \%(\mathrm{v} / \mathrm{v})$ Triton X-100, $50 \mathrm{mM}$ 
EDTA (pH 8.0), $10 \mathrm{mM}$ Tris-HCl ( $\mathrm{pH} 8.0)$ ). Lysis was triggered by addition of $25 \mu 1$ lysozyme solution $(12 \mathrm{mg} / \mathrm{ml})$ for $5 \mathrm{~min}$ and followed by 40 seconds incubation in a boiling water bath. Cell fragments were sedimented by centrifugation $(13,000 \mathrm{rpm}, 15 \mathrm{~min})$ at $4{ }^{\circ} \mathrm{C}$. The sticky pellet was removed by a sterile toothpick. Plasmid DNA was precipitated by addition of $50 \mu \mathrm{l}$ of $3 \mathrm{M}$ sodium acetate $(\mathrm{pH} 4.8)$ and $500 \mu \mathrm{l}$ isopropanol. After centrifugation $\left(13,000 \mathrm{rpm}, 15 \mathrm{~min}, 4^{\circ} \mathrm{C}\right)$ the DNA was washed with $70 \%$ ethanol and air-dried before it was dissolved in $50 \mu 1$ sterile water or TE buffer (10 mM Tris-HCl (pH 8.0), $1 \mathrm{mM}$ EDTA) and stored at $-20^{\circ} \mathrm{C}$.

\subsubsection{2. 'QIAprep spin miniprep' plasmid isolation from E. coli (QIAGEN, Hilden, D)}

$5 \mathrm{ml}$ of an overnight culture of $E$. coli was harvested by centrifugation (13,000 rpm, 1min) and plasmid DNA preparation was carried out to supplier's specifications.

\subsubsection{Quick DNA preparation from yeast ('Smash \& Grab')}

A $10 \mathrm{ml}$ overnight yeast culture was harvested by centrifugation $(3,000 \mathrm{rpm}, 3 \mathrm{~min})$. After removal of the supernatant, cells were resuspended in $0.5 \mathrm{ml}$ sterile water, transferred to a 1.5 $\mathrm{ml}$ reaction tube, and collected again by centrifugation $(13,000 \mathrm{rpm}, 1 \mathrm{~min})$. The supernatant was discarded and the cells resuspended in the residual liquid. Then, $200 \mu 1$ lysis buffer $(2 \%$ (v/v) Triton X-100, 1\% (w/v) SDS, $100 \mathrm{mM} \mathrm{NaCl,} 10 \mathrm{mM}$ Tris, $1 \mathrm{mM}$ EDTA (pH 8.0)) together with $0.2 \mathrm{ml}$ phenol/ $\mathrm{MeCl}_{2} /$ Tris-EDTA and $200 \mu \mathrm{g}$ of $0.45 \mathrm{~mm}$ glass beads were added and shaken for $10 \mathrm{~min}$ at $4^{\circ} \mathrm{C}$. In addition, $0.2 \mathrm{ml} \mathrm{TE}$ buffer were added to the tube and spun again at maximum speed. The aqueous layer was transferred to a fresh microfuge tube to

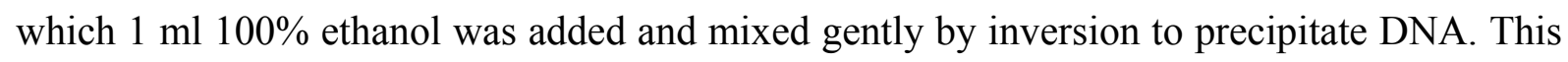
was further centrifuged at maximum speed for $5 \mathrm{~min}$ at room temperature to pellet DNA which was solved in $400 \mu \mathrm{l}$ Tris-EDTA and $3 \mu \mathrm{l}$ RNase A $(10 \mathrm{mg} / \mathrm{ml})$. After $10 \mathrm{~min}$ of incubation at $37^{\circ} \mathrm{C} 10 \mu \mathrm{l}$ of $4 \mathrm{M}$ ammonium acetate and $1 \mathrm{ml} 100 \%$ ethanol was added and mixed by inversion again. This was centrifuged at maximum speed for $3 \mathrm{~min}$ and the supernatant was discarded. The transparent pellet was allowed to dry at room temperature and was finally dissolved in $100 \mu \mathrm{l}$ Tris-EDTA. The DNA was stored at $-20^{\circ} \mathrm{C}$. 


\subsubsection{Determination of DNA concentration}

The concentration of DNA was determined spectrophotometrically using a NanoDrop 'ND1000' from NANODrop TEChNOLOGIES INC. (Wilmington, DE, USA) according to the instruction manual provided.

\subsubsection{DNA sequencing and sequence analysis}

Crucial cloning steps were validated by sequencing on an ABI Prism 310 capillary sequencer (ApPlera Deutschland GMBH, Deutsch) at the Göttingen Genomics Laboratory. Sequences were analysed using the Lasergene software (DNASTAR INC., Madison, WI, USA) or 4Peaks software (http://mekentosj.com/science/4peaks/).

\subsubsection{Cloning techniques}

\subsubsection{Polymerase chain reaction ('PCR') (Saiki et al., 1985)}

Polymerase chain reactions for rapid amplification of DNA were carried out using the thermo-stable enzymes Taq polymerase, Pfu polymerase, KOD-Hifi-DNA polymerase or Phusion ${ }^{\circledR}$ and the thermocycler 'Primus' or 'Primus $96^{\text {plus, }}$ (MWG-BIOTECH, Ebersberg, D). In general, 5-50 pmol of each primer and 10-100 ng DNA in a volume of 20-50 $\mu 1$ were used according to manufacturer's protocol. In case of colony-PCR an E. coli or an S. cerevisiae colony were directly used as template. The temperature/time profile was chosen dependent on the DNA polymerase, the oligonucleotides, and the template size. Synthetic oligonucleotides were purchased from EUROFINS MWG OPERON (Ebersberg, D) and INVITROGEN GMBH (Karlsruhe, D).

\subsubsection{Site-directed mutagenesis}

Site-directed mutagenesis was done to exchange codons in PCL5 using the 'QuickChange II Site-directed Mutagenesis' Kit (Stratagene, La Jolla, CA, USA). The primer design and reaction conditions were according to the instructions provided in the manual. Briefly, two complementary primers containing the desired mutations were designed spanning the target 
region of PCL5. $10 \mathrm{ng}$ of the template was used in the reaction and $125 \mathrm{ng}$ of each primer per reaction were added beside the components of the kit as described in the manual. The annealing temperature used was $55^{\circ} \mathrm{C}$ and the number of cycles was 16 . To digest the parental methylated DNA, $1 \mu \mathrm{l}$ of the enzyme DpnI was added and incubated for 1 hour at $37^{\circ} \mathrm{C}$ and $1 \mu 1$ of this reaction was used for transformation of $E$. coli cells. Plasmids were isolated from transformants and sequenced to verify the presence of the desired mutation.

\subsubsection{DNA restriction}

For analytical purposes, generally 200-500 ng of plasmid DNA was digested at the recommended temperature for 1-2 hours in a total volume of $20 \mu \mathrm{l}$ by 1-2 units restriction enzyme in appropriate buffer (FERMENTAS, ST. LEON-Rot, D, NEW ENGLAND BIOLABS GMBH, FRANKFURT AM MAIN, D). For cloning, preparative restrictions required, dependent on the strategy, more DNA.

\subsubsection{Dephosphorylation of DNA}

For cloning, the restricted vector DNA was dephosphorylated in order to remove 5' phosphates to avoid re-ligation. Therefore, linearized DNA fragments were incubated with 1$2 \mu 1$ shrimp alkaline phosphatase (FERMENTAS, ST. LEON-ROT, D) which was usually added directly to $20 \mu \mathrm{l}$ of the restriction digestion reaction at $37^{\circ} \mathrm{C}$ for 1 hour. The reaction was stopped by incubation at $65^{\circ} \mathrm{C}$ for $10 \mathrm{~min}$.

\subsubsection{Ligation of DNA fragments (Maniatis et al., 1989)}

Linear DNA fragments were mixed in ligation buffer $(20 \mathrm{mM}$ Tris- $\mathrm{HCl}, 10 \mathrm{mM} \mathrm{MgCl}$, 10 mM DTT, 0.6 mM ATP, pH7.6) with 5 units T4-DNA-ligase (FERMENTAS, ST. LEONRot, D) in a total reaction volume of $20 \mu \mathrm{l}$ and incubated overnight at $16^{\circ} \mathrm{C}$ or for 2 hours at room temperature. Molar proportions between vector and insert were chosen approximately at 1:5 to $1: 10$. DNA was used for transformation without further purification. 


\subsubsection{Agarose gel electrophoresis}

DNA solutions were mixed with 0.1 volume 10x DNA sample buffer (25\% (v/v) Ficoll 4000, $0.25 \%(\mathrm{w} / \mathrm{v})$ bromphenolblue, $0.25 \%(\mathrm{w} / \mathrm{v})$ xylene cyanol, $200 \mathrm{mM}$ EDTA ( $\mathrm{pH} 8.0)$ ). DNA fragments were separated in a horizontal Agarose gel containing 1\% Agarose (CARL ROTH GMBH \&Co KG, Karlsuhe, D) in TAE buffer (40 mM Tris-acetate, $20 \mathrm{mM}$ sodium acetate, 2 mM EDTA ( $\mathrm{pH} 8.0)$ ) in presence of $0.5 \mu \mathrm{g} / \mathrm{ml}$ ethidium bromide applying a maximum voltage of 90 volts. DNA bands were detected on a UV-transilluminator (BIORAD, München, D) at a wavelength of $\lambda=254 \mathrm{~nm}$. As DNA size standards 'Gene Ruler $1 \mathrm{~kb}$ DNA ladder' and 'Gene Ruler 100 bp DNA ladder' from FERMENTAS (St. Leon-Rot, D) were used.

\subsubsection{Isolation of DNA fragments}

The desired DNA band was excised from agarose gel. DNA was extracted from gel using 'QIAquick gel extraction kit' (QIAGEN, Hilden, D). Isolation was performed according to the manufacturer's specifications. The purified DNA was solved in sterile water and directly used or stored at $-20^{\circ} \mathrm{C}$.

\subsubsection{Transformation methods}

\subsubsection{Preparation of chemically competent $E$. coli cells (Inoue et al., 1990)}

$250 \mathrm{ml} \mathrm{SOB}$ medium (2\% (w/v) tryptone, $0.5 \%(\mathrm{w} / \mathrm{v})$ yeast extract, $10 \mathrm{mM} \mathrm{NaCl}, 2.5 \mathrm{mM}$ $\mathrm{KCl}, 10 \mathrm{mM} \mathrm{MgCl}_{2}, 10 \mathrm{mM} \mathrm{MgSO}_{4}$ ) were inoculated with E. coli DH5 $\alpha$ in 11 Erlenmeyer flask. The culture was incubated at $20^{\circ} \mathrm{C}$ with gently shaking for at least 24 hours until an $\mathrm{OD}_{600 \mathrm{~nm}}$ of approximately 0.6 was reached. Cells were chilled on ice for $10 \mathrm{~min}$ and harvested by centrifugation $\left(2,500 \mathrm{xg}, 10 \mathrm{~min}, 4^{\circ} \mathrm{C}\right)$. Supernatant was discarded and the cells were resuspended in $80 \mathrm{ml}$ of ice-cold transformation buffer (TB: $10 \mathrm{mM}$ PIPES or $10 \mathrm{mM}$ HEPES, $15 \mathrm{mM} \mathrm{CaCl}_{2}$, and $250 \mathrm{mM} \mathrm{KCl}$; the $\mathrm{pH}$ was then adjusted to 6.7 with $\mathrm{KOH}$ followed by the addition of $55 \mathrm{mM} \mathrm{MnCl}$ ), incubated for $10 \mathrm{~min}$ on ice and centrifuged again $\left(2,500 \mathrm{x} \mathrm{g}, 10 \mathrm{~min}, 4^{\circ} \mathrm{C}\right)$. Then, the cells were carefully resuspended in $20 \mathrm{ml} \mathrm{TB}$ followed by slow addition of DMSO while gently swirling to a final concentration of $7 \%$. 
This cell suspension was again chilled on ice for $10 \mathrm{~min}$ before cells were frozen in aliquots in liquid nitrogen and stored at $-80^{\circ} \mathrm{C}$ until they were used.

\subsubsection{Transformation of $E$. coli (Inoue et al., 1990)}

$200 \mu 1$ of frozen, competent $E$. coli cells were thawed on ice and incubated for 10-30 min with complete ligation reaction or $1 \mu 1$ plasmid DNA to be transformed. The cells were then subjected to heat shock at $42^{\circ} \mathrm{C}$ for $60-90$ seconds and then placed on ice for $3 \mathrm{~min} .800 \mu 1$ SOC medium (SOB $+20 \mathrm{mM}$ glucose) or LB medium were added and the mixture was kept at $37^{\circ} \mathrm{C}$ for 1 hour. The cells were then centrifuged $(2,000 \mathrm{rpm}, 2 \mathrm{~min})$ and plated onto appropriate selective medium.

\subsubsection{Transformation of $S$. cerevisiae by LiOAc method (Ito et al., 1983)}

$10 \mathrm{ml}$ of appropriate medium was inoculated with cells and incubated overnight at $30^{\circ} \mathrm{C}$ on a culture wheel. $400-600 \mu \mathrm{l}$ of this cell suspension were used to inoculate $10 \mathrm{ml}$ medium and incubated at $30^{\circ} \mathrm{C}$ for 5-6 hours. Cells were harvested by centrifugation (3,000 rpm, $\left.3 \mathrm{~min}\right)$. After removal of the supernatant, the cells were washed in 4-5 ml LiOAc/TE (0.1 M LiOAc, $10 \mathrm{mM}$ Tris-HCl (pH 8.0), 1 mM EDTA (pH 8.0)), harvested again (3,000 rpm, $3 \mathrm{~min})$, and finally resuspended in $400 \mu \mathrm{LiOAc} / \mathrm{TE}$.

For each transformation an aliquot of $200 \mu 1$ competent yeast cells was transferred to a sterile reaction tube. $20 \mu \mathrm{l}$ denatured salmon sperm DNA (ssDNA, $10 \mathrm{mg} / \mathrm{ml}$ ), an appropriate amount of DNA for transformation, and $800 \mu \mathrm{l}$ PEG/LiOAc/TE $(50 \% \quad(\mathrm{w} / \mathrm{v})$ PEG (polyethylene glycol) 4000, 0.1 M LiOAc, $2.5 \mathrm{mM}$ Tris-HCl (pH 8.0), $0.25 \mathrm{mM}$ EDTA (pH 8.0)) were added. Each approach was mixed by gently vortexing or inverting the tube, incubated at $30^{\circ} \mathrm{C}\left(20^{\circ} \mathrm{C}\right.$ in case of temperature sensitive mutants) for $30 \mathrm{~min}$, and heatshocked for $20 \mathrm{~min}$ (10-15 $\mathrm{min}$ in case of temperature sensitive mutants) at $42^{\circ} \mathrm{C}$. Cells were harvested by centrifugation (7,000 rpm, 10-20 seconds) and the supernatant was removed. After 1 hour (in case of plasmid-DNA) to up to 3 hours (in case of linear-DNA) of incubation in YPD medium at $30^{\circ} \mathrm{C}$ ( or $20^{\circ} \mathrm{C}$ in case of temperature sensitive mutants), cells were spun down (4,000 rpm, 10-20 seconds), and supernatant was discarded. Cells were resuspended in the remaining liquid and plated out onto appropriate selective solid media. 


\subsubsection{Hybridization techniques}

\subsubsection{Southern hybridization (Southern, 1975).}

For each approach $10 \mu \mathrm{g}$ chromosomal DNA were digested overnight with an appropriate restriction enzyme. DNA fragments were separated in a horizontal Agarose gel containing 1\% Agarose in TAE buffer. After electrophoresis, DNA was transferred to Amersham Hybond ${ }^{\mathrm{TM}}$ N membrane (Ge HealthCARE Life Sciences, München, D) by capillary blotting for at least 4 hours or overnight. DNA was cross-linked by illumination with UV-light $(\lambda=254 \mathrm{~nm})$ for $3 \mathrm{~min}$ from each side and baking at $80^{\circ} \mathrm{C}$ for $30 \mathrm{~min}$. Gel-purified DNA fragments were used as probes. Non-radioactive labelling of probes and detection was performed using the Gene Images $^{\mathrm{TM}}$ Random-Prime DNA labelling kit and the Gene Images ${ }^{\mathrm{TM}}$ CDP-Star ${ }^{\mathrm{TM}}$ Detection Kit (GE Healthcare Life Sciences, München, D) according to the manufacturer's instructions. The chemiluminescent signals were detected using Amersham Hyperfilm ${ }^{\mathrm{TM}} \mathrm{ECL}$ (GE Healthcare Life Sciences, München, D).

\subsubsection{Protein methods}

\subsubsection{Preparation of crude extracts}

$50 \mathrm{ml}$ of culture was harvested at 3,000 rpm for $3 \mathrm{~min}$ and the cell pellet was immediately stored at $-80^{\circ} \mathrm{C}$. For preparation of extracts all steps were performed at $4^{\circ} \mathrm{C}$ or on ice. The cell pellets were thawed on ice and the cells were washed with $2.5 \mathrm{ml}$ b-buffer (100 mM Tris- $\mathrm{HCl}$ (pH 7.5), $200 \mathrm{mM} \mathrm{NaCl}, 20 \%$ (v/v) glycerol, $5 \mathrm{mM}$ EDTA). Cells were harvested again (3,000 rpm, $\left.3 \mathrm{~min}, 4^{\circ} \mathrm{C}\right)$, supernatant was discarded. The cells were resuspended in $500 \mu \mathrm{l}$ b+buffer (b-buffer with freshly added 0.1\% (v/v) $\beta$-mercaptoethanol and 1/25 volume of 'Complete' protease inhibitor mix (Roche, Penzberg, D)) and transferred to a $2 \mathrm{ml}$ reaction tube with $500 \mu \mathrm{l}$ of $0.45 \mathrm{~mm}$ glass beads and shaked with Vortex (Vortex Genie 2, SCIENTIFIC INDUSTRIES INC., New York, NY, USA) at maximum power for 6 minutes at $4^{\circ} \mathrm{C}$. The obtained crude lysate was centrifuged at $13,000 \mathrm{rpm}$ for 12 minutes at $4^{\circ} \mathrm{C}$. The supernatant was collected as total cell lysate. The amount of protein in the supernatant was determined using the Bradford method (see next chapter). An appropriate amount of 3 x SDS loading buffer (0.25 M Tris-HCl (pH 6.8), 15\% (v/v) $\beta$-mercaptoethanol, 30\% (v/v) glycerol, 7\% 
$(\mathrm{w} / \mathrm{v}) \mathrm{SDS}, 0.3 \%(\mathrm{w} / \mathrm{v})$ bromphenolblue) was added to each protein sample, which was finally denatured for $10 \mathrm{~min}$ at $65^{\circ} \mathrm{C}$ and stored at $-20^{\circ} \mathrm{C}$.

\subsubsection{Determination of protein concentration (Bradford, 1976)}

Standards containing 2-20 $\mu \mathrm{g}$ bovine serum albumin (BSA) per $\mathrm{ml}$ were prepared to plot a standard curve. 1:5 diluted Bradford solution RotiQuant (Rотн GMBH \& Co KG (Karlsruhe, D) was used as blank. $10 \mu 1$ of crude extracts were mixed in a cuvette with $990 \mu 1$ of 1:5 diluted Bradford reagent and incubated for $10 \mathrm{~min}$ at room temperature. Absorbance was measured at $\lambda=595 \mathrm{~nm}$ in a UV/Vis spectrophotometer.

\subsubsection{SDS polyacrylamide gel electrophoresis 'SDS-PAGE' (Laemmli, 1970)}

Electrophoretic separation of proteins was carried out in 12\% SDS vertical gels build up on two different gel types: running gel (3.5 ml water, $2.5 \mathrm{ml} 4$ x Lower Tris (1.5 M Tris-Base (pH 8.8), 8 mM EDTA, 0.4\% (w/v) SDS), $4 \mathrm{ml}$ polyacryamide (acrylamide/bisacrylamide (30:0.8)), $25 \mu 1$ APS (ammonium persulfate, 10\% (w/v)), $15 \mu 1$ TEMED (N',N',N',N'-tetraethylen-methylen-diamide) and stacking gel (3.9 ml water, $1.5 \mathrm{ml}$ Upper Tris (0.5 M TrisBase (pH 6.8), 8 mM EDTA, 0.4\% (w/v) SDS), $0.6 \mathrm{ml}$ polyacrylamide (30:0.8), $40 \mu \mathrm{l}$ APS, $20 \mu \mathrm{l}$ TEMED). The gel types were cast and polymerized successively on each other. Volumes of equivalent protein concentrations of the extract were loaded onto the gel. As marker for determination of protein weight 'PageRuler Prestained Protein Ladder' (FERMENTAS, St. Leon-Rot, D) was used. Electrophoresis was carried out in running buffer (25 mM Tris-Base, $250 \mathrm{mM}$ glycine, $0.1 \%(\mathrm{w} / \mathrm{v}) \mathrm{SDS}$ ) applying a voltage of $100 \mathrm{~V}$ for 10 $\min$ and $200 \mathrm{~V}$ for further 40 to $50 \mathrm{~min}$.

\subsubsection{Immunoblotting and detection of proteins (Towbin et al., 1979)}

Yeast cell extracts and SDS-PAGE were performed as described. Transfer of separated proteins to nitrocellulose membrane 'Protan' (SCHLEICHER \& SCHUELL Bioscience GMBH, Dassel, D) was carried out at $35 \mathrm{~V}$ overnight or cooled at $100 \mathrm{~V}$ for 1 hour in a 'Mini TransBlot Electrophoretic Cell' (BIO-RAD Industries GMBH, München, D) in transfer buffer (25 mM Tris-Base, $200 \mathrm{mM}$ glycine, 0.02\% (w/v) SDS, 20\% (v/v) methanol). Optional, the 
membrane was stained with Ponceau S solution $(0.2 \%(\mathrm{w} / \mathrm{v})$ Ponceau S, 3\% (v/v) trichloroacetic acid) for $1 \mathrm{~min}$. To remove dye, membrane was washed for $10 \mathrm{~min}$ in PBS (140 mM NaCl, $10 \mathrm{mM} \mathrm{Na-phosphate} \mathrm{(pH} \mathrm{7.5))} \mathrm{with} \mathrm{4-5 \%} \mathrm{non-fat} \mathrm{milk} \mathrm{powder} \mathrm{or} \mathrm{BSA.}$ Blocking of free binding sites on the membrane was done by incubation for 1 hour at room temperature with fresh PBS/milk powder solution. The membrane was probed with a dilution in PBS/milk powder of mouse anti-GFP monoclonal antibody (1:2000, CLONTECH, Heidelberg, D), rabbit polyclonal antiserum against Cdc28 (1:2000, CLONTECH, Heidelberg, D), rabbit anti-GST polyclonal antibody (1:2000, SANTA CRUZ Biotechnology InC., Santa Cruz, CA, USA), or monoclonal mouse anti-Myc '9E10' antibody (1:2000, SANTA CRUZ Biotechnology InC., Santa Cruz, CA, USA) for 2 hours at room temperature or overnight at $4^{\circ} \mathrm{C}$. The membrane was then washed 3 times for $10 \mathrm{~min}$ in PBS and then incubated for 1 hour with 1:5000 dilution of peroxidase-coupled rabbit anti-mouse IgG (DIANOVA GMBH, Hamburg, D), goat anti-mouse IgG or goat anti-rabbit IgG (INVITROGEN GMBH, Karlsruhe, D) antibody in PBS/milk powder. The membrane was again washed 3 times for 10 min with PBS and then the detection of proteins on membrane was carried out using the Enhanced Chemiluminescence (ECL) method (Tesfaigzi et al., 1994) involving two solutions. $10 \mathrm{ml}$ of solution A (2.5 mM Luminol, $40 \mu \mathrm{M}$ paracoumaric acid, $100 \mathrm{mM}$ Tris- $\mathrm{HCl}(\mathrm{pH} \mathrm{8.5))} \mathrm{was}$ mixed with $10 \mathrm{ml}$ solution $\mathrm{B}\left(5.4 \mathrm{mM} \mathrm{H}_{2} \mathrm{O}_{2}, 100 \mathrm{mM}\right.$ Tris- $\left.\mathrm{HCl}(\mathrm{pH} 8.5)\right)$ just prior to use. The membrane was incubated in the reagent while gently shaking for 1 minute and exposed to Amersham Hyperfilm ${ }^{\mathrm{TM}}$ ECL (GE HeAlthCARE LifE SCIENCES, München, D) in the dark until signal intensity was appropriately visible. The film was developed using a 'Optimax' developing machine (Protec Medizintechnik GMBH \& Co KG, Oberstenfeld, D).

\subsubsection{GST pull down assay}

Yeast strains expressing GST as control or GST-fusion proteins together with myc-tagged Pcl5p-variants were pre-grown in selective minimal medium with raffinose as carbon source ( $250 \mathrm{ml}$ or $500 \mathrm{ml}$ if divided into two cultures to apply amino acid starvation) to an $\mathrm{OD}_{600 \mathrm{~nm}}$ of approximately 0.4 . To induce expression of GAL1-driven fusions, $2 \%(\mathrm{v} / \mathrm{v})$ galactose was added. After 3 hours of induction half of the cells were harvested by centrifugation $(3,000$ $\mathrm{rpm}, 4 \mathrm{~min})$ and shifted to minimal medium with $2 \%(\mathrm{v} / \mathrm{v})$ galactose but lacking tryptophan for $45 \mathrm{~min}$ to apply amino acid starvation. Cells were collected by centrifugation (3,000 rpm, $\left.5 \mathrm{~min}, 4^{\circ} \mathrm{C}\right)$ and washed twice with $2.5 \mathrm{ml} \mathrm{B}$-buffer $(50 \mathrm{mM}$ Tris- $\mathrm{HCl}(\mathrm{pH} 7.5), 100 \mathrm{mM}$ $\mathrm{NaCl}, 5 \mathrm{mM}$ EDTA). After centrifugation cells were resuspended in $600 \mu \mathrm{l}$ B+-buffer 
(B-buffer with 1/25 volume of 'Complete' protease inhibitor mix (RocHE, Penzberg, D)) and transferred to a $2 \mathrm{ml}$ reaction tube with $500 \mu 1$ of $0.45 \mathrm{~mm}$ glass beads and vortexed (Vortex Genie 2, Scientific Industries InC., New York, NY, USA) at $4^{\circ} \mathrm{C}$ for 6 min. After breaking up the cells, $300 \mu \mathrm{B}++$-buffer (B+-buffer with $0.08 \%(\mathrm{v} / \mathrm{v})$ Triton X-100) were added to each sample, mixed by inversion, and centrifuged (13,000 rpm, $\left.10 \mathrm{~min}, 4^{\circ} \mathrm{C}\right) .10 \mu \mathrm{l}$ of the extracts were removed to determine total protein concentration. $128 \mu 1$ of the supernatant were transferred to a $1.5 \mathrm{ml}$ reaction tube and denatured by addition of $128 \mu 13 \mathrm{x}$ SDS loading buffer and heating for $10 \mathrm{~min}$ at $65^{\circ} \mathrm{C} .600 \mu \mathrm{l}$ of the remaining extract were mixed with $650 \mu \mathrm{l}$ B++-buffer, $250 \mu 150 \%$ (v/v) glycerol, and $100 \mu \mathrm{l}$ 'washed' GlutathionSepharose ${ }^{\mathrm{TM}}$ 4B-Beads (Ge HealthCARE LifE ScienCES, München, D) ('washing': $1.5 \mathrm{ml}$ beads were washed twice with $10 \mathrm{ml}$ cold PBS (140 mM NaCl, $10 \mathrm{mM}$ Na-phosphate (pH 7.5)) and resuspended in $1 \mathrm{ml}$ cold PBS) and incubated overnight while slowly tumbling at $4^{\circ} \mathrm{C}$. Beads were repeatedly collected $\left(2,000 \mathrm{rpm}, 1 \mathrm{~min}, 4^{\circ} \mathrm{C}\right)$ and washed with $400 \mu 1$ freshly prepared B++-buffer to purify GST-fusions and any associated proteins. Samples were denatured with $100 \mu 12 \times$ SDS loading dye at $65^{\circ} \mathrm{C}$ for $15 \mathrm{~min}$ and samples were analyzed by Western hybridization.

\subsubsection{Protein stability assay ('Promoter shut-off' experiment)}

Yeast cells were pre-grown in selective minimal medium with glucose as the carbon source to an $\mathrm{OD}_{600 \mathrm{~nm}}$ of approximately 0.6 . A $50 \mathrm{ml}$ sample (-Gal) was taken and treated as described above (section 2.2.6.1). The remaining cells were collected by centrifugation $(3,000 \mathrm{rpm}$, $5 \mathrm{~min})$ and shifted to selective medium with $2 \%(\mathrm{w} / \mathrm{v})$ galactose to induce expression of myctagged fusions under control of the GAL1 promoter. After 3 hours of induction the cells were collected and half of these trp1-deficient cells were starved for tryptophan by shifting them to minimal medium without tryptophan. After 45 minutes of starvation, 3\% (w/v) glucose was added to shut off the promoter (0-min time point) and samples were taken at the indicated time points and used for protein extract preparation (see section 2.2.6.1).

\subsubsection{Fluorescence microscopy of yeast cells}

Yeast strains were grown in appropriate selective medium to early-log until mid-log phase. 
Cells from $1 \mathrm{ml}$ of the cultures were harvested by centrifugation $(2,000 \mathrm{rpm}, 1 \mathrm{~min})$, supernatant was discarded, and the pellet was resuspended in the remaining liquid. A drop of the suspension was spread on the slide and a coverslip was placed and sealed or in case of 4',6-diamidino-2-phenylindole (DAPI) staining 1-2 $\mu 1$ of mounting medium 'Vectashield Hard Set ${ }^{\mathrm{TM}}$ with DAPI' (VeCTOR LABORATORIES InC., Burlingame, CA, USA) was added before a coverslip was placed and sealed. Cells were immediately viewed in vivo using a 'Axiovert S100' microscope (ZEISS, Göttingen, D) by either differential interference contrast microscopy (DIC) or fluorescence microscopy using a GFP filter set, a YFP filter set or a DAPI filter set (AHF ANALYSENTECHNIK AG, Tübingen, D). Cells were photographed using a 'Orca-ER' digital camera (Hammamatsu Photonics GmbH, Herrsching am Ammersee, D) and the Improvision Openlab software (IMPROviSION, Coventry, GB).

\subsubsection{Growth tests}

For spot dilution assays, yeast strains were pre-cultured to the same optical densities $\left(\mathrm{OD}_{600}=\right.$ 1) and spotted onto appropriate YNB media, as indicated. Ten-fold dilutions, starting with $3 \times 10^{4}$ cells per $20 \mu \mathrm{l}$, were spotted onto the plates, incubated for 3 days at $30^{\circ} \mathrm{C}$, and photographed under white light. For assaying growth in liquid culture, overnight cultures of the yeast strains were pre-grown in YNB medium with raffinose as carbon source, gently harvested, washed with sterile water and diluted in $100 \mathrm{ml}$ of the appropriate selective medium either with glucose or galactose as carbon source to a final $\mathrm{OD}_{600}$ of 0.1 . Cells were incubated with shaking at $30^{\circ} \mathrm{C}$. OD at $600 \mathrm{~nm}$ was measured every hour. A graph was plotted using the $\mathrm{OD}_{600}$ values and the doubling time during logarithmic growth was calculated. 


\section{Results}

\subsection{Characterization of the nuclear import of the cyclin Pcl5p in the yeast Saccharomyces cerevisiae}

Previous studies have demonstrated that the degradation of the transcription factor Gcn $4 p$ takes place in the nucleus (Pries et al., 2002). Furthermore, it was shown that the transcription factor Gcn $4 p$, the cyclin-dependent kinase (CDK) Pho85p and the Gcn4p-specific cyclin Pcl5p are nuclear localized proteins independent of the amino acid availability (Pries et al., 2002; Pries et al., 2004; Bömeke, 2006). Moreover, the nuclear localization of the cyclin Pcl5p does not require the transcription factor Gcn $4 p$, the CDK Pho85p, or the CDK inhibitor Pho81p which are all known to interact with Pcl5p (Bömeke, 2006). Manipulation of the Pcl5p localization by fusing a nuclear export sequence to Pcl5p-GFP stabilizes Gcn $4 p$ pointing out the importance of the nuclear localization of Pcl5p for efficient Gcn $4 p$ phosphorylation by Pho85p/Pcl5p. Moreover, previous studies suggested that the nuclear import of Pcl5p is mediated by the importin Kap95p (Bömeke, 2006).

Pcl5p consists of 229 amino acids. The first 60 amino acids of the $\mathrm{N}$-terminus are followed by a central domain consisting of amino acids 61-180. This domain contains the predicted cyclin box (CB) which was shown to mediate substrate specificity towards Gen4p (Bömeke, 2006; Aviram et al., 2008). The C-terminus follows the CB and is composed of the last 49 amino acids of the protein. Analysis of a set of truncated Pcl5p-GFP hybrids revealed that the N-terminus with parts of the CB (Pcl5aa1-127) localizes at the plasma membrane whereas the conserved central part of Pcl5p (aa61-180) shows dot-like aggregates distributed over the whole cell. The C-terminal part of Pcl5p, either by itself or with parts of the cyclin box, efficiently targeted the fusion protein to the nucleus suggesting that the C-terminal 49 amino acid residues of Pcl5p contain a nuclear localization sequence (NLS) (Bömeke, 2006).

\subsubsection{A C-terminal NLS is required for proper Pcl5p nuclear localization}

The C-terminal fragment of Pcl5p (aa181-229) includes a putative NLS (PVKRPRESD) between positions 207 and 215 . The structure of this motif is very much alike the classical NLS of human c-myc (PAAKRVKLD), where the basic cluster is flanked by a proline and an aspartic acid residue and which was shown to play an important role in nuclear import 
(Makkerh et al., 1996). Interestingly, this putative NLS fused to the N-terminus of GFP or to the C-terminus of the exclusively cytoplasmic protein GFP-Aro7p (Pries et al., 2002) did not cause nuclear import of the fusion proteins (Bömeke, 2006).

To investigate the importance of the putative NLS motif in the C-terminus of Pc15p, the basic cluster was mutated to alanine (PVAAPAESD) which was confirmed by sequencing. Previous studies have shown that constructs encoding Pcl5aa1-180-GFP (pME2853) or Pcl5aa61-229-GFP (pME2854) expressed under control of the MET25 promoter from a highcopy $(2 \mu \mathrm{m})$ plasmid showed nuclear staining with additional cytoplasmic and membrane dotlike staining. Three different GFP fusion constructs containing the desired mutations driven by the repressible MET25 promoter on a high copy $(2 \mu m)$ plasmid were constructed: Pc15aa1-229***_GFP (pME3577), Pc15aa61-229***_GFP (pME3370), and Pcl5aa181-229***-GFP (pME3578). To study the expression of these constructs, pME3577, pME3370, and pME3578 were transformed into pcl5 mutant cells (RH3238). Furthermore, the set of Pc15p-GFP fusions which are driven by the MET25 promoter from high-copy plasmids (Bömeke, 2006) was also transformed into the pcl5 mutant cells (RH3238) for direct comparison. Comparable expression of all Pcl5p-GFP hybrids was verified by Western analysis of equivalent amounts of total protein extracts from the obtained yeast strains using monoclonal anti-GFP antibody showing that the mutation of the putative NLS does not affect the expression of these constructs but also that the various Pc15p-GFP hybrid constructs are expressed in similar amounts (data not shown).

To study the impact of the mutated NLS motif on the sub-cellular localization of the Pcl5p-GFP hybrids, cultures of transformants expressing Pcl5aa1-180-GFP (pME3573), Pc15aa61-229-GFP (pME2854), Pc15aa1-229-GFP (pME2846), Pc15paa61-180 (pME2858), Pcl5aa1-229***-GFP (pME3577), Pc15aa61-229***_GFP (pME3370), and Pcl5aa181-229***-GFP (pME3578) were grown in YNB medium to early exponential phase and immediately used for fluorescence microscopy. Figure 8 shows that Pcl5aa1-229-GFP was nuclear localized whereas the middle part of Pcl5p (aa61-180) showed dot-like aggregates over the whole cell. Pcl5aa1-229***-GFP containing the mutated motif showed nuclear but also cytoplasmic staining with dot-like aggregates. This is similar to Pc15aa61-229-GFP without the entire N-terminus but also to Pc15aa1-180-GFP lacking the whole C-terminus even when this construct is expressed from a low-copy (CEN) plasmid (Figure. 8), which suggests that the observed localization pattern is not due to high overexpression. In contrast, Pcl5aa61-229***-GFP containing the mutated motif but lacking the $\mathrm{N}$-terminus displayed a predominant cytoplasmic staining with additional membrane dot- 
like structures (Figure 8), suggesting that the putative NLS motif plays an active role for nuclear localization. Furthermore, the localization of the C-terminal domain containing the mutated cluster fused to GFP was analyzed. This Pcl5aa181-229***-GFP is, in contrast to the non-mutated and clearly nuclear localized domain, found in the cytoplasm as well as in the nucleus, similar to GFP alone (Figure 8), corroborating the importance of the putative NLS motif.
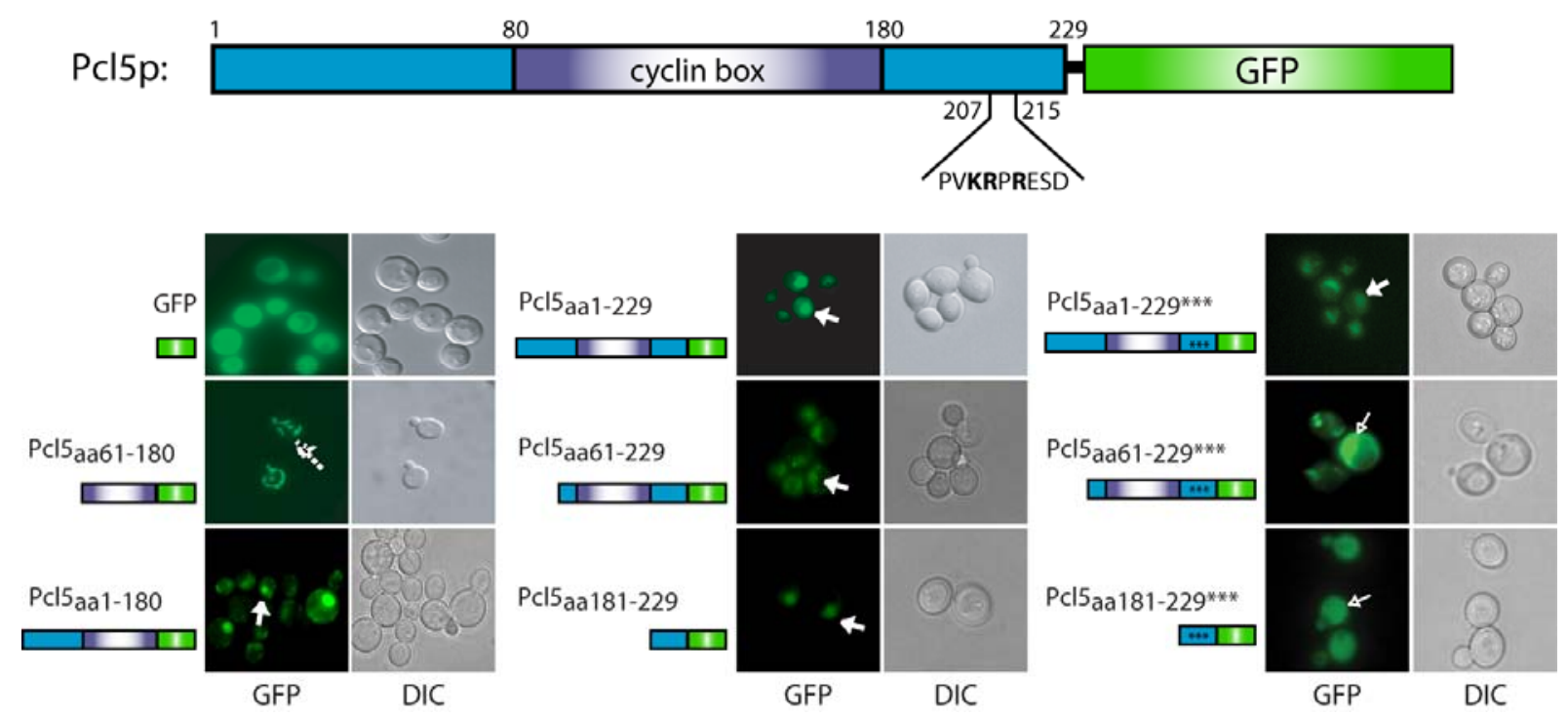

Figure 8: Mutation of a putative NLS motif in the C-terminus of Pcl5p impairs proper nuclear import. The schematic overview of Pcl5p indicates the position of the cyclin box domain mediating substrate specificity and the sequence of the putative NLS in the C-terminus (basic amino acids that are changed to alanine are shown in bold face). Yeast pcl5 mutant strain RH3238 was transformed to express either GFP alone (pME2849) or in N-terminal fusion with Pc15aa1-229 (pME2846), Pcl5aa61-229 (pME2854), Pcl5aa61-180 (pME2858), Pcl5aa181-229 (pME2859), Pc15aa1-180 (pME3573), Pcl5aa1-229*** (with mutated NLS motif marked by asterisks) (pME3577), Pc15aa61-229*** (pME3370), or Pc15aa181-229*** (pME3578) from the MET25 promoter on highcopy plasmids beside the low-copy plasmid pME3573. Living cells were visualized by differential interference microscopy (DIC) and fluorescence microscopy (GFP). Nuclear localization is indicated by white colored arrows. Accumulation in the cytoplasm is highlighted by a rendered blank arrow.

Taken together, these data indicate that aa 207 to 215 of Pc15p are necessary but not sufficient for nuclear localization of Pcl5p, but the C-terminus of Pc15p, comprising amino acid residues 181-229, acts like a non-classical NLS. 


\subsubsection{Mutation of the C-terminal NLS motif in Pcl5p partially impedes Gen4p degradation in S. cerevisiae}

The substrate specificity towards Gen $4 p$ is mediated by the cyclin box (CB) domain of Pcl5p (Bömeke, 2006; Aviram et al., 2008) and nuclear localization of Pcl5p is a prerequisite for Gcn4p degradation (Bömeke, 2006). In accordance with this, only hybrids that contained the $\mathrm{CB}$ and that showed at least in parts nuclear localization (Pcl5aa1-180-GFP and Pcl5aa61-229-GFP) were able to mediate Gcn4p degradation when expressed from the MET25 promoter on high-copy plasmids (pME2853 and pME2854, respectively) (Bömeke, 2006). For further analysis of the importance of the putative NLS motif, the activity of Pcl5aa1-229***_GFP (pME3577) and Pcl5aa61-229***_GFP (pME3370), which both include the CB domain and the mutated NLS motif, were tested with two approaches. First, Pcl5p function on Gcn $4 p$ degradation of these constructs was tested genetically by their ability to suppress Gen4p overexpression toxicity. GCN4, when highly overexpressed, inhibits cellular growth, possibly by the interference of Gen $4 p$ with other transcriptional activation pathways (Tavernarakis and Thireos, 1995). A yeast strain lacking PCL5 is hypersensitive to even moderately overexpressed GCN4 fused to the GAL1 promoter (Shemer et al., 2002). Moreover, when a functional PCL5 is expressed consecutively, the GCN4 overexpression toxicity can be suppressed, which results in restored growth of the cells under Gcn $4 p$ producing conditions in galactose medium. As shown in Figure 9 the toxicity of GCN4 moderately overexpressed from the GAL1 promoter of the low-copy plasmid pME2848 led to growth inhibition in the pcl5 background that could be suppressed by additional expression of PCL5 aa1-229-GFP (pME2846), PCL5 ${ }_{a a 61-229-G F P}$ (pME2854), or PCL5 aa1-180-GFP (pME2853) under control of the MET25 promoter from high-copy plasmids as previously described (Bömeke, 2006). Simultaneous expression of MET25-driven PCL5 aa1-229***-GFP with mutated NLS motif from the high-copy plasmid pME3577 also suppressed the GCN4 overexpression toxicity effect (Figure 9). In contrast, pcl5 mutant cells expressing PCL5 aa61229***-GFP from the high-copy plasmid (pME3370) under control of the MET25 promoter showed reduced growth like pcl5 cells expressing GFP instead of the fusion and did therefore not suppress the GCN4 overexpression toxicity effect (Figure 9). This is in agreement with the observed localization pattern of the encoded proteins shown above (Figure 8). Pcl5aa1-229***-GFP with mutated NLS motif is nuclear localized and able to suppress GCN4 overexpression toxicity like Pcl5aa1-229-GFP, Pcl5aa1-180-GFP, and Pcl5aa61-229-GFP, whereas mutation of the NLS motif in Pcl5aa61-229***-GFP without the 
entire N-terminus led to a predominant cytoplasmic localized GFP fusion protein which is unable to suppress GCN4 overexpression toxicity (Figure 9).
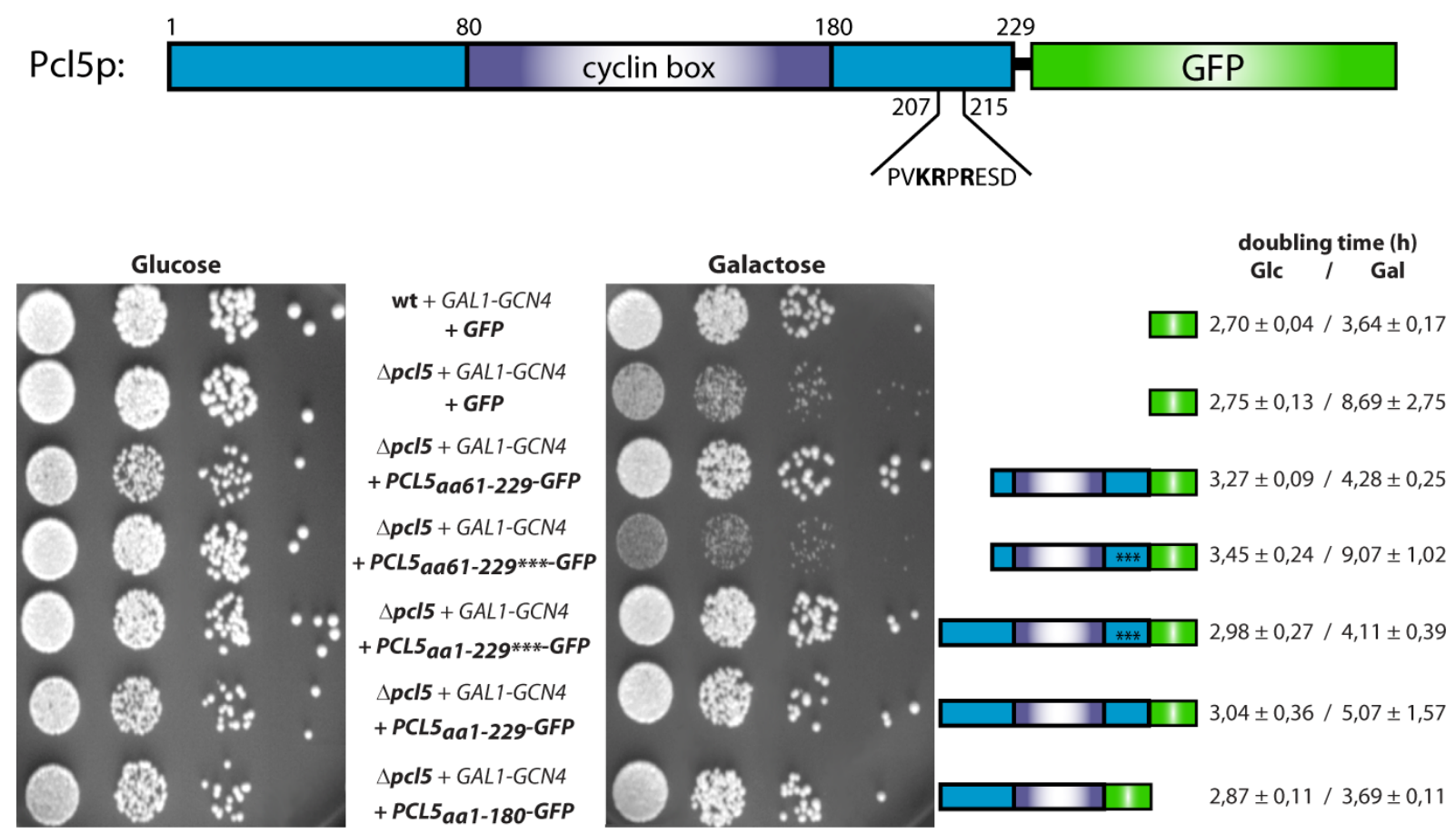

Figure 9: The putative NLS motif in the cyclin Pcl5p is required to suppress GCN4 overexpression toxicity in $S$. cerevisiae. Mutation of the putative motif leads to incapability of Pc15aa61-229*** to suppress toxicity of high amounts of Gcn4p. Yeast PCL5 wild-type cells (RH3237) expressing GAL1-driven myc3-GCN4 from the low-copy plasmid pME2848 together with MET25driven GFP on the high-copy plasmid pME2849 as well as pcl5 mutant cells (RH3238) expressing GAL1-driven myc3-GCN4 from the low-copy plasmid pME2848 together with either GFP

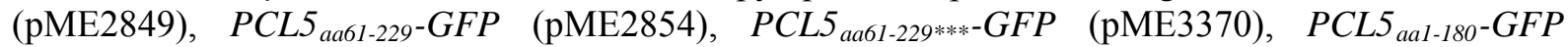

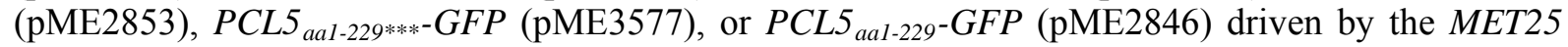
promoter on a high-copy plasmid were spotted in ten-fold dilutions on GAL1-GCN4 repressing and inducing plates with galactose or glucose, respectively. Plates were incubated for three days at $30^{\circ} \mathrm{C}$. The given doubling times (+/- standard deviation) are the average of at least three independent growth tests in liquid culture with glucose or galactose as carbon source.

For a more detailed analysis whether the mutated Pcl5p variants are able to initiate Gcn $4 p$ turnover, the myc $\mathrm{c}^{3}$-Gen $4 \mathrm{p}$ degradation was investigated. Promoter shutoff experiments with the transformants already used for the GCN4 overexpression toxicity assay were performed. As previously described and shown in Figure 10, myc ${ }^{3}-\mathrm{Gcn} 4 \mathrm{p}$ is strongly stabilized in pcl5 deficient cells compared to PCL5 wild type cells. Furthermore, constructs that were able to suppress the toxicity of high amounts of Gcn4p in growth tests were also able to initiate Gcn4p degradation in a promoter shutoff experiment. Mutation of the NLS motif in full length Pc15aa1-229***_GFP did not change rapid Gcn4p turnover compared to wild type 
Pcl5p or Pcl5aa1-229-GFP, Pc15aa1-180-GFP, or Pcl5aa61-229-GFP. Interestingly, mutation of the NLS motif in the C-terminus of Pc15aa61-229***-GFP did not completely abolish Gcn4p degradation. Although this construct was not able to suppress GCN4 overexpression toxicity, there was still very weak activity left compared to a pcl5 strain (Figure 10).
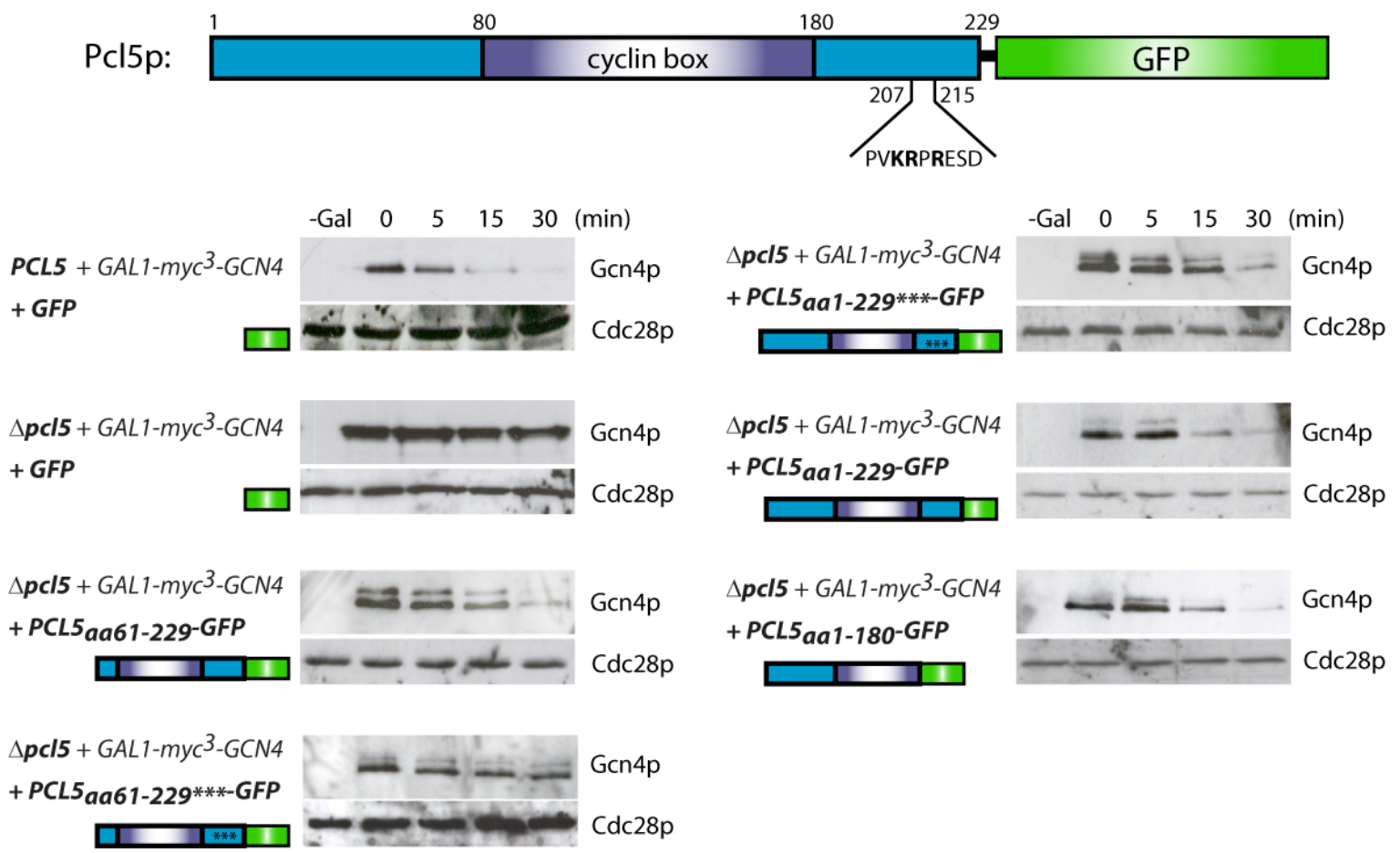

Figure 10: The C-terminal putative NLS motif in Pcl5p is required for efficient Gen4p degradation in S. cerevisiae. Pc15aa61-229***-GFP is unable to mediate proper Gcn $4 p$ degradation in GAL1 promoter shutoff experiments. Yeast PCL5 wild-type cells (RH3237) and pcl5 mutant cells were transformed to express GAL1-driven myc3-GCN4 from the low-copy plasmid pME2848 together with MET25-driven GFP on the high-copy plasmid pME2849. In addition, pcl5 mutant cells (RH3238) were transformed to express GAL1-driven myc3-GCN4 from the low-copy plasmid

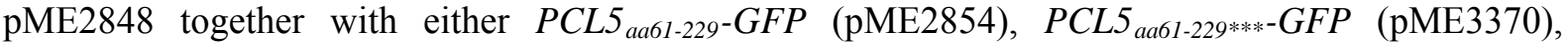

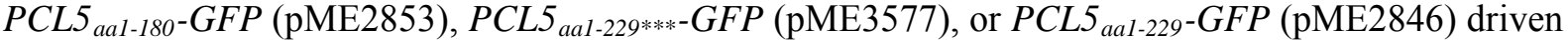
by the MET25 promoter on a high-copy plasmid. Cells were grown to early log phase in selective medium with glucose, harvested by centrifugation and incubated in minimal medium containing $2 \%$ galactose as carbon source to express $m y c^{3}$-GCN4. After $3 \mathrm{~h}$ of induction the GAL1 promoter was shut off by the addition of $2 \%$ glucose to the medium ( 0 min time point). Samples were taken at the indicated time points after addition of glucose. Levels of myc ${ }^{3}$ tagged Gcn $4 p$ were determined by immunoblotting using anti-myc antibodies. The kinase Cdc28p was used as loading control.

In summary, the results further corroborate the importance of the putative NLS motif in the C-terminus of Pcl5p for its nuclear localization which is required for initiation of the Gcn $4 p$ degradation. Loss of Pc15p nuclear localization by mutation of the putative NLS motif in the C-terminus results in loss of rapid Gen $4 p$ degradation under conditions of amino acid availability and in stabilized Gen4p. 


\subsubsection{The $\beta$ importin Kap95p imports Pcl5p into the nucleus}

In S. cerevisiae 14 different karyopherins are known to mediate the regulated transport in and out of the nucleus. So far, $13 \beta$ importins including nine import and four export receptors have been identified whereas Srplp is the only known $\alpha$ importin (Kaffman and O'Shea, 1999). Previous studies suggested that the $\beta$ importin Kap95p is involved in the efficient transport of Pcl5p into the nucleus (Bömeke, 2006). As part of a yeast $\beta$ importin mutant strain collection only cells of a kap95 temperature sensitive strain showed an accumulation in the cytoplasm at the restrictive temperature of $30^{\circ} \mathrm{C}$ of the otherwise nuclear Pcl5p-GFP. All other temperature sensitive mutants (mtr10, kap104, pse1, or pse1/kap123) and all of the strains deleted for a nonessential importin gene (kap114, kap123, nmd5, pdr6, or sxm1) showed nuclear localization of the Pcl5-GFP fusion protein. To verify that Kap95p imports Pcl5p into the nucleus it was tested whether overexpression of KAP95 is able to prevent cytoplasmic accumulation of Pcl5-GFP in the kap95 temperature sensitive mutant. Therefore, the yeast strain RH2704 (kap95ts) was transformed to express MET25 driven PCL5-GFP from the high-copy plasmid pME2846 alone or together with KAP95 under control of the MET25 promoter on the high-copy plasmid pME3583. As control, the KAP95 wild type strain RH3237 was also transformed with pME2846 and the resulting transformants were analyzed at $20^{\circ} \mathrm{C}, 30^{\circ} \mathrm{C}$, and $37^{\circ} \mathrm{C}$ using fluorescence microscopy and differential interference contrast microscopy. All transformants were pre-grown to early-log phase at $20^{\circ} \mathrm{C}$ in appropriate minimal medium before they were shifted to $30^{\circ} \mathrm{C}$ or $37^{\circ} \mathrm{C}$ for one hour to induce the temperature defect of the kap95ts strain. As shown in Figure 11A, Pcl5-GFP is nuclear localized in the wild type strain at all analyzed temperatures as well as in the kap95ts cells at $20^{\circ} \mathrm{C}$. A shift of the kap95ts strain from the permissive temperature of $20^{\circ} \mathrm{C}$ to the restrictive temperature of $30^{\circ} \mathrm{C}$ led to the described cytoplasmic accumulation of Pcl5-GFP which became in some cases more pronounced at the more restrictive temperature of $37^{\circ} \mathrm{C}$ (Figure 11A) but most of these cells looked unhealthy, with big vacuoles and aggregates or inclusions after one hour at $37^{\circ} \mathrm{C}$ (data not shown). In contrast, kap95ts cells expressing additional KAP95 showed mostly a restored nuclear localization of Pc15-GFP at the restrictive temperatures of $30^{\circ} \mathrm{C}$ and $37^{\circ} \mathrm{C}$ (Figure 11A). This shows functional complementation by the MET25 driven KAP95 and it provides further evidence that Pc15p is imported into the nucleus by the $\beta$ importin Kap95p.

The next question was whether a direct interaction between Kap95p and Pc15p can be shown by an in vivo coprecipitation assay under conditions when cellular Gen4p is rapidly degraded. 
To investigate the Kap95p/Pc15p interaction in such a 'GST pulldown', yeast pcl5 mutant cells (RH3238) were transformed to express myc ${ }^{9}$-PCL5 (pME2865) together with GSTKAP95 (pME3372) or, as controls, together with GST-PHO85 (pME2866) or GST alone (pYGEX-2T) from high-copy plasmids under control of the inducible GAL1 promoter. Expression was induced with $2 \%$ galactose in appropriate minimal medium and the fusion proteins were purified from protein extracts with glutathione sepharose beads to isolate the GST fusion and its associated proteins. Affinity purified extracts were analyzed by immunoblotting. Figure 11B shows that myc ${ }^{9}-P c 15 p$ copurified with GST-Kap95p and GSTPho85p, a known interacting protein, under the tested conditions but not with GST alone. To exclude that the physical interaction of Pc15p and Kap95p was caused by their strong overexpression, two other $\beta$ importin fusions, GST-PSE1 (pME3448) and GST-KAP104 (pME3447) were also tested concerning their interaction with the cyclin Pcl5p. Neither GST-Pse1p nor GST-Kap104p copurified myc ${ }^{9}-\mathrm{Pcl} 5 \mathrm{p}$ (Figure 11B). Additionally, the interaction of GST-Kap95p and Pcl5***-fragments with an altered C-terminal NLS motif was analyzed. Therefore, pcl5 mutant cells were transformed with GST-KAP95 or GST (pME3372 or pYGEX-2T, respectively) together with either myc ${ }^{9}-P C L 5 a a 1-229$ (pME2865), or myc ${ }^{9}$-PCL5aa1-229*** (pME3579) with mutated motif, or myc ${ }^{9}-$ PCL5aa181-229 with nonmutated (pME3580) or myc ${ }^{9}-P C L 5 a a 181-229 * * *$ with mutated motif (pME3581). As demonstrated in Fig. 11C, myc ${ }^{9}$-Pcl5aa1-229*** copurified with GST-Kap95p like the nonmutated myc ${ }^{9}-\mathrm{Pcl} 5 \mathrm{p}$, whereas neither myc ${ }^{9}-\mathrm{Pcl} 5 \mathrm{aa} 181-229$ nor myc ${ }^{9}-\mathrm{Pcl} 5 \mathrm{aa} 181-229 * * *$ interacted with GST-Kap95p under the tested conditions (data not shown). This suggests that a tight interaction between Kap95p and Pc15p requires more than the C-terminal domain of Pcl5p but it is also possible that the $\mathrm{N}$-terminal myc ${ }^{9}$-tag impairs binding of the C-terminal domain alone. 
A

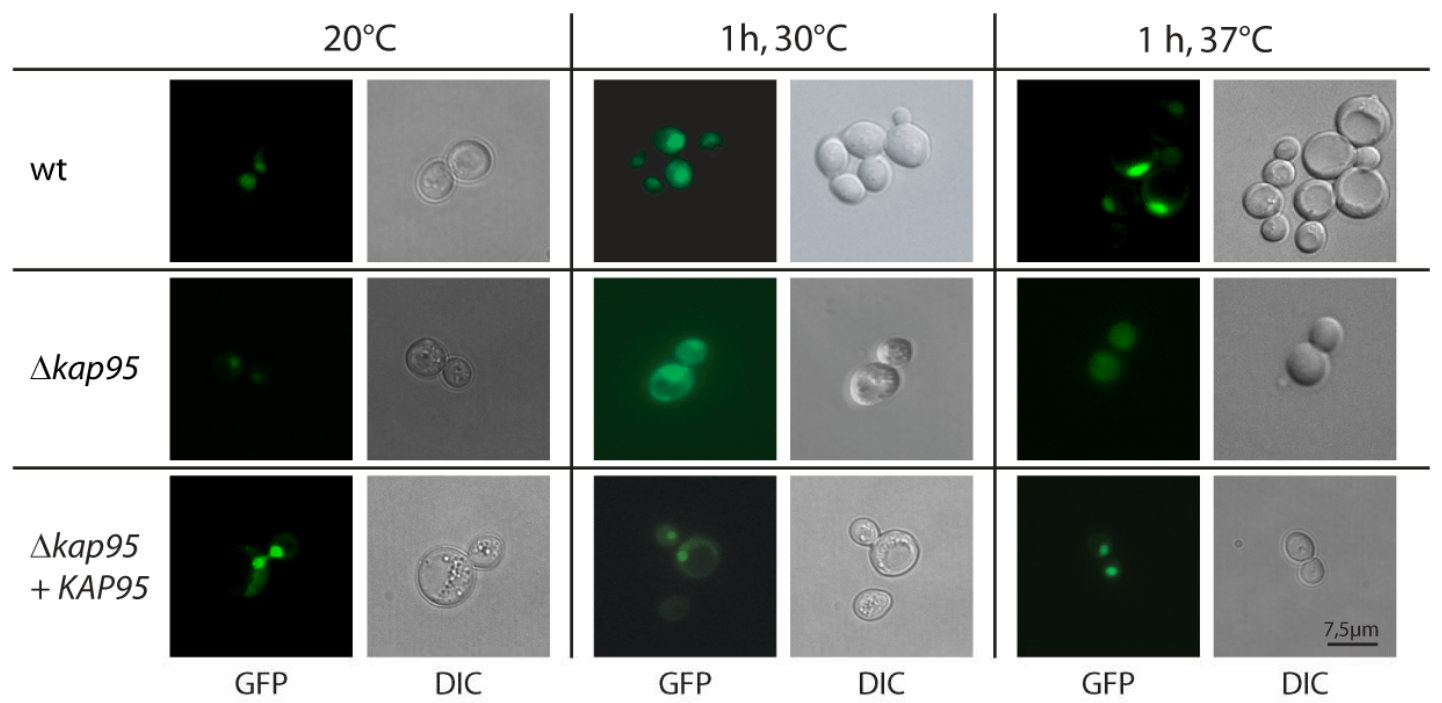

B

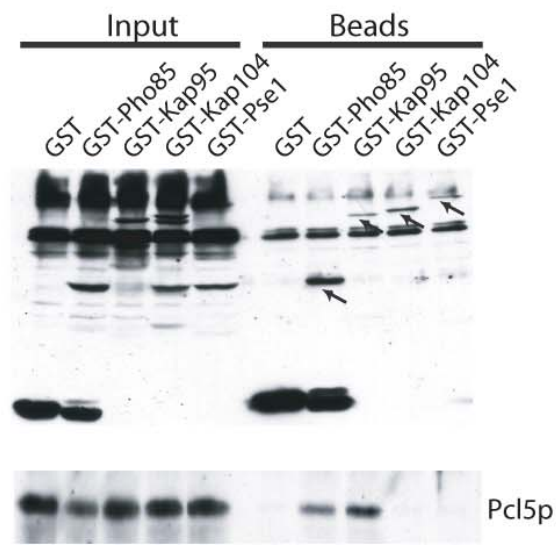

C

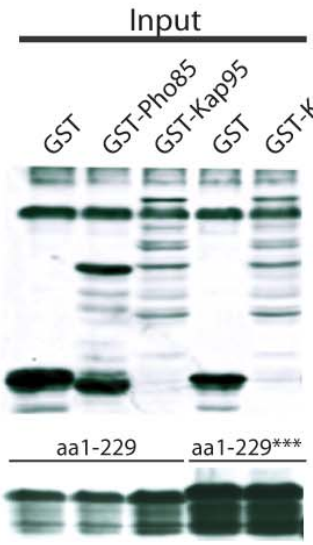

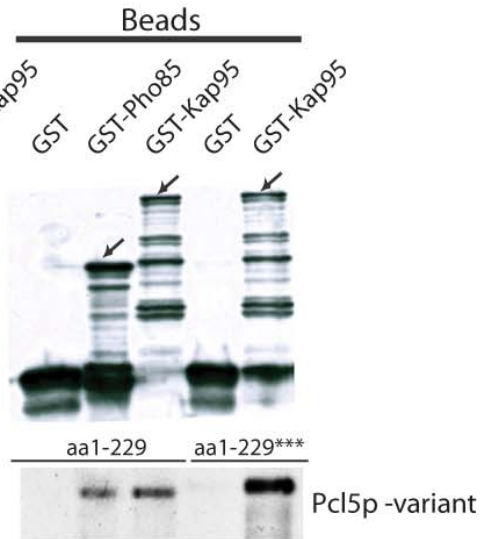

Figure 11: The nuclear transport of Pcl5p is mediated by the importin Kap95p. (A) Pcl5p nuclear translocation requires functional $\beta$ importin encoding KAP95. Nuclear import of a functional Pc15GFP fusion protein expressed from the high-copy plasmid pME2846 under control of the MET25 promoter was analyzed in KAP95 wild type cells (RH3237), and in cells with a temperature-sensitive kap95 allele (RH2704) by fluorescence microscopy (GFP) and differential interference contrast microscopy (DIC). Pcl5-GFP is nuclear localized in the wild type control independent of the temperature, whereas the kap95 mutation impaired the Pcl5-GFP nuclear import at the restrictive temperatures of $30^{\circ} \mathrm{C}$ and $37^{\circ} \mathrm{C}$. Overexpression of KAP95 driven by MET25 promoter on $2 \mu \mathrm{m}$ plasmid (pME3583) restored nuclear uptake of Pcl5-GFP at $30^{\circ} \mathrm{C}$ and $37^{\circ} \mathrm{C}$. (B) Pcl5p and Kap95p physically interact in an in vivo coprecipitation assay. Yeast pcl5 mutant strain RH3238 was transformed to express either $m y c^{9}-P C L 5$ (pME2865) with glutathione $S$-transferase (GST on pYGEX-2T), GST-KAP104 (pME3447) or GST-PSE1 (pME3448) as negative controls, or myc ${ }^{9}-P C L 5$ (pME2865) together with GST-PHO85 (pME2866) as positive control. In addition, yeast strain RH3238 was transformed to express myc ${ }^{9}$-PCL5 (pME2865) together with GST-KAP95 (pME3372). Protein levels of the fusion proteins were determined by immunoblotting using rabbit anti-GST and mouse anti-myc antibodies. The left part (Input) represents the GST, GST-Kap95p, GST-Kap104p, GST-Pse1p, and myc ${ }^{9}-\mathrm{Pcl} 15 \mathrm{p}$ before glutathione-sepharose incubation to ensure that the initial protein extracts contained similar amounts of the fusion proteins. On the right (Beads) the elutions of the beads are shown. The GST fusion proteins are marked with an arrow in the respective lane of the elutions. (C) Mutation of the C-terminal NLS motif in Pcl5p does not impede the interaction with Kap95p. Yeast pcl5 mutant strain RH3238 was transformed to express either myc ${ }^{9}$-PCL5 (pME2865) together with GST, GST-PHO85 or GST-KAP95. Additionally, pcl5 mutant cells (RH3238) were transformed to express $m y c^{9}-P C L 5^{* * *}$ with mutated NLS motif (pME3577) together with GST or GST-KAP95. 'Input' represents the initial protein extracts before and 'Beads' after glutathionesepharose incubation. Eluted GST fusion proteins are marked with arrows. 
In summary, these data demonstrate that Kap95p is required for accurate nuclear import of the yeast cyclin Pcl5p by a physical interaction. Since Pc15p does not contain a classical NLS the presented data further confirm that Kap95p interacts with a non-classical NLS.

\subsubsection{A Pho80p-Pcl5p hybrid driven by the PCL5 promoter is functional to degrade Gen4p}

It was recently shown that a hybrid consisting of the $\mathrm{N}$ - and C-terminus of the cyclin Pho80p and the cyclin box domain of Pcl5p is able to initiate Gen4p degradation (Bömeke, 2006; Aviram et al., 2008). The cyclin Pho80p normally acts as the specificity factor of the CDK Pho85p for the transcription factor Pho4 and does not mediate Gen $4 p$ specificity. Further studies revealed that a Pho80-Pc15-Pho80-GFP fusion is nuclear localized as expected and that the N-terminus of Pho80p (aa1-73) is already sufficient for nuclear import of a Pho80Pcl5-GFP fusion when overexpressed (Bömeke, 2006). To exclude the possibility that the nuclear import and the ability of Gcn4p degradation initiation of this Pho80aa1-73-Pcl5aa79-178-GFP hybrid is not due to its high overexpression, the localization was analyzed when this construct was expressed under control of the weak PCL5 promoter from a low copy plasmid (pME3576) in the pcl5 deletion strain RH3238. Since previous studies included the middle part of Pcl5p (aa61-180) but not the predicted cyclin box (aa79178) alone, the cyclin box fused to GFP (Pcl5aa79-178-GFP) expressed from the MET25 promoter on the high copy plasmid pME3574 was also analyzed. Moreover, MET25 promoter driven PCL5 aa1-180-GFP expressed from a low-copy plasmid (pME3573) was included in this study to further exclude possible overexpression artefacts.

Localization of the PCL5 promoter driven PHO80-PCL5-GFP construct was compared to chromosomally GFP tagged PCL5 of strain RH3239. Fluorescence microscopy showed the already described nuclear localization of endogenously expressed Pcl5-GFP which was confirmed for the first time by DAPI staining in this study (Figure 12). In comparison, the Pho80aa1-73-Pcl5aa79-178-GFP construct expressed from the PCL5 promoter was also predominantly nuclear localized clearly demonstrating that the nuclear import is not because of the high level of expression. As expected, the cyclin box domain (Pc15aa79-178) fused to GFP was found as aggregates over the whole cell, similar to the localization of the slightly bigger central part (aa61-180) (Figure 12 and Figure 8, respectively) further corroborating that the N-terminus of Pho80p (aa1-73) is responsible to transport the Pho80-Pc15-GFP 
hybrid into the nucleus. Expression of Pc15aa1-180-GFP from a low-copy plasmid (CEN) did also not change the observed localization pattern. Figure 12 shows that this construct still exhibits a predominant nuclear localization, confirmed by DAPI staining, with additional cytoplasmic staining and dot-like aggregates as shown before for the high-copy construct (Figure 8). Taken together, these data demonstrate that the described localization patterns are not due to overexpression or hybrid construction and that the N-terminus of Pho80p is able to confer nuclear localization of the not nuclear localized cyclin box of Pcl5p.

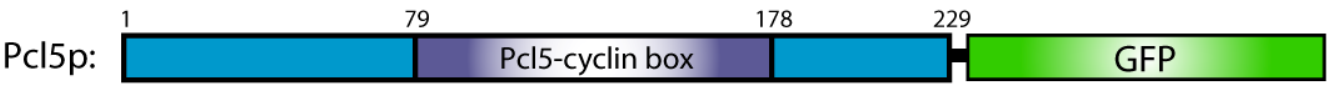

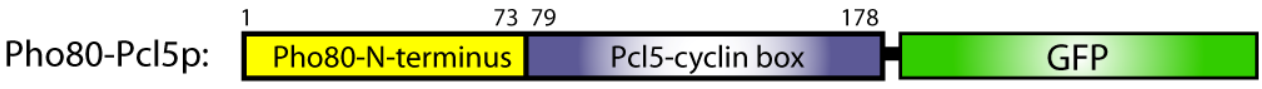

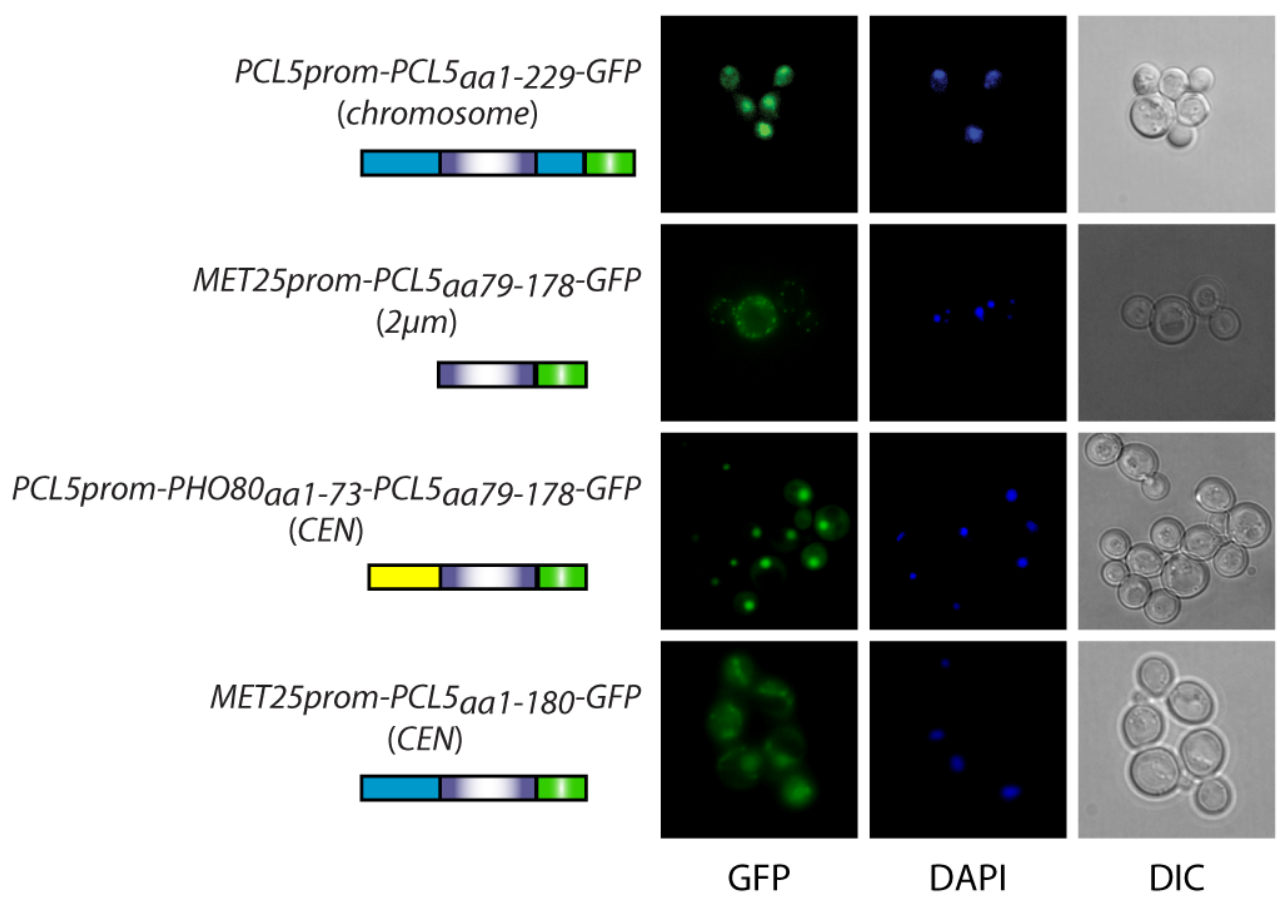

Figure 12: The N-terminus of Pho80p mediates nuclear localization of a PHO80-PCL5-GFP hybrid expressed from the PCL5 promoter. S. cerevisiae cells (RH3239) expressing PCL5-GFP from its endogenous PCL5 promoter at the chromosomal locus and pcl5 mutant cells (RH3238)

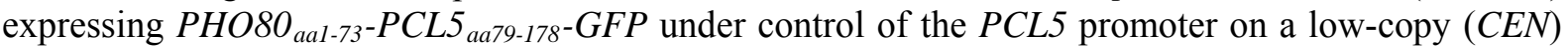
plasmid (pME3576) were analyzed by fluorescence microscopy (GFP), DAPI staining, and DIC microscopy. In addition, localization of Pcl5aa79-178-GFP expressed from the MET25 promoter on a high-copy $(2 \mu \mathrm{m})$ plasmid (pME3574) and Pc15aa1-180-GFP controlled by the MET25 promoter on the low-copy plasmid pME3573 was investigated in the pcl5 deletion strain RH3238. A schematic overview of Pcl5p and the Pho80-Pcl5p hybrid fused to GFP is given. The numbers indicate the amino acids of the corresponding protein. 
To dissect the effect of low and high levels of expression in more detail, the activity of different constructs was determined by means of GCN4 overexpression toxicity suppression as well as GCN4 promoter shutoff experiments. As controls, wild type PCL5 cells (RH3237) and pcl5 mutants cells (RH3238) were transformed to express GAL1 driven myc ${ }^{3}-G C N 4$ from the low-copy plasmid pME2848 together with MET25 driven GFP from the high-copy plasmid pME2849. Furthermore, pcl5 mutant cells (RH3238) transformed with the low-copy plasmid pME2848 to express $m y c^{3}-G C N 4$ under control of the GAL1 promoter were additionally transformed to express either PCL5-GFP (pME2846) or PCL5aa79-178-GFP (pME3574) under control of the MET25 promoter on high-copy plasmids $(2 \mu \mathrm{m})$, or

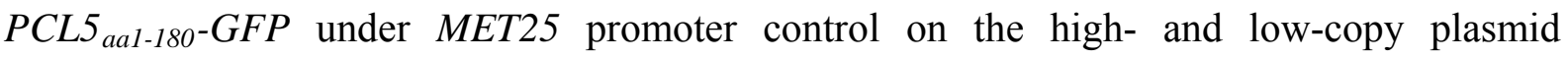
pME2853 and pME3573, respectively. In addition, pcl5 mutant cells (RH3238) were transformed to express GAL1 driven myc ${ }^{3}-G C N 4$ (pME2848) together with PHO80 $_{\text {aa1-73-PCL5 }}$ aa79-178-GFP from the high-copy plasmid pME2948 under control of the MET25 promoter or under control of the PCL5 promoter on the low-copy plasmid pME3576. As demonstrated in Figure 13, the cyclin box domain (aa79-178) fused to GFP was not able to suppress the toxicity of high amounts of Gen $4 p$ and not able to mediate myc ${ }^{3}-\operatorname{Gcn} 4 p$ degradation which is similar to Pcl5aa61-180-GFP. The middle part with the classical monopartite basic NLS of the simian virus 40 fused to the C-terminus (pME3371) was also found only as aggregates (data not shown) and did not mediate Gcn4p degradation (data not shown) suggesting that additional sequences are not only required for nuclear localization but for specific cyclin box mediated activity. The GCN4 overexpression toxicity assay furthermore revealed that the Pho80-Pcl5-GFP hybrid restored the growth inhibition of pcl5 cells even when expressed from the weak PCL5 promoter on a low copy plasmid (Figure 13). In agreement, promoter shutoff experiments showed a rapid degradation of myc ${ }^{3}-\mathrm{Gcn} 4 \mathrm{p}$ when PHO80-PCL5-GFP was expressed from the PCL5 promoter, similar to the observed Gcn4p degradation when this construct is expressed from the MET25 promoter on a high-copy plasmid. In addition, Figure 13 demonstrates that Pcl5aa1-180-GFP when expressed from a low-copy plasmid is already sufficient to overcome GCN4 overexpression toxicity and to degrade Gcn $4 p$ similar to full-length Pcl5-GFP. 
A

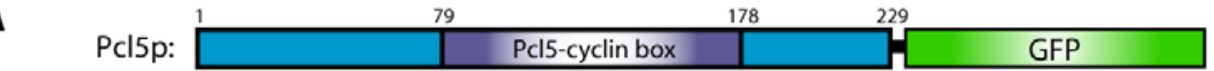

\begin{tabular}{l|l|l|} 
& & \\
& & \\
& & \\
Pho80-Pcl5p: & 79 & \\
\cline { 2 - 3 } & Pho80-N-terminus & Pcl5-cyclin box \\
\cline { 2 - 3 } & &
\end{tabular}

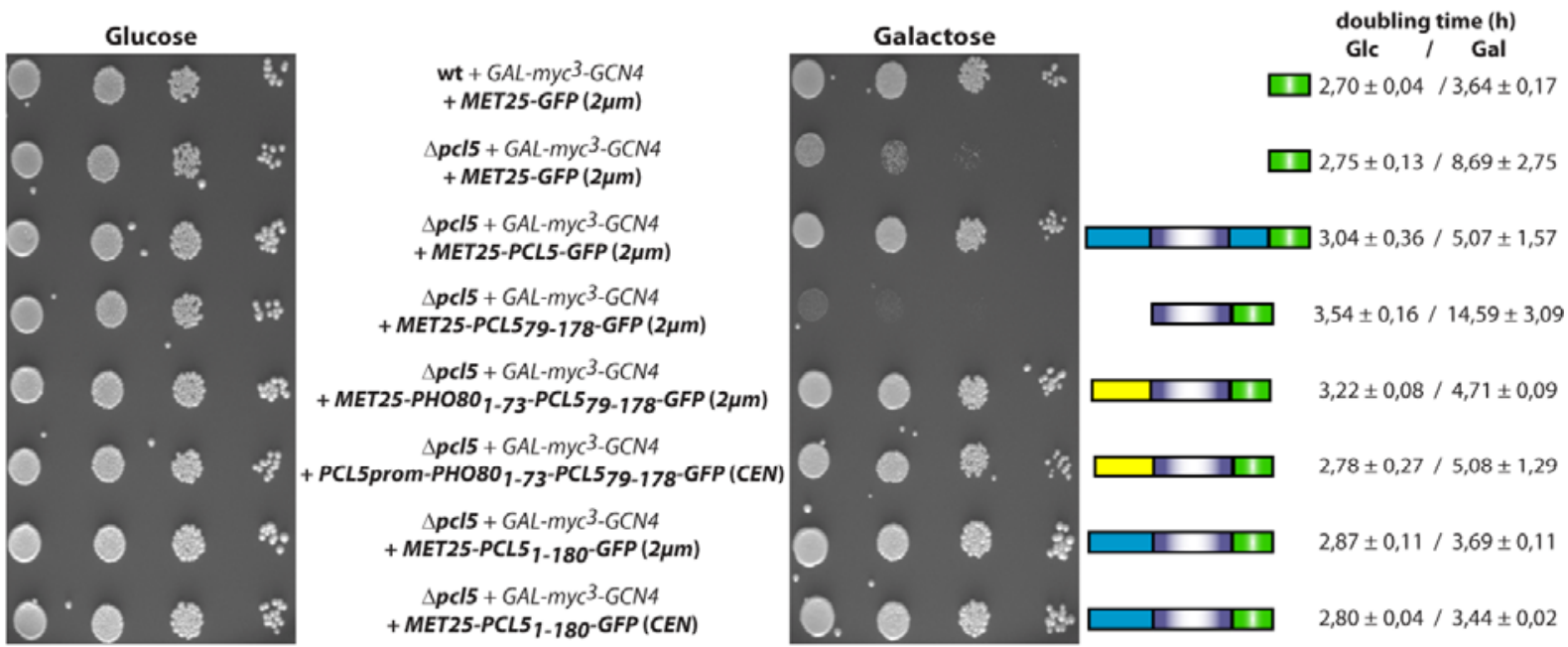

B

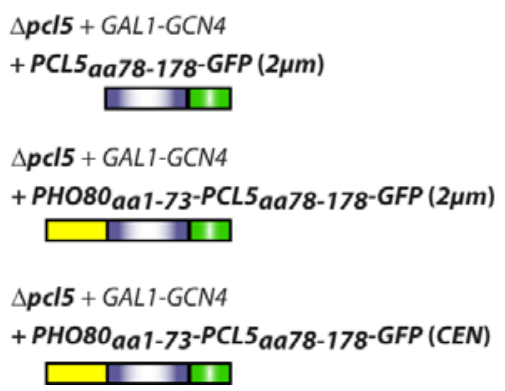

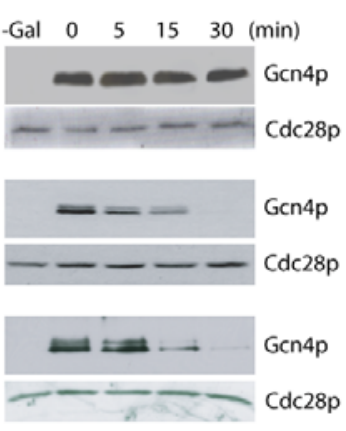

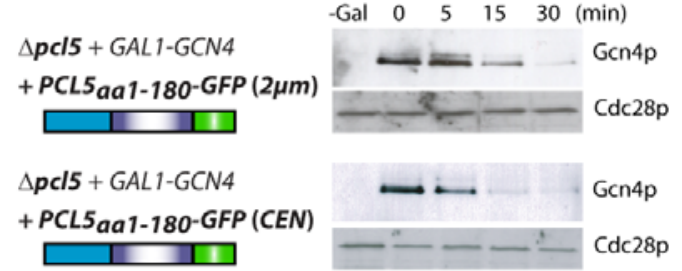

Figure 13: Functional analysis of Pcl5 hybrids fused to GFP. (A) The Pho80-Pcl5 hybrid expressed from the weak PCL5 promoter is sufficient to suppress GCN4 overexpression toxicity in yeast pcl5 cells as does the full-length Pc15p. Wild type PCL5 cells (RH3237) as well as pcl5 deficient cells (RH3238) expressing myc ${ }^{3}$-GCN4 from the GAL1 promoter (pME2848) together with MET25 promoter driven GFP (pME2849) and pcl5 mutant cells expressing myc ${ }^{3}-G C N 4$ from the GAL1 promoter (pME2848), together with MET25-PCL5 ${ }_{a a 1-229}$-GFP (pME2846, 2 $\mu \mathrm{m}$ ), MET25-PCL5 aa79-178- $^{-}$

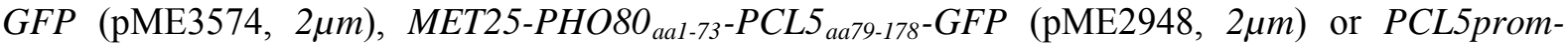
PHO80 aa1-73-PCL5 aa79-178-GFP (pME3576, CEN), and MET25-Pcl5 aa1-180-GFP (pME2853, $2 \mu \mathrm{m}$ or pME3573, CEN) were spotted in ten-fold dilutions on glucose and galactose to induce expression of GAL1 driven GCN4. Plates were photographed after 3 days incubation at $30^{\circ} \mathrm{C}$. Furthermore, these cells were used for growth tests in liquid culture with either glucose or galactose as carbon source, and the doubling times of three or more independent cultures were determined. (B) The PHO80-PCL5 hybrid is able to promote Gcn $4 p$ degradation like full-length Pcl5p. Protein amounts of myc ${ }^{3}-G c n 4 p$ and $C d c 28 \mathrm{p}$ were investigated by immunoblotting at the indicated time points after GAL1 promoter shutoff in the same transformed yeast cells described in (A).

In summary the presented data show that nuclear localization of the Pho80p-Pcl5p hybrid is mediated by the N-terminal domain of Pho80p and that this is sufficient for Gcn $4 p$ degradation similar to natively expressed Pc15p. Moreover, they confirm that the Gcn $4 p$ specificity is mediated by the cyclin box domain of Pc15p, but they also suggest that beside 
the nuclear localization function, additional Pho85-cyclin sequences might be necessary for proper cyclin box activity.

\subsection{Characterization of the Pho85p/Pcl5p interaction and the Gen $4 p$ stability in sated and amino acid starved cells of budding yeast}

In S. cerevisiae, the transcriptional activator Gen4p is subject to strict regulation on two different levels within two different compartments of the cell. Yeast Gcn4p protein synthesis is regulated in the cytoplasm whereas its stability is regulated in the nucleus. In case of sufficient amino acid supply, GCN4 mRNA is weakly translated and Gen4p is rapidly degraded. The Gen $4 p$ degradation is mediated by its phosphorylation and involves two cyclindependent kinases (CDK), Srb10p and Pho85p. Srb10p phosphorylation of Gen4p requires the cyclin Srb11p but is independent of the amino acid availability and occurs constitutively. In contrast, Pho85p mediated Gcn $4 p$ degradation depends on sufficient supply with amino acids and requires the highly instable cyclin Pc15p, which was shown to be specific for Gcn4p degradation. The initial and regulated step of the rapid Gen4p degradation is its phosphorylation at a specific residue, Thr165, by the Pho85p/Pcl5p complex (Meimoun et al., 2000; Shemer et al., 2002) leading to its ubiquitination by the $\mathrm{SCF}^{\mathrm{CDC} 4}$ mediated pathway followed by its degradation by the $26 \mathrm{~S}$ proteasome. Gcn $4 p$ stability regulation depends on this phosphorylation and occurs exclusively in the nucleus (Pries et al., 2002). Depletion of amino acids results in an increase of Gcn $4 p$ stability of the otherwise highly instable protein from a half-life of five to up to 20 minutes. The cyclin Pcl5p itself is an extremely unstable protein with a half-life of only two to three minutes under both sated and amino acid starved conditions. It was suggested, that since the overall protein synthesis is reduced in starved cells, this constitutive turnover leads to the rapid disappearance of the cyclin under amino acid starvation conditions and thereby causing Gen4p stabilization (Shemer et al., 2002; Aviram et al., 2008). It was furthermore shown, that another Pho85p interacting cyclin, Pcl7p, as well as the cyclin-dependent kinase inhibitor (CKI) Pho81p, which are both constitutively nuclear localized proteins, are required for amino acid dependent Gen $4 p$ stabilization (Bömeke et al., 2006). Moreover, interaction studies revealed that the kinase Pho85p and the CKI Pho81p are associated with Pcl5p in sated cells and additionally suggested that under conditions of amino acid depletion the Pho85p/Pc15p and the Pho81p/Pcl5p interaction is 
disrupted (Bömeke et al., 2006). In contrast, the cyclin Pcl7p was shown to interact with Pho85p and Pho81p independent of the Gen $4 p$ stability.

\subsubsection{A Pho80-Pcl5-Pho80 hybrid interacts with Pho85p and Pho81p in the presence and absence of amino acids}

As already described above (section 3.1.4.), a Pho80-Pcl5-Pho80p hybrid that contains the Pcl5-cyclin box domain, is able to mediate Gen4p degradation whereas the cyclin Pho80p is normally involved in phosphate metabolism in interaction with the CDK Pho85p and the CDK inhibitor Pho81p. Pho81p interacts with Pho85p/Pho80p independent of the phosphate conditions but only inhibits Pho85p/Pho80p activity when phosphate becomes limiting. This is in contrast to the observed disruption of the Pho85p/Pc15p and Pho81p/Pcl5p complexes in amino acid starved yeast cells (Bömeke et al., 2006). To get further insights into the mechanism of Gen4p stability regulation, i. e. whether the Pcl5-cyclin box domain is involved in the assembly/disassembly of the complexes, the interaction of the Pho80-Pcl5 hybrids with Pho85p and Pho81p was investigated by an in vivo coprecipitation assay described above under conditions of amino acid saturation and of amino acid starvation.

Tryptophan auxotrophic yeast cells of the pcl5 mutant strain RH3238 were transformed to express functional myc ${ }^{9}-$ PHO80 $_{a a 1-73}-$ PCL $_{a a 79-178}-P H O 80_{a a 170-294}$ (pME3721) together with either GST (pYGEX-2T), or GST-PHO85 (pME2866), or GST-PHO81 (pME2867) under control of the GAL1 promoter. Expression of the fusion proteins was induced in appropriate galactose minimal medium and half of the cells were shifted to medium lacking tryptophan for 45 minutes, conditions that were shown to stabilize Gcn4p. This also results in growth arrest of the cells that led to a lower amount of cells when harvested compared to the culture supplemented with tryptophan which confirmed that these cells still carried the trp1-1 mutation. Glutathione sepharose beads were used to purify the GST fusion proteins and their connected proteins from cell extracts. The extracts were analyzed by immunoblotting. As demonstrated in Figure 14A, myc ${ }^{9}$-Pho80-Pc15-Pho80p did not copurify with GST alone but was coprecipitated with GST-Pho85p and with GST-Pho81p in similar amounts under both conditions. This is significantly different from the described interaction of Pcl5p with Pho85p or Pho81p. Whereas Pcl5p only interacted with Pho85p and Pho81p in sated cells, the Pho80-Pc15-Pho80p hybrid clearly interacted in sated as well as in amino acid starved cells. To exclude unspecific coprecipitation, the beads were washed four times by vigorously 
shaking each time which results in relatively low coprecipitated fusion protein but was reproduced at least in two independent experiments. In case of GST-Pho81p expression, sometimes a band appeared which migrated similar to GST-Pho85p which seems to be a GST-Pho81p degradation product or results from partial translation of the fusion protein (Figure 14). Constructs that contained the Pc15-cyclin box (aa79-178) but only the N- or Cterminus of Pho80p were also tested for their interaction with Pho85p. Both, myc ${ }^{9}-$ Pho80aal73-Pc15aa79-178p (pME3722) and myc ${ }^{9}-P c 15 a a 79-178-P h o 80 a a 170-294 p \quad$ (pME3723) interacted with GST-Pho85p in sated as well as in amino acid starved cells similar to Pho80Pcl5-Pho80p (data not shown). As mentioned above, the cyclin Pho80p normally forms a complex with Pho81p independent of the phosphate conditions in the cell. It is therefore conceivable that the $\mathrm{N}$ - or the C-terminus of Pho80p are involved in the tight interaction between the hybrids and Pho81p under both conditions and that this in turn leads to the investigated constitutive interaction of the hybrids with Pho85p. To investigate the possibility that the found interaction of the Pho80-Pc15p hybrids with the kinase Pho85p is only due to the constitutive binding of Pho81p to these hybrid constructs, additional GST pulldown experiments were performed in the absence of PHO81. Expression of myc ${ }^{9}$-PHO80-PCL5-PHO80 (pME3721) and GST tagged PHO85 (pME2866) was induced in sated and starved pho81 mutant cells (RH3241). Induction of expression and tryptophan starvation, as well as fusion protein purification, and immunodetection were performed as described before and reproduced twice. Again, starvation resulted in growth arrest of these tryptophan auxotrophic mutants. Figure 14B demonstrates that myc ${ }^{9}-\mathrm{Pho} 80-\mathrm{Pcl} 5-\mathrm{Pho} 80$ copurified with GST-Pho85p in sated pho81 cells when Gen4p is instable. Interestingly, in amino acid starved pho81 cells, a slightly reduced amount of the myc ${ }^{9}$ epitope tagged Pho80-Pc15-Pho80p expressed from the GAL1 promoter was detected in the initial protein extract which was adjusted to the amount of the protein in sated cells. However, under amino acid starvation conditions myc ${ }^{9}$-Pho80-Pcl5-Pho80p copurified with GST-Pho85p to the same degree as under conditions of amino acid depletion (Figure 14B). This shows that the constitutive interaction of the Pho80-Pcl5-Pho80p hybrid with the CDK Pho85p is independent of the CKI Pho81p. 
A

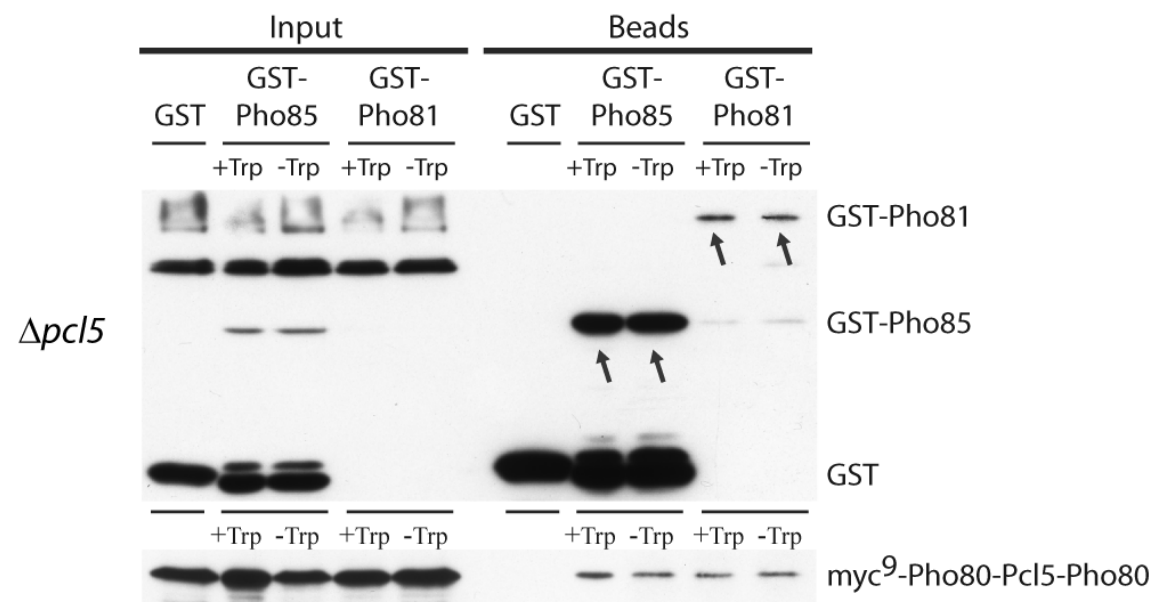

B

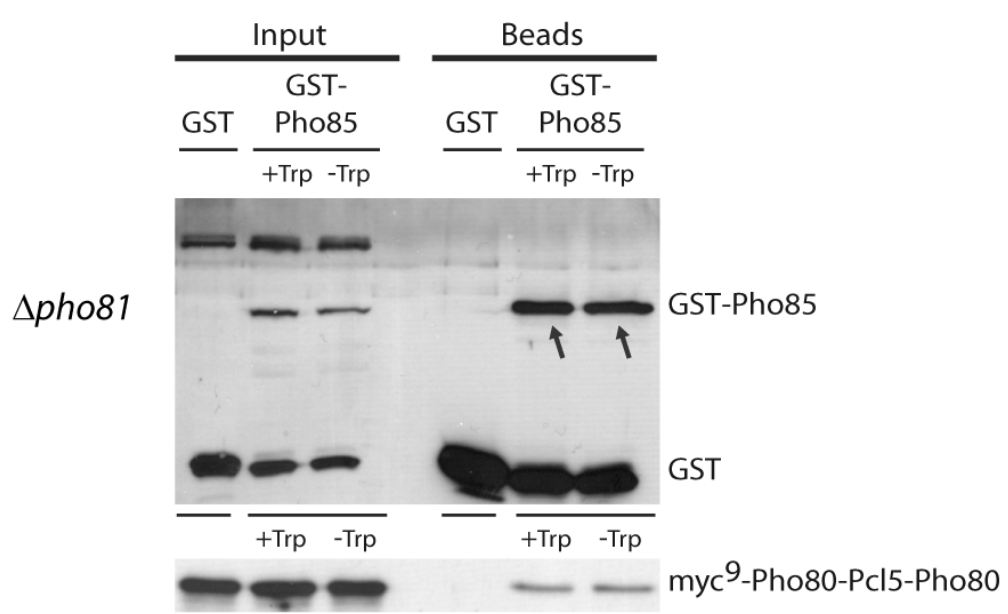

Figure 14: A Pho80-Pcl5-Pho80p hybrid constitutively interacts with Pho85p and Pho81p. (A) The tryptophan auxotrophic S. cerevisiae strain RH3238 (pcl5) was transformed to express either

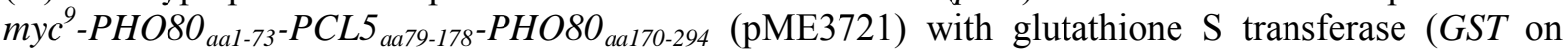
pYGEX-2T) as negative control, or with GST-PHO85 (pME2866), or together with GST-PHO81 (pME2867). Additionally (B), the tryptophan auxotrophic and pho81 deficient yeast strain RH3241 was used to express myc ${ }^{9}-P H O 80_{a a 1-73}-P C L 5_{a a 79-178}-P H O 80_{a a 170-294}$ (pME3721) together with GST (pYGEX-2T) or with GST-PHO85 (pME2866). Protein amounts of the fusion proteins were analyzed in sated (+Trp) and starved (-Trp) cells by Western hybridization with anti-myc and anti-GSTantibodies. The left part (Input) represents the GST, GST-Pho85p, GST-Pho81p and myc ${ }^{9}-P h o 80-$ Pcl5-Pho80p prior to glutathione sepharose incubation to ensure that the initial protein extracts contained equal amounts of the fusion proteins. On the right (Beads), the eluted extracts of the glutathione sepharose are shown. The GST fusion proteins are marked with an arrow in the corresponding lane of the elutions. The visible band in the GST-Pho81p lanes that migrates like GST-Pho85p (Figure A) results from GST-Pho81p degradation or incomplete translation of the expressed fusion construct.

In summary, these results verify the in vivo interaction of the Pho80-Pc15-Pho80p hybrid with the CDK Pho85p and show for the first time an interaction with the CKI Pho81p. These interactions are independent of the amino acid supply of the cells and furthermore, Pho81p is not required for the assembly of the Pho85p/hybrid complex. More important, these data suggest that the Pcl5-cyclin box domain is not sufficient to mediate the amino acid starvation 
dependent disruption of the Pho85p/Pcl5p and Pho81p/Pcl5p complexes. It is possible that the Pho80p N- or C-terminus fused to the Pc15p cyclin box lead to the constitutive binding to the CDK Pho85p and the CKI Pho81p but it seems more likely that additional parts of the cyclin Pcl5p are involved in the described disassembly of the Pho85p/Pcl5p and Pho81p/Pc15p complexes in amino acid starved cells.

\subsubsection{The $S$. cerevisiae Pho85p/Pcl5p and Pho81p/Pcl5p complexes are formed independently of the amino acid availability}

The cyclin Pcl5p was shown to interact with the CDK Pho85p as well as with the CKI Pho81p in sated yeast cells but not in amino acid starved cells (Bömeke et al., 2006). In contrast, a Pho80-Pc15-Pho80p hybrid which is able to mediate Gcn4p degradation interacted with Pho85p and Pho81p independent of the amino acid conditions. Since the middle part of Pcl5p (aa79-178) in this hybrid did not mediate the disruption of the interaction with Pho85 and Pho81p in amino acid starved cells, the $\mathrm{N}$ - or the C-terminus of Pcl5p might be required for the disassembly. Therefore, two functional Pcl5p variants were constructed that either lack the N-terminus or the C-terminus and analyzed by coprecipitation assays in at least two independent experiments as already described above. The yeast strain RH3238 (pcl5) was

transformed to express the truncations, $m y c^{9}-P C L 5_{a a 1-180}$ (pME3724) or myc ${ }^{9}-P C L 5_{a a 61-229}$ (pME3725), together with GST (pYGEX-2T), or GST-PHO85 (pME2866), or GST-PHO81 (pME2867) from the GAL1 promoter in appropriate galactose medium. Amino acid starvation was induced by shifting the tryptophan auxotrophic cells to medium lacking tryptophan for 45 minutes, conditions that were shown to induce amino acid starvation and that led to growth arrest of the cells as mentioned above. The GST fusion proteins and their associated proteins were purified by glutathione sepharose beads and analyzed by Western hybridization. Figure $15 \mathrm{~A}$ and $15 \mathrm{~B}$ show that the C-terminally truncated Pcl5aa1-180p copurified with GSTPho85p and GST-Pho81p when amino acids were available but also under conditions of amino acid deprivation. Deletion of the N-terminal domain of Pcl5p resulted in similar constitutive interaction of Pc15aa61-229p with Pho85p and with Pho81p independent of the amino acid availability (Figure 15C and 15D). This suggested that neither the C-terminal domain nor the N-terminal domain of Pcl5p alone mediates the dissociation of Pho85p/Pcl5p or Pho81p/Pc15p in starved cells. Furthermore, the observation was made that significantly less truncated Pcl5p was often detectable in the initial protein extract of amino acid starved 
cells compared to sated cells and that this difference correlated with the amount of coprecipitated protein (Figure 15).

A
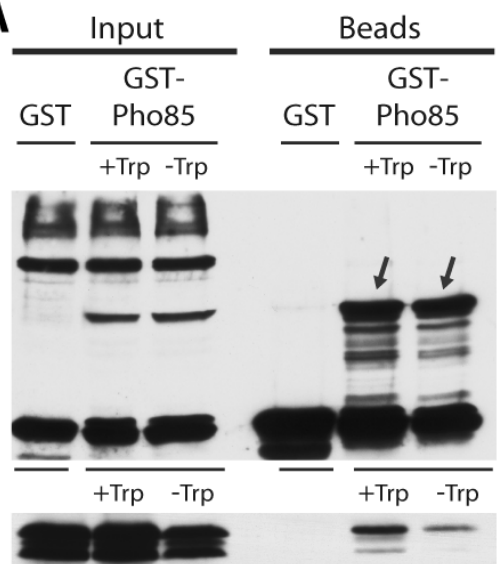

C
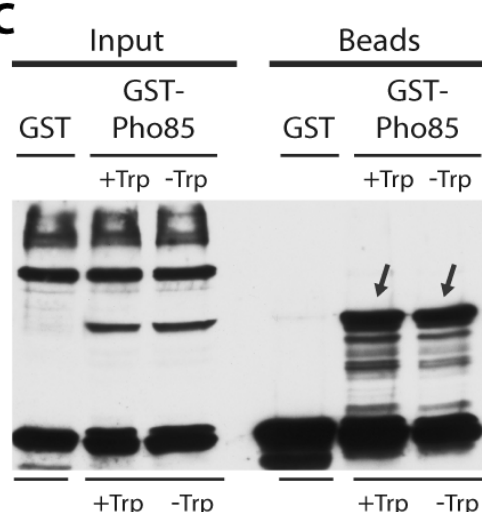

- $\frac{\operatorname{Trp}-\operatorname{Trp}}{+\cos }$
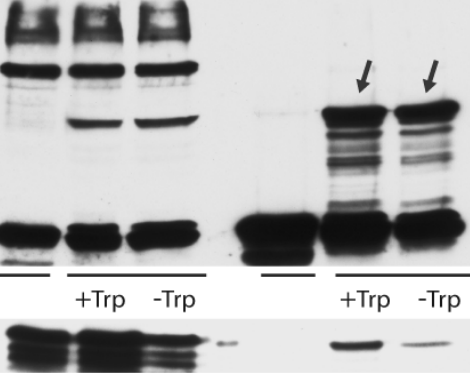

GST-Pho85

GST

- myc ${ }^{9}-$ Pcl5aa61-229
B
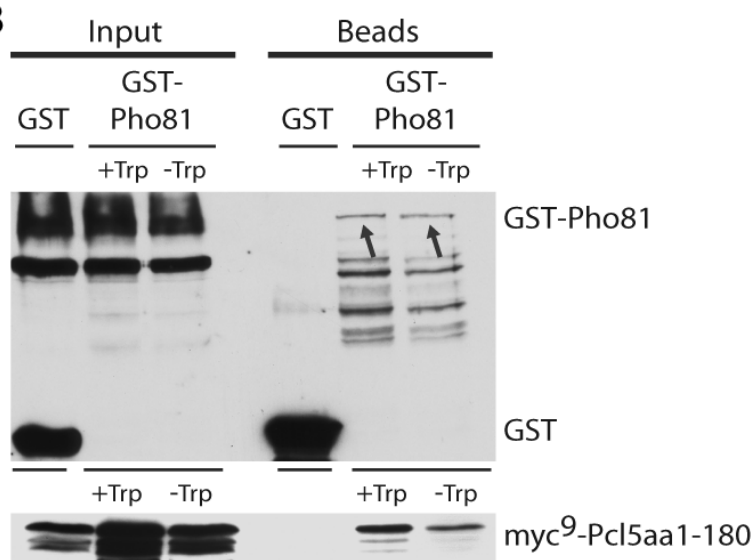

D
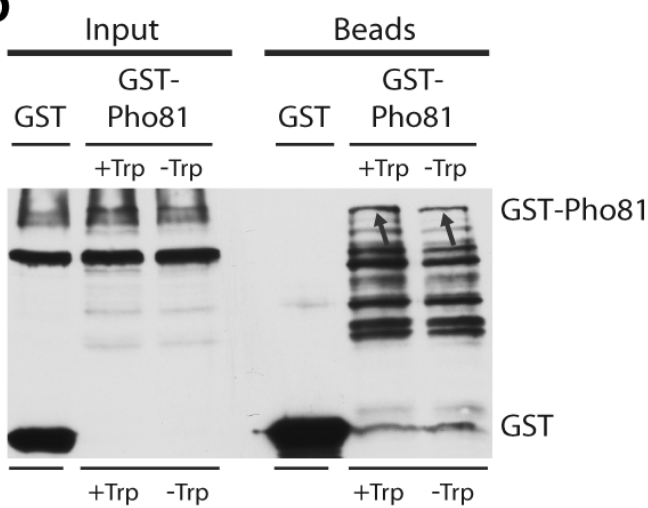

GST

myc ${ }^{9}-$ Pcl5aa61-229

Figure 15: N-terminally or C-terminally truncated Pcl5p variants interact with Pho85p and Pho81p in sated and amino acid starved yeast cells. The S. cerevisiae strain RH3238 (pcl5) was transformed to express either GST (pYGEX-2T) as negative control, GST-PHO85 (pME2866) (A), or GST-PHO81 (pME2867) (B) together with $m y c^{9}-P C L 5_{a a 1-180}$ (pME3724). The same tryptophan auxotrophic cells (RH3238) were used to express myc ${ }^{9}-P C L 5_{a a 61-229}$ (pME3725) together with GST (pYGEX-2T), with GST-PHO85 (pME2866) (C) or with GST-PHO81 (pME2867) (D). Protein amounts of the fusion proteins were analyzed in sated (+Trp) and starved (-Trp) cells by immuno blotting with anti-myc and anti-GST-antibodies. The left part (Input) always represents the GST, GSTPho85p, or GST-Pho81p and truncated myc ${ }^{9}-\mathrm{Pcl} 15 \mathrm{p}$ variant prior to glutathione sepharose incubation. On the right (Beads) the eluted extracts are shown. In the elutions (Beads), the GST fusion proteins are marked with an arrow in the corresponding lane.

These observed differences became more obvious when the time of exposure during the chemiluminescence reaction was shortened so that the strongest signal did not cause saturation of the film (data not shown). A possible explanation for this discrepancy in protein level is of course the starvation, which leads to a reduced translation of the GAL1 promoter driven constructs. Since this effect was not visible in case of stable GST-Pho85p (Figure 15A 
and $15 \mathrm{C}$ ) or $\mathrm{myc}^{9}$-Pho80-Pcl5-Pho80p (Figure 14) it was assumed that the difference in protein amount of these truncated Pcl5p variants in sated versus starved cells is due to their faster degradation. It was recently shown that the rapid and constitutive turnover of Pc15p is mediated by two independent signals, an N-terminal and a C-terminal degradation signal (Aviram et al., 2008). Loss of either or of both degradation signals should stabilize the corresponding proteins compared to full-length Pc15p. Therefore, the stability of Pho80-Pcl5Pho80p, Pc15aa1-180p, Pcl5aa61-229p and full-length Pc15p was investigated by promoter shutoff experiments. Yeast strain RH3238 (pcl5) was transformed to express either myc ${ }^{9}$ PHO80 $_{\text {aa1-73-PCL5 }}$ aa79-178-PHO80 aa170-294 $_{\text {(pME3721), myc }}{ }^{9}-$ PCL5 $_{a a 1-180}$ (pME3724), myc ${ }^{9}-$ PCL5 aa61-229 (pME3725), or myc ${ }^{9}$-PCL5 (pME2865) from the galactose inducible GAL1 promoter. The amounts of the myc ${ }^{9}$ epitope tagged proteins were analyzed after GAL1 promoter shutoff in sated and starved cells. Starvation was induced by a shift of these tryptophan auxotrophic cells to medium without tryptophan for $45 \mathrm{~min}$. As expected and demonstrated in Figure 16, myc $^{9}$-Pho80-Pcl5-Pho80p was very stable and almost not degraded after 60 minutes whereas the truncated Pcl5p variants, myc ${ }^{9}-$ Pcl5aa1-180p and myc ${ }^{9}-$ Pcl5aa61-229p, were much more instable and hardly detectable after 45 minutes. In contrast, the full-length myc ${ }^{9}-\mathrm{Pcl} 5 \mathrm{p}$ was rapidly degraded within 15 minutes after promoter shutoff. Furthermore, amino acid starvation did not influence the stability of the tested Pcl5p versions (Figure 16).
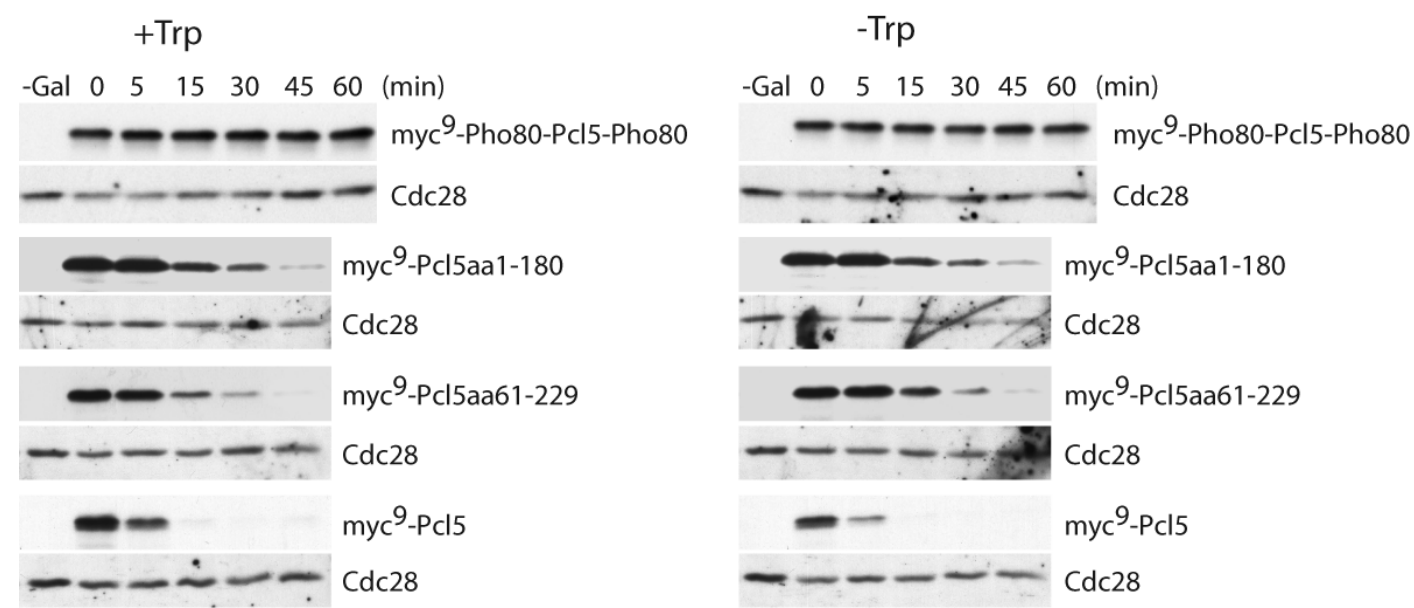

Figure 16: Analysis of the stability of myc'-Pcl5p hybrids. The tryptophan auxotrophic pcl5 mutant strain (RH3238) was transformed to express either myc ${ }^{9}-P H O 80_{a a 1-73}-P C L 5_{a a 79-178}-P H O 80_{a a 170-294}$ (pME3721), myc ${ }^{9}-P C L 5_{\text {aa1-180 }}$ (pME3724), myc ${ }^{9}-P C L 5_{\text {aa61-229 }}$ (pME3725), or myc ${ }^{9}-P C L 5$ (pME2865) controlled by the GAL1 promoter. Cells were grown to early log-phase in selective medium containing glucose as carbon source. Cells were collected by centrifugation and incubated in medium with $2 \%$ galactose to express $\mathrm{myc}^{9}$ fusion proteins. After 3 hours of induction each half the cells were transferred to medium with or lacking tryptophan to induce starvation for 45 minutes before the GAL1 promoter was turned off by the addition of $2 \%$ glucose to the medium ( 0 min time point). Protein levels of myc ${ }^{9}$ tagged Pcl5p hybrids and Cdc28p as loading control were determined in sated (+Trp) and amino acid starved (-Trp) cells at the indicated time points after GAL1 promoter shutoff. 
These results corroborate the hypothesis that the difference in protein amounts of myc ${ }^{9}-$ Pcl5aa1-180p and myc ${ }^{9}-$ Pcl5aa61-229p in sated compared to starved cells might be due to the reduced translation in starved cells combined with the rapid degradation of these proteins. Since myc ${ }^{9}-\mathrm{Pcl} 5 \mathrm{p}$ is even more unstable than the truncated versions that each lack one degradation signal, and the fact that a similar initial protein amount can only be ensured as long as the detection takes place in the unsaturated range of the used detection method led to the question raise whether the observed disruption of Pho85p/Pcl5p and Pho81p/Pcl5p complexes under amino acid starvation conditions might be caused by so far undetected difference of Pcl5 protein amounts in the initial cell extracts of sated and starved cells.

Therefore, the interaction of the highly instable cyclin Pcl5p with the CDK Pho85p and the CKI Pho81p was performed as previously described but with the difference that the initial Pcl5p amount in sated and starved cells was determined by 'unsaturated' Western hybridization and quantified, to adjust the amount of protein extracts eluted from the beads according to the amount of myc ${ }^{9}-\mathrm{Pcl} 5 \mathrm{p}$ in the initial extracts. The tryptophan auxotrophic S. cerevisiae strain RH3238 (pcl5) was transformed with myc ${ }^{9}$-PCL5 (pME2865) together with either GST (pYGEX-2T), GST-PHO85 (pME2866), or GST-PHO81 (pME2867) and the expression of these GAL1 driven constructs was induced by galactose as carbon source in the presence of tryptophan in the medium. Induction of starvation, protein purification and detection were accomplished as described before. Starvation for tryptophan was again confirmed by the fact that this results in growth inhibition of the cells as already described above. As expected, myc ${ }^{9}-\mathrm{Pcl} 5 \mathrm{p}$ copurified with GST-Pho85p and with GST-Pho81p in sated cells but not with GST alone (Figure 17). Interestingly, adjustment of the eluted protein extracts according to the abundance of myc ${ }^{9}-\mathrm{Pcl} 5 \mathrm{p}$ in the initial extracts had a significant effect. As shown in Figure 17 and confirmed by two independent experiments, myc ${ }^{9}-\mathrm{Pcl} 5 \mathrm{p}$ copurified with GST-Pho85p and GST-Pho81p even after 45 minutes of tryptophan starvation and did therefore not dissociate under starvation conditions as suggested by previous studies. 


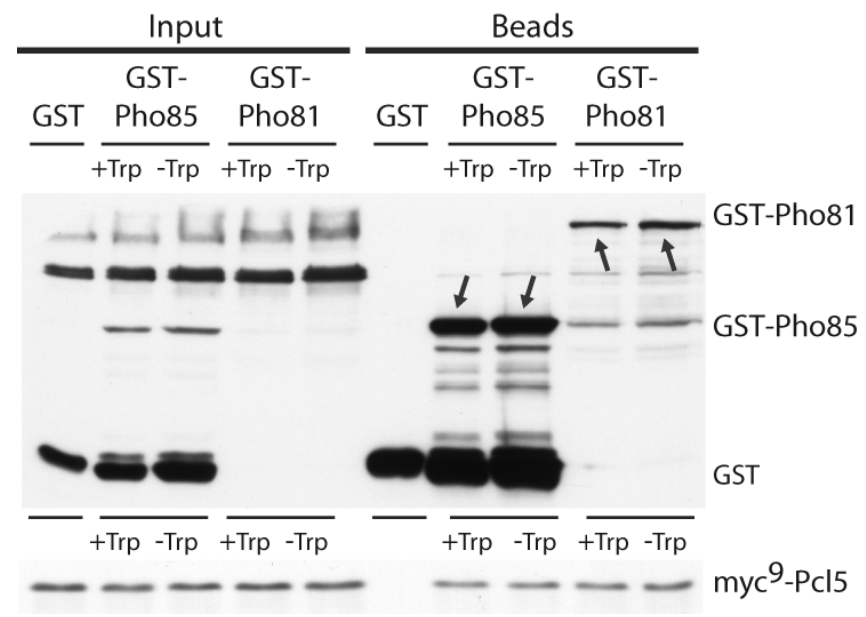

Figure 17: Pcl5p interacts with Pho85p and Pho81p in the presence and absence of amino acids. The yeast strain RH3238 ( $p c l 5$ ) was transformed to express myc ${ }^{9}-P C L 5$ together with either GST (pYGEX-2T) as negative control, or GST-PHO85 (pME2866), or GST-PHO81 (pME2867). Protein amounts of the fusion proteins were analyzed in sated (+Trp) and starved (-Trp) cells by immuno hybridization with anti-myc and anti-GST-antibodies. The left part (Input) shows myc ${ }^{9}$-Pc15p, GST, GST-Pho85p or GST-Pho81p before incubation with glutathione sepharose beads. On the right (Beads) the extracts eluted from the sepharose are shown. The GST fusion proteins are marked with an arrow in the corresponding lane of the bead extracts.

The presented data show that both complexes, Pho85p/Pc15p and Pho81p/Pcl5p, are formed to a similar degree independently of the amino acid supply as long as equal levels of the cyclin Pcl5p are available.

\subsubsection{Constitutive formation of Pho85p/Pcl5p and Pho81p/Pcl5p complexes is independent of each other and does not require Pcl7p}

It was previously shown that the assembly of Pho85p/Pcl5p in sated yeast cells does not require the CKI Pho81p (Bömeke et al., 2006). It was furthermore shown that another Pho85p and Pho81p interacting cyclin, Pcl7p, is required for Gcn $4 p$ stabilization but not for Pho85p/Pcl5p and Pho81p/Pcl5p interaction under conditions of rapid Gen4p degradation (Bömeke et al., 2006). The results presented above have shown that the kinase Pho85p as well as the CKI Pho81p interact with the cyclin Pcl5p even under amino acid starvation conditions. It was therefore assumed that Pcl7p and Pho81p are not required for the above described associations of Pc15p in amino acid starved cells. To confirm this presumption and for further characterization, the Pc15p complex formation with Pho85p and Pho81p was analyzed in cells lacking PCL7 as well as in cells deleted for PHO81 or PHO85, respectively 
and which all are tryptophan auxotrophs. Therefore, the yeast strain RH3255 (pcl7) was transformed to express $m y c^{9}-P C L 5$ (pME2865) together with GST (pYGEX-2T), with GSTPHO85 (pME2866) or with GST-PHO81 (pME2867). Additionally, the pho81 mutant strain RH3241 was transformed either with GST (pYGEX-2T) or GST-PHO85 (pME2866) together with myc ${ }^{9}$-PCL5 (pME2865). Furthermore, expression of myc ${ }^{9}$-PCL5 (pME2865) was induced together with GST (pYGEX-2T), or with GST-PHO85 ${ }^{E 53 A}$ (pME3726) encoding a GST tagged inactive variant of Pho85p, or with GST-PHO81 (pME2867) in pcl5 pho85 double mutant cells (RH3393). Expression, tryptophan starvation of these tryptophan auxotrophic cells, purification, and detection were conducted as described before. Figure 18A demonstrates that myc ${ }^{9}-P c 15 p$ interacted with GST-Pho85p and with GST-Pho81p in pcl7 deficient cells independent of the amino acid availability confirming that Pcl7p is neither required for Pho85p/Pc15p and Pho81p/Pcl5p assembly in the presence of amino acids as suggested by Bömeke et al. (2006) nor in the absence of amino acids. Similarly, deletion of PHO81 did not impair the interaction of Pho85p with Pc15p under amino acid rich conditions as described before (Bömeke et al., 2006). Although much less myc ${ }^{9}$-Pcl5p was detectable in the initial protein extracts of starved pho81 cells compared to sated cells, which made it more difficult to adjust the amount of myc ${ }^{9}-\mathrm{Pcl} 5 \mathrm{p}$ to a similar and detectable level (data not shown), myc ${ }^{9}-\mathrm{Pc} 15 \mathrm{p}$ copurified with GST-Pho85p in the absence of Pho81p under starvation conditions like under non-starvation conditions (Figure 18B). Vice versa, Pcl5p/Pho81p complex formation does not require Pho85p. Under amino acid rich and depletion conditions, myc ${ }^{9}-\mathrm{Pcl} 5$ p copurified with GST-Pho81p in cells lacking PHO85 as depicted in Figure 18C. Furthermore, the inactive kinase mutant GST-Pho $85^{\mathrm{E} 53 \mathrm{~A}} \mathrm{p}$ expressed in pho85 pcl5 double mutant cells did copurify myc ${ }^{9}$ tagged Pcl5p in the presence of tryptophan as well as in the absence of tryptophan in the medium (Figure 18C). Therefore the activity of the kinase Pho85p is not required for its interaction with the cyclin Pcl5p. 
A

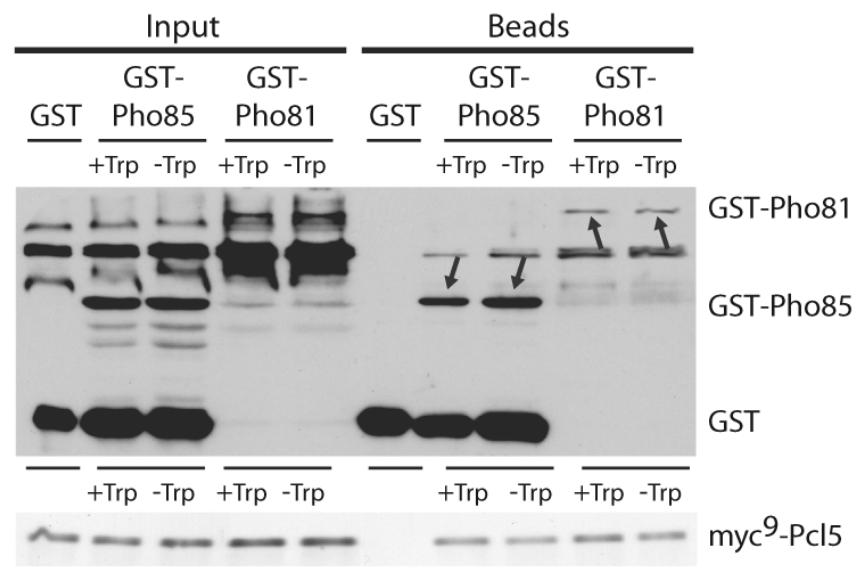

B

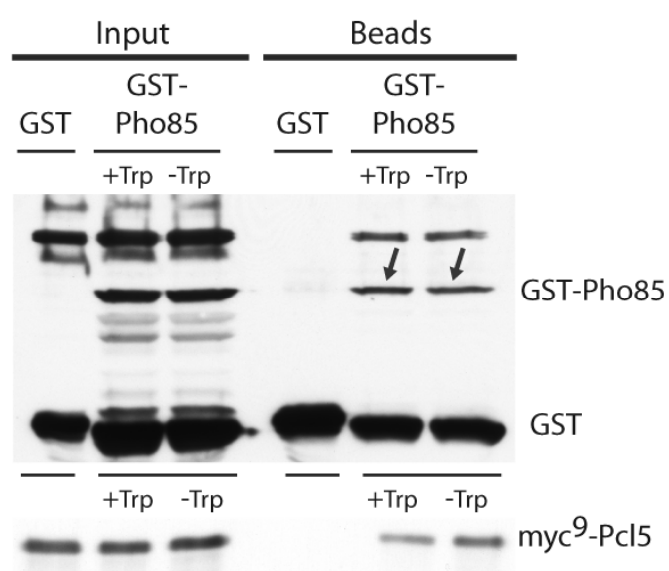

C

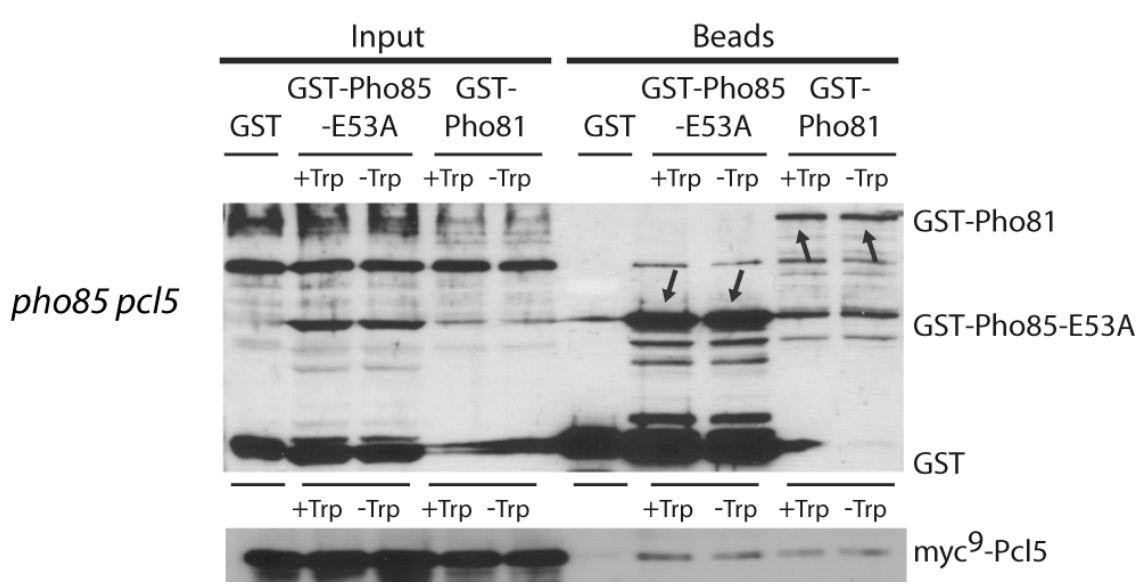

Figure 18: Protein-protein interaction of Pcl5p with Pho85p and Pho81p is independent of PCL7 and does not require Pho81p or Pho85p, respectively. (A) The tryptophan auxotrophic $S$. cerevisiae strain RH3255 (pcl7) was transformed to express myc - PCL5 together with either GST (pYGEX-2T) as control, or GST-PHO85 (pME2866), or GST-PHO81 (pME2867) and (B) tryptophan auxotrophic yeast pho81 mutant cells (RH3241) were transformed to express myc ${ }^{9}$-PCL5 with GST (pYGEX-2T) or GST-PHO85 (pME2866). Protein amounts of the fusion proteins were analyzed in sated (+Trp) and starved (-Trp) cells by Western hybridization with anti-myc and anti-GST-antibodies. The left part (Input) represents myc ${ }^{9}-P c 15 p$, GST, GST-Pho85p or GST-Pho81p before incubation with glutathione sepharose beads. On the right (Beads) the extracts eluted from the sepharose are shown. The GST fusion proteins are marked with an arrow in the corresponding lane of the bead extracts. (C) Similar experiment as described above but with the tryptophan auxotrophic pcl5 pho85 double mutant strain (RH3393) expressing myc ${ }^{9}$-PCL5 together with either GST (pYGEX-2T), or inactive kinase encoding GST-PHO85 ${ }^{E 53 A}$ (pME3726), or GST-PHO81 (pME2867). 
These findings confirm that the assembly of Pho85p/Pcl5p and Pho81p/Pcl5p takes place independently of the amino acid supply of the cells and corroborate that Pcl7p, which was shown to be involved in Gen $4 p$ stabilization, is not directly involved in formation of these complexes. Moreover, the data demonstrate that Pho85p/Pcl5p association neither requires the kinase activity nor the CKI Pho81p. Conversely, Pho81p/Pcl5p complex formation is independent of Pho85p. Therefore, it seems more likely that in a possible ternary complex of cyclin, CDK, and CKI, the kinase Pho85p and the inhibitor Pho81p are bound to the cyclin Pcl5p rather than Pho85p/Pho81p interact and Pcl5p is bound to one of them.

\subsubsection{Overexpression of PCL5 impairs the stabilization of Gen4p in amino acid starved yeast cells}

Amino acid dependent degradation of yeast Gen $4 p$ was shown to be mainly dependent on the CDK Pho85p and its activating cyclin Pcl5p (Shemer et al., 2002). It was furthermore shown that the amount of the constitutively highly unstable cyclin Pcl5p is decreased in amino acid starved cells (Shemer et al., 2002) although PCL5 is transcriptionally induced by Gen4p as response to starvation conditions (Jia et al., 2000). Pc15p was therefore suggested as an amino acid sensor via its translation efficiency. Moreover, it was shown that the cyclin Pcl5p interacts with the CKI Pho81p and that this inhibitor is required for stabilization of Gcn $4 p$ in starved cells (Bömeke et al., 2006). It was also suggested that the Pcl5p complex formation with the known interacting proteins Pho85p and Pho81p is disrupted in amino acid starved cells and that this initiates the Gcn4p stabilization (Bömeke et al., 2006). As shown above, Pho85p/Pcl5p and Pho81p/Pcl5 complexes are formed in the presence and absence of amino acids suggesting that Gen $4 p$ is not stabilized when PCL5 is constitutively overexpressed. To test this, the Gen $4 p$ degradation was investigated by promoter shutoff experiments. Yeast PCL5 strain RH3237 was transformed to express myc ${ }^{3}$-GCN4 (KB294) from the GAL1

promoter together with either MET25 promoter driven myc ${ }^{9}$-PCL5 from the low-copy plasmid (pME3727) or together with an empty vector as control (pRS415MET25). Additionally, pcl5 mutant cells (RH3238) were transformed to express myc ${ }^{3}-G C N 4$ (KB294) from the GAL1 promoter together with PCL5-GFP (pME2846) under control of the MET25 promoter on a high-copy plasmid. Promoter shutoff experiments were performed as previously described. Cells were pre-grown to early log-phase in appropriate minimal medium with glucose and shifted to medium containing galactose as only carbon source to induce expression 
$m y c^{3}-G C N 4$. After 3 hours of induction half of the tryptophan auxotrophic cells were shifted to medium with tryptophan or lacking tryptophan to induce amino acid starvation for 45 minutes before the GAL1 promoter was shutoff by the addition of $2 \%$ glucose. Protein levels of myc $\mathrm{c}^{3}-\mathrm{Gcn} 4 \mathrm{p}$ were determined in sated and starved cells after promoter shutoff. Figure 19A demonstrates that constitutive overexpression of PCL5 in wild-type and pcl5 mutant cells impaired Gcn $4 p$ stabilization in amino acid starved cells in comparison to stabilization of Gcn4p in wild-type PCL5 cells without additional copies of PCL5. Furthermore, Gen4p is rapidly and similarly degraded in sated and starved cells when PCL5 is constitutively expressed with a half-life of only a few minutes (Figure 19A). To exclude the possibility that overexpression of PCL5 impairs the general amino acid control network, which regulates the translation of natively expressed Gen $4 p$, the level of phosphorylated translation initiation factor eIF2 $\alpha$ was analyzed. Starvation for amino acids was shown to trigger the phosphorylation of eIF $2 \alpha$ by the sensor kinase Gen2p. Therefore, the level of eIF $2 \alpha-P$ should be increased after 45 minutes of starvation. As demonstrated in Figure 19A, the level of eIF2 $\alpha-\mathrm{P}$ was increased in starved cells compared to sated cells even when PCL5 was overexpressed indicating that the general amino acid control network was not impaired. These data show that Gcn $4 p$ degradation is initiated in starved cells when Pcl5p is available and provide further evidence that $\mathrm{Pho} 85 \mathrm{p} / \mathrm{Pcl} 5 \mathrm{p}$ interact in amino acid starved cells.

In a second approach, the influence of overexpressed PCL5 on resistance to 3-aminotriazole (3AT), a competitive inhibitor of the HIS3 encoded imidazole glycerol phosphate dehydratase was analyzed. Resistance to 3AT is mediated by Gen4p through activation of the HIS3 gene. It was previously reported that high overexpression of PCL5 from the GAL1 promoter led to an increase in $3 \mathrm{AT}$ sensitivity but Gcn $4 p$ was reported to be still stabilized in starved cells (Shemer et al., 2002). This in contrast to the data presented above. Therefore the growth of PCL5 wild-type cells (RH3237) and pcl5 deletion cells (RH3238) transformed to express MET25 driven myc ${ }^{9}$-PCL5 from the low-copy plasmid pME3727 or PCL5-GFP from the high-copy plasmid pME2846 was analyzed with increasing amounts of 3AT. As shown in Figure 19B, overexpression of PCL5 led to an increased and similar sensitivity of wild-type and pcl5 mutant cells to 3AT as expected and consistent with the above described results on Gcn4p degradation. Simultaneous overexpression of functional PHO81-GFP (pME2228) and myc ${ }^{9}$-PCL5 (pME3727) had no effect on increased sensitivity to 3AT (data not shown). Furthermore, the sensitivity towards 3AT was much more pronounced when PCL5-GFP was expressed from the high-copy plasmid $(2 \mu \mathrm{m})$ compared to myc ${ }^{9}$-PCL5 expressed from a lowcopy plasmid (CEN) (Figure 19B). 
A

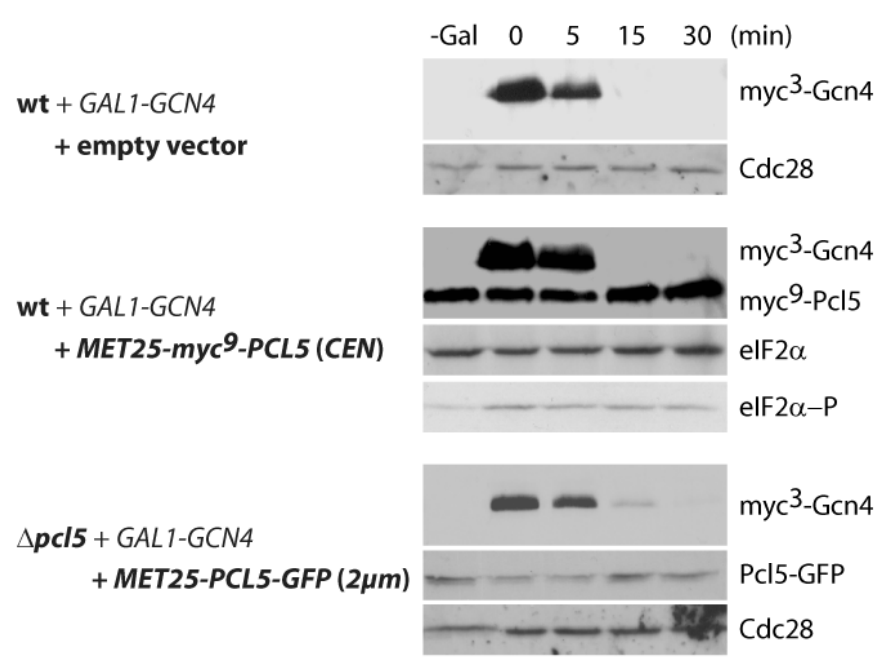

$-\operatorname{Trp}$
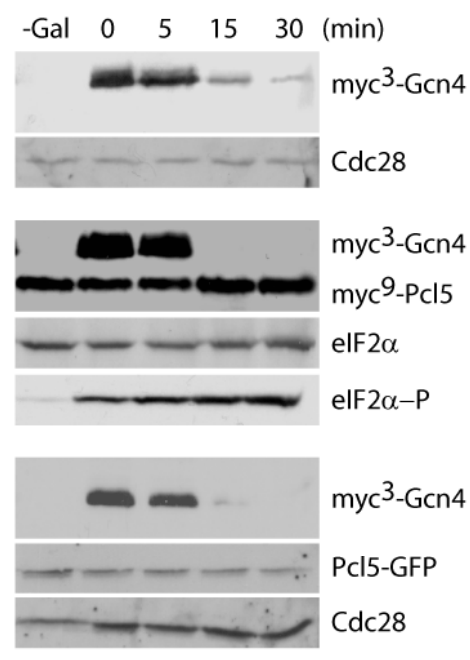

B

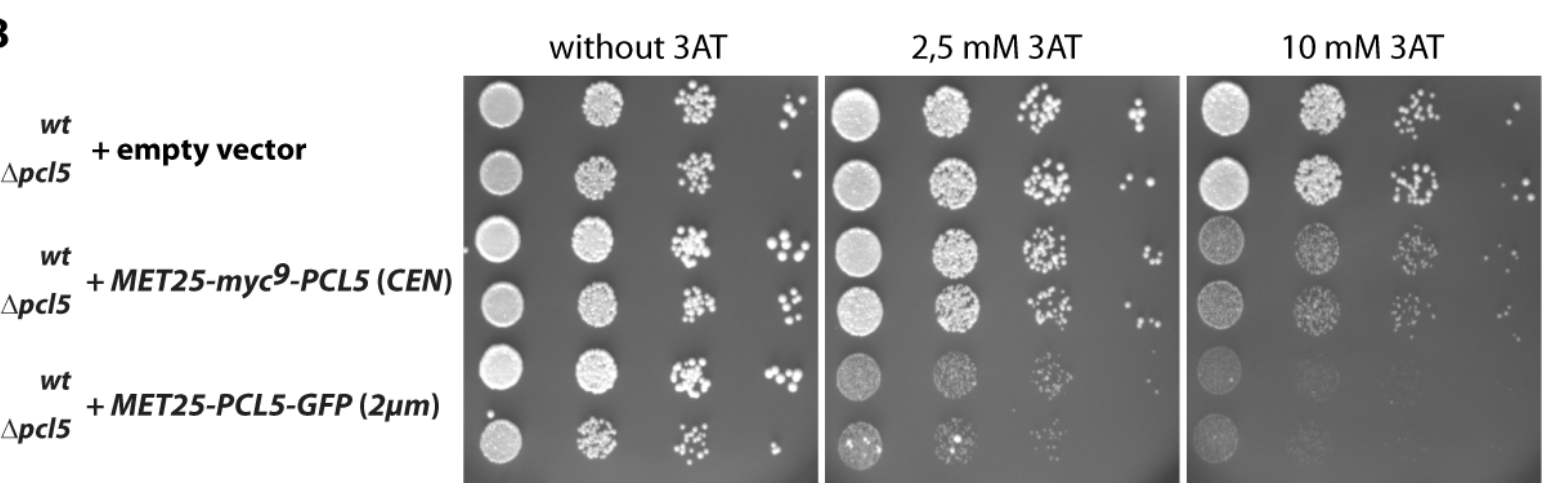

Figure 19: Overexpression of PCL5 impairs proper response to amino acid starvation in S. cerevisiae. (A) Similar levels of Pc15p lead to similar Gcn $4 p$ degradation in sated and starved yeast cells. The S. cerevisiae strain RH3238 (PCL5) was transformed to express $m y c^{3}-G C N 4$ from the highcopy plasmid KB294 under control of the GAL1 promoter together with an empty vector (p415MET25) as control or together with MET25 promoter driven myc ${ }^{9}$-PCL5 (pME3727 on a lowcopy (CEN) plasmid. Additionally, GCN4 was expressed from the GAL1 promoter (KB294) in pcl5 mutant cells (RH3238) together with PCL5-GFP (pME2846) under control of the repressible MET25 promoter on a high-copy $(2 \mu \mathrm{m})$ plasmid. Protein levels of myc ${ }^{3}-\mathrm{Gcn} 4 \mathrm{p}$, myc ${ }^{9}-\mathrm{Pcl} 15 \mathrm{p}$, or Pcl5-GFP, as well as Cdc28p or eIF2p as loading control were determined in sated (+Tryptophan) and amino acid starved (-Tryptophan) cells after the promoter shutoff. Furthermore, phosphorylated eIF2 $\alpha-P p$ confirms amino acid starvation. (B) Overexpression of PCL5 results in sensitivity towards amino acid starvation. Yeast strains RH3237 (PCL5) and RH3238 (pcl5) with empty vectors or expressing myc ${ }^{9}-$ PCL5 (pME3727) from a low-copy (CEN) plasmid or PCL5-GFP (pME2846) from a high-copy $(2 \mu \mathrm{m})$ plasmid under control of the MET25 promoter were spotted in ten-fold dilutions on minimal medium without, with $2,5 \mathrm{mM}, 5 \mathrm{mM}$, and $10 \mathrm{mM} 3 \mathrm{AT}$. Plates were incubated for three days at $30^{\circ} \mathrm{C}$.

Taken together, constitutive overexpression of PCL5 leads to a similar rapid degradation of Gen $4 p$ in sated and starved cells and therefore impairs the stabilization of Gen $4 p$ in amino acid starved cells, which is required to circumvent amino acid starvation in yeast. In addition, the fact that Gen $4 p$ is rapidly degraded under amino acid starvation further support that the 
Pho85p/Pc15p is not disrupted under this condition and corroborate the hypothesis that Gcn $4 p$ stabilization is due to the disappearance of Pc15p in starved cells.

\subsubsection{Overexpression of $P C L 7$ counteracts $P h 085 p / P c l 5 p$ activity}

Besides Pcl5p, the CDK Pho85p is known to interact with at least nine other cyclins (Measday et al., 1997). One of these cyclins is Pcl7p, which was initially shown to be involved in the regulation of glycogen biosynthesis and catabolism but which also participates in the Gcn4p stability regulation (Lee et al., 2000; Bömeke et al., 2006). Furthermore, the cyclin Pcl7p interacts with the CKI Pho81p. Pho81p was suggested to regulate the activity of both complexes, Pho85/Pho80p and Pho85p/Pcl7p, depending on the phosphate availability without loss of interaction. Similarly, independent of the amino acid availability, the stable and predominantly nuclear localized Pcl7p was shown to constitutively interact with Pho85p and Pho81p (Bömeke et al., 2006). Deletion of PCL7 resulted in rapid Gen4p degradation in sated and amino acid starved cells (Bömeke et al., 2006) and therefore this cyclin was required for the stabilization of Gen $4 p$ in starved cells. It was suggested that Pcl7p binds to Pho85p antagonistically to Pc15p and that this competition for Pho85p interaction is the way of Pcl7p function in Gen $4 p$ stability regulation. This idea was furthermore supported by the fact that overexpression of PCL7 resulted in sensitivity to high amounts of $3 \mathrm{AT}$ when cells were grown on solid medium (Bömeke et al., 2006). It was proposed that in sated cells a possible equilibrium of Pho85p/Pcl5p and Pho85p/Pcl7p complexes exists with sufficient amounts of Pho85p/Pcl5p complexes for Gcn $4 p$ degradation, which shifts towards less Pho85p/Pcl5p complexes in amino acid starved cells leading to Gcn4p stabilization. This model is furthermore corroborated by the observation shown above that overexpression of PCL5 from the constitutive MET25 promoter, which results in a shift towards more Pho85p/Pc15p complexes, also leads to a rapid and constitutive Gcn4p degradation as in pcl7 mutant cells. However, one assumption of this model was that the Pho85p/Pcl5p and Pho81p/Pcl5p complexes disrupt under amino acid starvation conditions and that these complexes are replaced by Pho85p/Pcl7p complexes. As shown above, this disruption is not the case but the prediction that under amino acid starvation conditions less Pho85p/Pcl5p complexes are formed still seems likely because it was shown that Pc15p rapidly disappears under these conditions. However, the question arose of how Gen $4 p$ is degraded in amino acid starved pcl7 mutant cells when less Pho85p/Pcl5p complexes are present in the cells. Since previous studies have shown that at least in vitro other Pho85p interacting cyclins were able 
to promote Gen4p phosphorylation, including Pcllp (Meimoun et al., 2000), a possible explanation would be that other Pho85p/cyclin complexes initiate Gen4p degradation under these conditions. Therefore, a yeast pcl7 pcl5 double mutant strain (RH3394) was constructed and the Gcn $4 p$ degradation was analyzed. To test the Gcn $4 p$ degradation directly, these tryptophan auxotrophic pcl7 pcl5 double mutant cells were transformed with GAL1 driven myc ${ }^{3}-G C N 4$ (pME2848) and promoter shutoff experiments were executed as described before. Similar to pcl5 mutant cells of strain RH3238 (Figure 10), myc ${ }^{3}$-Gen4p degradation was abolished in sated and starved pcl7 pcl5 double mutant cells (data not shown). Furthermore, these cells showed a similarly reduced growth like the pcl5 single mutant in the already described GCN4 overexpression toxicity assay (data not shown). This indicates that the described constitutive Gcn $4 p$ degradation in pcl7 deficient yeast cells is still dependent on the cyclin Pc15p as described for PCL7 wild-type cells (Shemer et al., 2002; Bömeke et al., 2006).

If the proposed model described above is right and Pcl7p is required for Gcn $4 p$ stabilization by competitive binding with Pcl5p to Pho85p, a prediction would be that overexpression of PHO85 should also lead to degradation of Gen4p under conditions of amino acid starvation but which had never been tested. Promoter shutoff experiments with tryptophan auxotrophic PHO85 wild-type cells (RH3237) expressing myc ${ }^{3}$-GCN4 (pME2848) either with GST (pYGEX-2T) as control or with GST-PHO85 (pME2866) from the inducible GAL1 promoter were accomplished as described above. Starvation was again induced for 45 minutes in minimal medium lacking tryptophan. Protein levels of myc ${ }^{3}-\mathrm{Gcn} 4 \mathrm{p}$ of two independently performed experiments were analyzed after GAL1 promoter shutoff in sated and amino acid starved cells. Western hybridization revealed that cells overexpressing PHO85 were unable to stabilize Gen4p in response to amino acid starvation. In contrast, cells expressing GST instead of GST-PHO85 and that therefore only contain endogenous PHO85 (Figure 20) showed Gcn $4 p$ stabilization as expected. Additionally, this experiment verifies that Pho85p is a stable protein independent of the amino acid availability since GST-PHO85 is also expressed from the GAL1 promoter and the level of GST-Pho85p was not significantly reduced in sated and starved cells 30 minutes after promoter shutoff (Figure 20). 


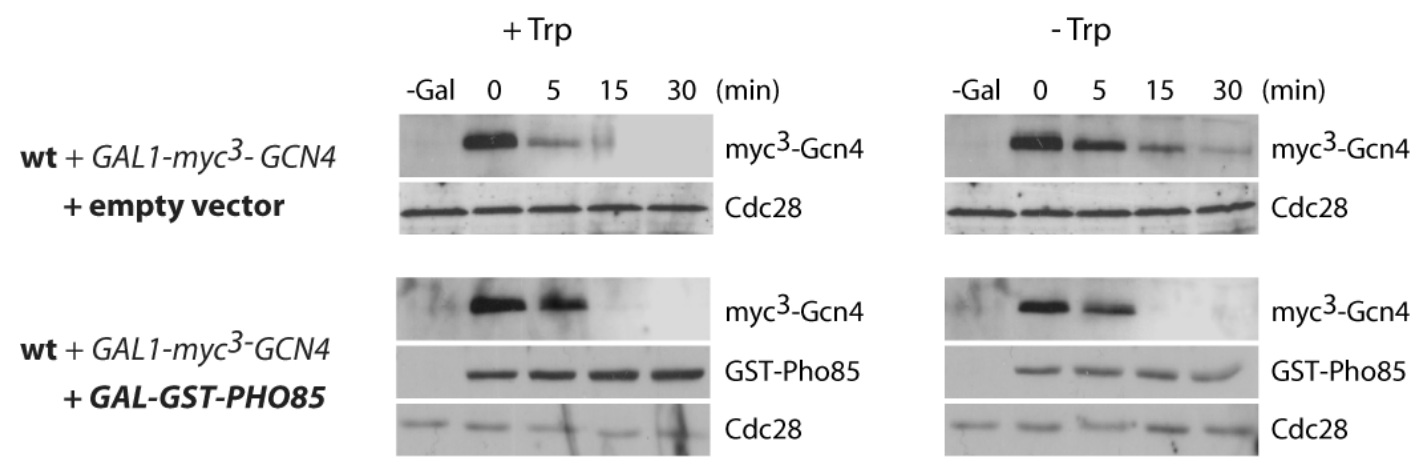

Figure 20: Overexpression of PHO85 impairs Gen4p stabilization in amino acid starved yeast cells. The $S$. cerevisiae strain (RH3238) was transformed to express GAL1 driven myc ${ }^{3}-G C N 4$ on plasmid pME3728 together with GAL1 driven GST-PHO85 (pME2866) or with the empty vector (pYGEX-2T) as control. Cells were grown to early log-phase in selective medium containing glucose and shifted to medium with $2 \%$ galactose to induce GAL1 driven expression. After 3 hours of induction half of the tryptophan auxotrophic cells were transferred to medium lacking tryptophan to induce starvation for 45 minutes prior to promoter shutoff (time point $0 \mathrm{~min}$ ). Protein levels of myc ${ }^{3}-$ Gcn4p, GST-Pho85p and Cdc28p as loading control were analyzed in sated (+Trp) and amino acid starved (-Trp) cells at the indicated time points.

These results suggest that the amount of uncomplexed Pho85p which is able to interact with also unbound Pcl5p, is important for amino acid dependent Gcn $4 p$ stability regulation in yeast. Since both, overexpression of PHO85 and deletion of PCL7 might lead to a shift towards more Pho85p/Pcl5p complexes in the cells resulting in unstable Gcn $4 \mathrm{p}$ in amino acid starved cells, a shift to less Pho85p/Pcl5p complexes in the cell might lead to a stabilization of Gcn $4 p$ independent of the amino acid availability. Therefore, it was analyzed whether overexpression of PCL7 has an influence on Gen4p degradation in sated cells and which has never been shown before. Firstly, promoter shutoff experiments were carried out with sated cells to analyze the Gcn $4 p$ turnover when PCL7 is overexpressed. The methionine prototrophic yeast PCL7 wild-type cells (RH3238) were transformed to express GAL1 driven myc ${ }^{3}-G C N 4$ (pME3728) alone or together with functional GFP-PCL7 (pME2230) from the repressible MET25 promoter. Cells were pre-grown to early exponential phase in the presence of methionine. Cells were harvested and shifted to minimal medium with galactose as carbon source to induce $m y c^{3}-G C N 4$ expression. Half of these cells were incubated in the presence and half in the absence of methionine, conditions that repress or induce expression of MET25 driven GFP-PCL7, respectively. Expression of $m y c^{3}-G C N 4$ was stopped by addition of $2 \%$ glucose to the medium and protein levels were determined by immuno hybridization. Analysis of $\mathrm{myc}^{3}-\mathrm{Gcn} 4 \mathrm{p}$ showed rapid degradation of Gen $4 \mathrm{p}$ in sated methionine prototrophic cells when PCL7 is not or only slightly overexpressed in the presence of methionine (Figure 21). In contrast, Gen $4 p$ was more stable when PCL7 was overexpressed in the absence of 
methionine. Determination of GFP-Pc17p confirmed the overexpression of PCL7 in medium lacking methionine. Moreover, Gcn $4 p$ degradation was unaffected by the lack of methionine without concomitant PCL7 overexpression (Figure 21).

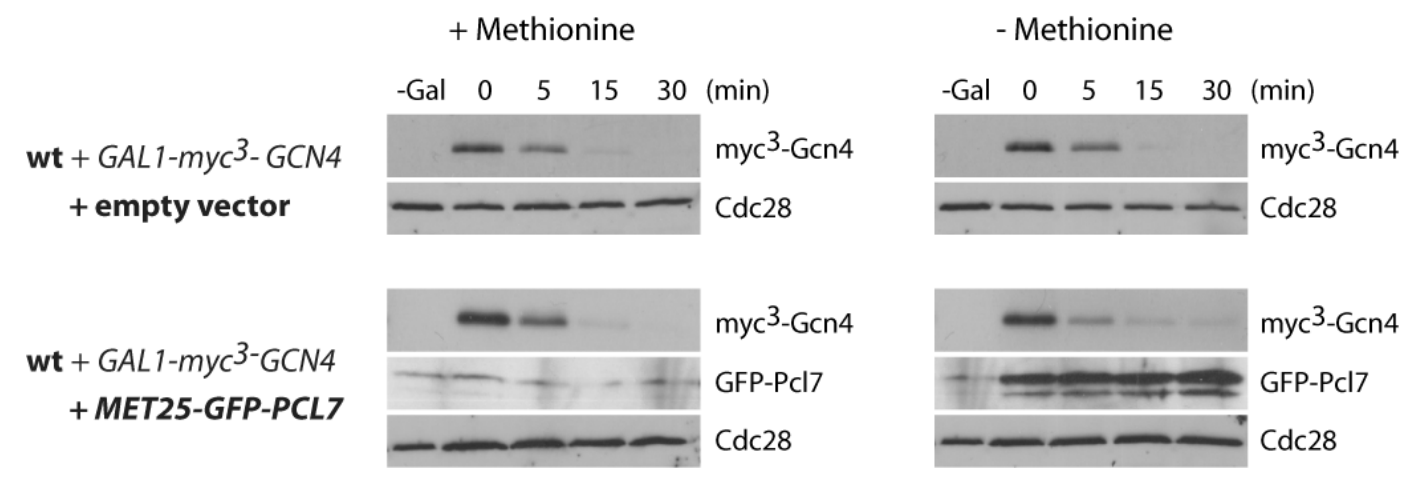

Figure 21: Overexpression of $P C L 7$ leads to less efficient Gcn4p degradation in S. cerevisiae. The methionine prototrophic S. cerevisiae strain (RH3237) was transformed to express GAL1 driven myc ${ }^{3}$-GCN4 (pME3728) alone (empty vector p426MET25) or together with MET25 driven GFP$P C L 7$ (pME2230) in the presence or absence of methionine to repress or induce expression of GFPPcl7p, respectively. Cells were grown to early log-phase in selective medium containing methionine and glucose and shifted to medium with $2 \%$ galactose to induce GAL1 driven GCN4 expression for three hours either in the presence or absence of methionine. Samples were taken at the indicated time after GAL1 promoter shutoff and protein levels of myc ${ }^{3}-G c n 4 p$, GFP-Pcl7p, and Cdc28p as loading control were analyzed by Western hybridization.

These data indicate that high amounts of Pcl7p lead to less efficient Gcn $4 p$ degradation under non-starvation conditions. Therefore, the effect of PCL7 overexpression on GCN4 overexpression toxicity was investigated as already described. Yeast strain RH3237 (PCL5) was transformed with GAL1 driven myc ${ }^{3}$-GCN4 (pME2848) together with functional MET25 driven PCL7 (pME2933) alone, or in addition with MET25-PHO81-GFP (pME2228), or MET25-PCL5-GFP (pME2846), or GAL1-GST-PHO85 (pME2866) instead of an empty vector (p426MET25). As control, yeast strain RH3237 (PCL5) and RH3238 (pcl5) were transformed with GAL1 driven myc ${ }^{3}-G C N 4$ (pME2848) together with the empty vectors (p424MET25 and p426MET25). The GCN4 overexpression toxicity revealed that overexpression of PCL7 led to a slightly reduced growth when GCN4 is expressed on galactose medium (Figure 22). Compared to the growth reduction of pcl5 mutant cells when GCN4 is overexpressed, additional overexpression of PCL7 had just a little effect as demonstrated in Figure 22. Simultaneous overexpression of PHO81 did not alter the reduced growth whereas concomitant overexpression of PCL5 or PHO85 suppressed the reduced growth (Figure 22). 


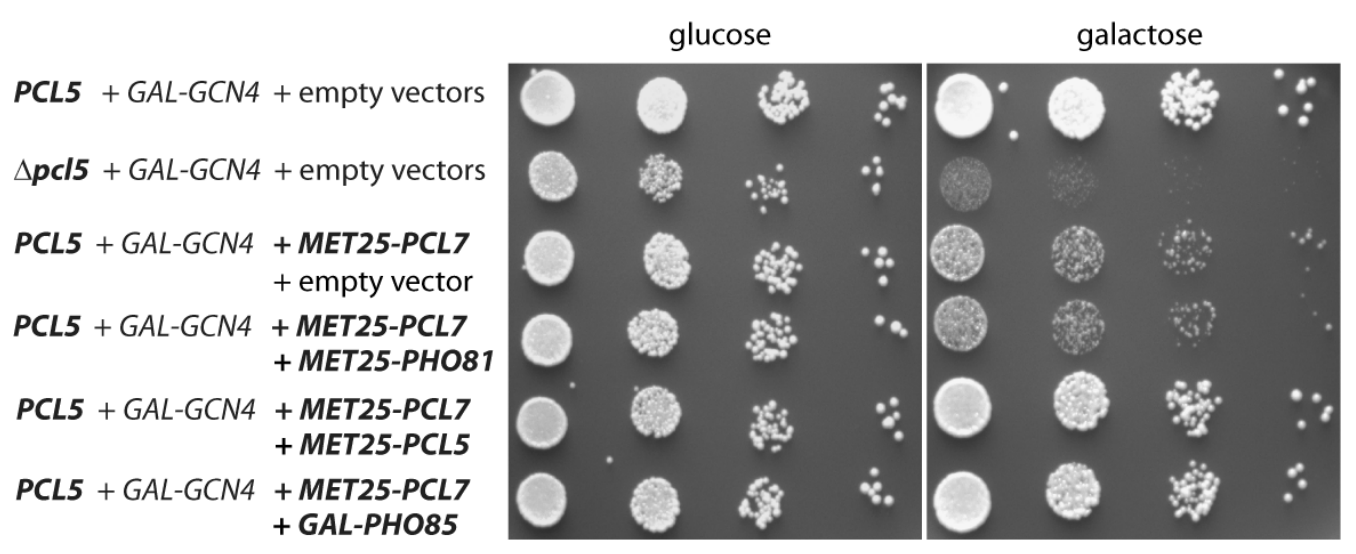

Figure 22: Overexpression of PCL7 induces GCN4 overexpression toxicity in PCL5 cells. Yeast strain RH3237 (PCL5) and RH3238 (pcl5) were transformed to express GAL1 driven GCN4 (pME2848) together with empty vectors (p424MET25 and p426MET25) as controls. Furthermore, wild-type cells (RH3237) were transformed to express GAL1-GCN4 together with MET25 driven PCL7 (pME2933) and an empty vector (p426MET25), or with PCL7 (pME2933) in conjunction with MET25 driven PHO81-GFP (pME2228) or PCL5-GFP (pME2846), or GAL1 driven GST-PHO85 (pME2866). Transformants were spotted in ten-fold dilutions on appropriate minimal medium with either glucose or galactose as carbon source to induce GAL1 dependent GCN4 expression. Plates were incubated for 3 days at $30^{\circ} \mathrm{C}$.

Taken together, this suggests that high levels of Pcl7p lead to GCN4 overexpression toxicity in wild-type yeast cells due to the above described slight stabilization of Gen4p in sated cells when PCL7 is overexpressed. Moreover, previous studies have shown that overexpression of $P C L 7$ resulted in strong stabilization of Gen4p under amino acid starvation conditions and in an increased sensitivity towards high concentration of the histidine analogue 3AT (Bömeke et al., 2006). The data presented above are consistent with these findings but contradictory to the also described slightly increased sensitivity of pcl7 mutant cells (Bömeke et al., 2006). Therefore, the sensitivity to low and high amounts of 3AT of the yeast strains RH3237 (wildtype), RH3241 (pho81), RH3255 (pcl7), and RH3238 (pcl5) transformed with empty vectors only (p424MET25 and p426MET25) was compared to wild-type cells (RH3237) and pho81 mutant cells (RH3241) each transformed to express MET25 driven PCL7 (pME2933). Furthermore, wild-type cells (RH3237) transformed with MET25-PCL7 (pME2933) together with MET25-PHO85 (pME3431) were used for the drop dilution assay shown in Figure 23. Overexpression of PCL7 resulted in an increased sensitivity to high amounts of 3AT as previously described (Bömeke et al., 2006). Consistently, pcl5 mutant cells showed a similar sensitivity to $100 \mathrm{mM}$ 3AT (Figure. 23). In contrast, deletion of pcl7 did not affect 3AT sensitivity compared to PCL7 wild-type cells. Similarly, pho81 mutant cells were not affected (Figure 23). Deletion of PHO81 might lead to a very weak increased PCL7 overexpressioninduced hypersensitivity compared to PHO81 wild-type cells (Figure 23). Furthermore, 
simultaneous overexpression of PCL7 and PHO81 but also overexpression of PHO81 alone did not alter the resistance to 3AT (data not shown). Consistent with these and the above described findings, PCL7 overexpression-induced 3AT sensitivity was suppressed by concomitant overexpression of PHO85 (Figure 23). These data support the assumption that the hypersensitivity to high amounts of 3AT might be caused by toxic Gen4p levels which are only achieved because of the increased Gcn $4 p$ stability. This in turn is due to the PCL7 overexpression.

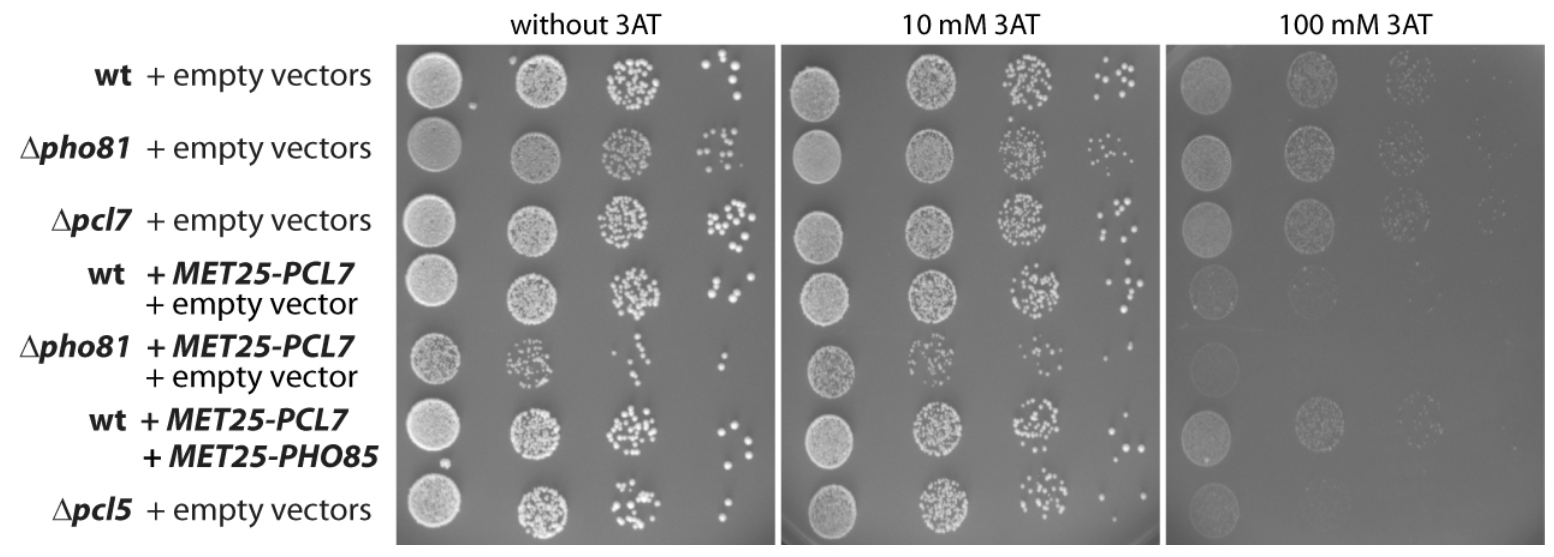

Figure 23: Concomitant overexpression of $P H O 85$ suppresses $P C L 7$ overexpression-induced hypersensitivity to 3AT. The histidine prototrophic yeast strains RH3237 (wild-type), RH3241 (pho81), RH3255 (pcl7), and RH3238 (pcl5) transformed with empty vectors only (p424MET25 and p426MET25), and RH3237 (wild-type) and RH3241 (pho81) transformed to express PCL7 (pME2933) from the MET25 promoter together with the empty vector p426MET25, as well as wildtype cells (RH3237) transformed to express MET25-PCL7 (pME2933) together with MET25 driven PHO85 (pME3431) were dropped in ten-fold dilutions on minimal medium without, with $10 \mathrm{mM}$, or with $100 \mathrm{mM} 3 \mathrm{AT}$. Plates were incubated at $30^{\circ} \mathrm{C}$ for 3 days.

In summary, the presented data show that high levels of Pcl7p diminish Gen $4 p$ degradation leading to slight stabilization of Gcn4p even in sated cells. Furthermore, PCL7 overexpression impairs a proper response to amino acid starvation induced by high amounts of 3AT. Since overexpression of PHO85 or PCL5 suppresses the effects of high amounts of Pcl7p the data suggest that these findings might be due to an imbalance of Pcl7p and Pcl5p levels in competition to bind Pho85p. Therefore, overexpression of PCL7 leads to a shift towards more Pho85p/Pcl7p complexes but less Pho85p/Pcl5p complexes. Additionally, the lack of PHO81 might increase this PCL7 overexpression effect. 


\section{Discussion}

In this study, the nuclear import of the cyclin Pc15p was characterized in more detail showing that the importin Kap95p is indeed mainly responsible for the Pcl5p nuclear localization. Furthermore, a motif similar to a classical NLS is required that the C-terminal 49 amino acids of Pcl5p function as non-canonical NLS.

Interaction studies of Pcl5p and the CDK Pho85p as well as Pcl5p and the CKI Pho81p combined with Pc15p and Pcl7p overexpression studies support the idea that the amount of Pc15p that competes for binding to Pho85p with other cyclins in the cell is the major regulatory mechanism in the control of Gen $4 p$ stability.

\subsection{Pcl5p nuclear import requires a C-terminal NLS and is mediated by the $\beta$ importin Kap95p}

The cyclin Pc15p in association with the CDK Pho85p is specifically required to initiate rapid Gen $4 p$ degradation under conditions of nutrient saturation when Gen $4 p$ is not needed (Shemer et al., 2002). Since the regulated and efficient turnover of the transcription factor Gcn $4 p$ is an exclusively nuclear localized process (Pries et al., 2002), the involved proteins have to be transported into the nucleus to fulfill their function. Accordingly, nuclear translocation of Pcl5p takes place under amino acid rich conditions when Gen $4 p$ is rapidly degraded. Furthermore, this nuclear transport was shown to be independent of the amino acid availability and Pcl5p is also a predominantly nuclear localized protein when the cells are starved for amino acids, conditions that stabilize Gcn4p. In addition, the Pc15p nuclear localization does not require the interacting proteins Pho85p, Pho81p, or Gen $4 p$, but was described to be necessary for Gcn4p turnover (Bömeke, 2006). It has been shown that the carboxyl terminus of the cyclin extending from amino acid 181 to 229 localizes GFP to the nucleus and is sufficient to mediate Pc15p nuclear import (Bömeke, 2006). It was furthermore suggested that the $\beta$ importin Kap95p participates in the nuclear translocation of Pc15p since kap95 mutant cells showed an increased amount of Pc15p-GFP in the cytoplasm. In contrast, deletion of other $\beta$ importin-encoding genes had no obvious effect on Pcl5p localization (Bömeke, 2006).

In this work, Kap95p has been shown to import Pcl5p into the nucleus by a physical interaction. Overexpression of KAP95 restored the nuclear localization of Pcl5-GFP in 
temperature sensitive kap95 mutant cells at the restrictive temperatures of $30^{\circ} \mathrm{C}$ and $37^{\circ} \mathrm{C}$ (Figure 11). However, there was still a subpopulation of approximately $30 \%$ of the cells showing cytoplasmic Pcl5-GFP aggregates similar to the situation in kap95 cells. Furthermore, in vivo coprecipitation assays demonstrated that Kap95p and Pcl5p interact. This interaction was not due to the overexpression of KAP95 or PCL5 since Pcl5p did not interact with two other $\beta$ importins, Kap104p and Pse1p, tested under similar conditions (Figure 11). The fact that there was still a small amount of Pcl5-GFP detectable in the nucleus in kap95 impaired cells suggests that other importins are involved in the import of Pcl5p into the nucleus or can at least partially undertake Kap95p function. Consistently, there are many examples in the literature for a substrate transported by more than one importin with different efficiency. In mammalian cells at least four $\beta$ importins are able to transport ribosomal proteins into the nucleus (Jäkel and Görlich, 1998). Furthermore, the histones H2A and H2B in S. cerevisiae were shown to directly interact with three different karyopherins including Kap95p. In addition, all of these three importins are able to mediate the nuclear transport of H2A and H2B (Mosammaparast et al., 2001). Gcn4p in budding yeast exemplifies another protein that is transported by different importins. The nuclear transport of Gcn $4 p$ depends on two NLS. While NLS2 mediated transport specifically requires the $\alpha$ importin Srp1p and the $\beta$ importin Kap95p, the NLS1 dependent import is unspecifically mediated by several karyopherins (Pries et al., 2004). In case of Pcl5p, it is unlikely that Pse1p or Kap104p transport Pc15p into the nucleus because no interaction was observed in coprecipitation assays but the remaining seven $\beta$ importins can not be excluded so far. To further analyze whether other importins might also participate in Pcl5p nuclear import, the localization of Pcl5p in kap95 mutant cells additionally impaired in other importin genes have to be characterized.

Moreover, Kap95p has been shown to be involved in the classical transport mechanisms of many proteins in complex with Srplp, the only known $\alpha$ importin in budding yeast. Most proteins that were shown to be transported via $\alpha / \beta$ importin complexes contain a classical NLS. The C-terminal part of Pcl5p contains a motif between amino acids 207-215 (PVKRPRESD) that resembles the classical NLS motif of the human c-myc protein (PAAKRVKLD), in which a proline and an aspartic acid residue are at the boundary of the basic cluster (Makkerh et al., 1996). Interestingly, the motif of Pcl5p does not function as classical NLS since it was shown to be insufficient to direct the exclusively cytoplasmic protein Aro7p in a fusion with GFP or GFP alone into the yeast nucleus (Bömeke, 2006) but it was recently demonstrated that importin $\alpha$ can bind more variable NLS patterns than previously thought including non-canonical NLSs (Kosugi et al., 2009). Therefore, it cannot 
be excluded that Srp1p also participates in Pcl5p nuclear transport via complex formation with Kap95p. To discriminate between the classical Srp1p-dependent or a non-classical Srp1p-independent mechanism of Pc15p nuclear import, the localization of Pcl5p should be investigated in cells impaired in Srp1p function. Nevertheless, the efficient transport of Pcl5p into the nucleus of yeast cells is predominantly mediated by the $\beta$ importin Kap95p by a physical interaction.

In order to further characterize the putative NLS motif in the C-terminal domain of Pc15p, the influence of its mutation on Pcl5p localization was analyzed revealing that this motif is required for the $\mathrm{C}$-terminal domain-dependent nuclear import. The mutagenesis of the basic residues of this cluster to alanine results in cytoplasmic retention of the C-terminus of Pcl5p fused to GFP and leads to an increased cytoplasmic localization of full-length Pcl5-GFP (Figure 8). Furthermore, Pcl5p nuclear localization was shown to be a prerequisite for the rapid Gcn4p degradation (Bömeke, 2006). Consistently, impairment of Pcl5p nuclear localization due to the mutated motif does also affect Gen $4 p$ degradation (Figure 9 and 10).

Since the putative NLS motif is necessary but not sufficient to mediate the transport of fusion proteins into the nucleus additional sequences at least of the $\mathrm{C}$-terminal domain seem to be required for the proper transport of Pc15p into the nucleus. This is further supported by the fact that mutation of the NLS motif does not prevent the interaction of Pcl5p with the importin Kap95p. In addition, the C-terminal domain of Pcl5p alone including the nonmutated motif does not interact with Kap95p under the same conditions (Figure 11) but it is quite possible that the N-terminal fusion of the nine-fold myc epitope tag impairs the binding to Kap95p.

However, a classical NLS is not mandatory for nuclear transport and NLSs have been shown to be quite diverse and sometimes a very long segment of a protein is identified as its NLS. It was shown that the RNA binding protein Lhp1p in S. cerevisiae is brought into the nucleus by a 113 amino acid domain whose three-dimensional structure is important for its function (Rosenblum et al., 1998). Another example is given by the lymphoid specific factor RAG2 which is essential for diversification of antigen receptors on $\mathrm{B}$ and $\mathrm{T}$ lymphocytes. This protein contains a classical NLS-related motif (PAKKSFLRRLFD) which is necessary but not sufficient for its translocation into the nucleus similar to the motif in Pcl5p. To localize RAG2 to the nucleus, a C-terminal 37 amino acid minimal domain is required which includes its classical NLS related motif (Corneo et al., 2002). In the present case of Pcl5p, the Cterminal 49 amino acid residues (aa 181-229) containing the necessary motif are sufficient to direct GFP into the nucleus and therefore represent the non-canonical NLS. 
Besides this non-canonical NLS, other parts of Pcl5p were suggested to contribute to the nuclear localization of this cyclin since the middle part of Pcl5p (amino acid residues 61-180) containing the cyclin box domain, fused to GFP showed a cytoplasmic dot-like aggregate phenotype and was unable to mediate Gcn4p degradation whereas Pcl5aa1-180-GFP was nuclear localized with additional dot like structures in the cell and mediated the Gcn $4 p$ degradation (Bömeke, 2006). According to this, not only Pcl5aa1-180p without the entire C-terminus but also Pc15aa1-229***-GFP with the mutated motif in the C-terminal domain and which therefore lacks any strong NLS was still nuclear localized (Figure 8). Furthermore, both fusion proteins show some Gcn4p degradation when these constructs are overexpressed (Figure 9 and 10). In addition, Pcl5aa1-180p lacking the C-terminal NLS showed some nuclear enrichment even when expressed from a low copy plasmid (Figure 12) and was still able to mediate Gen4p degradation (Figure 13). The N-terminal domain of Pcl5p contains eight $\mathrm{R} / \mathrm{K}$ residues including one RKK stretch that may act as an ancillary nuclear localization motif similar to the substrate Gen4p, which was shown to have two NLSs. NLS2 is the specific and essential nuclear transport signal, whereas NLS1 contributes only as auxiliary signal (Pries et al., 2004). However, since the N-terminal part of Pcl5p (amino acid residues 1 to 95) without the entire cyclin box domain (amino acids 80 to 180) was shown to result in plasma membrane associated localization when fused to GFP (Bömeke, 2006) this putative auxiliary signal requires additional sequences presumably within the cyclin box domain. Nevertheless, the fact that mutation of the basic cluster within the C-terminus of the cyclin did not abolish the interaction of Pc15p with the karyopherin Kap95p further supports the idea of an ancillary signal involved in the proper nuclear import of the cyclin (Figure 11).

Furthermore, bimolecular fluorescence complementation analysis of Pcl5p and Pho85p revealed that both proteins predominantly interact with each other in the nucleus but also in the cytoplasm (data not shown). Another but highly speculative explanation for the residual import of the constructs described above is that they are brought into the nucleus by piggy backing with Pho85p or another known interacting protein. Such a transport mechanism is normally not required due to the C-terminal NLS and would therefore be masked in cells lacking Pho85p as desribed by Bömeke (2006). However, the residual import capacity remains a future task.

The two previous and partially connected studies using N- and C-terminally truncated Pcl5p versions as well as hybrids of the N-terminal and C-terminal domain of the Pho85p cyclin Pho80p fused to the cyclin box domain of Pcl5p (Pho80-Pc15-Pho80) nicely demonstrated that the cyclin box domain of Pcl5p is responsible for the substrate specificity towards Gen $4 p$ 
(Bömeke et al., 2006; Aviram et al., 2008). Since a hybrid of the N-terminal domain of Pho80p with the cyclin box domain of Pcl5p fused to GFP (Pho80-Pcl5-GFP) was a predominantly nuclear localized protein, it was suggested that the cyclin Pho80p is a nuclear protein dependent on its N-terminal domain (Bömeke, 2006). As demonstrated in this study, the Pho80-Pcl5-GFP fusion protein is transported into the nucleus even when this construct is expressed from the weak PCL5 promoter (Figure 12). Furthermore, this construct suppresses GCN4 overexpression toxicity and Gen4p degradation similar to full-length Pcl5p (Figure 13). Despite the fact that the Pho80-Pcl5-GFP fusion protein should appear much more stable than Pcl5p due to the lack of the Pcl5p N- and C-terminal domains that contain the Pcl5p destruction signals (Aviram et al., 2008) similar to the Pho80-Pcl5-Pho80 hybrid (Figure 16), this study excludes the possibility that the nuclear import is only due to the overexpression conditions used before. To verify that the N-terminal domain of Pho80p alone is sufficient to direct the Pcl5p cyclin box domain or Pho80p in the nucleus, the localization of this domain alone fused to GFP needs to be analyzed. So far it can not be ruled out that additional parts might contribute to the proper nuclear localization since this domain does not contain any obvious NLS.

In addition to the described functions of the $\mathrm{N}$ - and C-terminal parts of Pho80p and Pc15p in the nuclear localization of the cyclin box domain of Pcl5p, these sequences seem to further contribute to the proper function of Pcl5p. This is supported by the fact that the middle part of Pcl5p (amino acids 61-180) that contains the cyclin box domain, responsible for substrate specificity towards Gen4p, fused to a classical NLS motif of the simian virus 40 large tumor antigen is visible as aggregates in the cell and does not promote Gcn4p turnover similar to the middle part alone fused to GFP. In contrast, constructs that contained N- or C-terminal parts in addition to the cyclin box domain of Pc15p displayed only sometimes aggregates in the cell in addition to their predominantly cytoplasmic or nuclear localization. Furthermore, this was independent of the nuclear import since the predominantly cytoplasmic localized Pcl5aa61-229*** fused to GFP which is unable to mediate Gen4p degradation as well as the predominantly nuclear fusion proteins Pcl5aa1-180-GFP and Pcl5aa61-229-GFP which are both able to mediate Gcn $4 p$ turnover showed these dot-like aggregates in the cell with similar frequency. Moreover, the nuclear localized Pho80-Pc15-GFP fusion protein that contains the N-terminal domain of the cyclin Pho80p does not show the cytoplasmic aggregate phenotype (Figure 12) whereas the cyclin box domain of Pcl5p alone fused to GFP (amino acids 79 to 178) shows aggregates covering the whole cell similar to amino acids 61 to 180 of Pcl5p. 
Therefore, the $\mathrm{N}$ - or C-terminal domains of Pcl5p might participate to the function of the cyclin in different ways possibly by maintenance of the structural integrity of the cyclin box domain, at the level of substrate recognition or Pho85p interaction, or by a residual nuclear import capacity.

In conclusion, the domains of the Pho85p cyclins Pcl5p and Pho80p which are responsible for nuclear import were confirmed as distinct domains that can be exchanged. Pcl5p nuclear transport depends on the C-terminal domain of amino acids 181 to 229 that functions as a non-classical NLS including a necessary motif between amino acids 207-215 (PVKRPRESD) and is mediated by the $\beta$ importin Kap95p by a physical interaction (Figure 24).

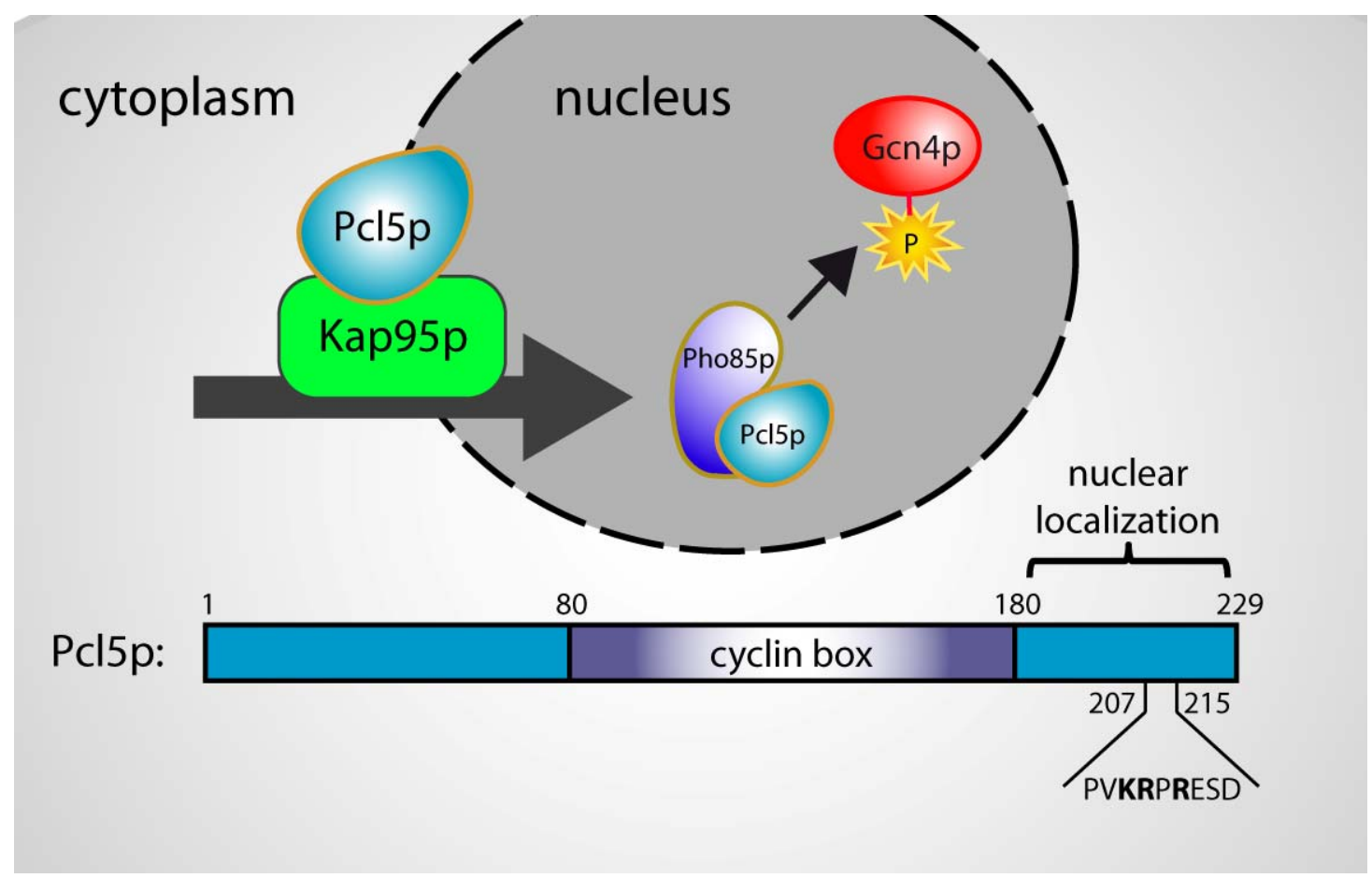

Figure 24: Proposed model of the Pcl5p nuclear import. The constitutive nuclear import of Pcl5p is mediated by the $\beta$ importin Kap95p by a physical interaction. The nuclear translocation of the cyclin Pcl5p depends on its C-terminal domain (aa181-229) that represents a non-classical NLS containing a necessary motif (aa207-215) which is similar to the NLS of the human c-myc protein. Furthermore, the nuclear localization of Pcl5p is independent of the known interacting proteins Pho85p, Pho81p or Gcn $4 p$ but is a prerequisite that the kinase/cyclin complex Pho85p/Pcl5p phosphorylates Gcn $4 p$ which leads to the rapid degradation of this transcription factor via the ubiquitin-proteasome pathway. 


\subsection{The interaction of Pho85p and Pho81p with Pcl5p and the role of Pcl7p in the Gen4p stability regulation}

The rapid turnover of the transcription factor Gcn $4 p$ is an exclusively nuclear process and all known players involved in the Gcn $4 p$ stability regulation are predominantly nuclear localized proteins, as mentioned above (Pries et al., 2002; Bömeke, 2006). In sated yeast cells the Gcn $4 p$ degradation is initiated by the CDK Pho85p in association with its highly instable cyclin Pc15p (Shemer et al., 2002; Aviram et al., 2008). This complex phosphorylates Gcn4p at Thr165 residue which marks the protein for its degradation by the ubiquitin-proteasome pathway, whereas the transcription factor is stabilized under amino acid starvation conditions when it is needed leading to its increased half-life from just a few to up to 20 minutes.

This stabilization was shown to require the cyclin-dependent kinase inhibitor Pho81p as well as a further Pho85p cyclin, Pc17p. In contrast to Pcl7p that interacts with Pho85p and Pho81p independently of the amino acid supply of the cell, the interaction of Pcl5p with Pho85p and with Pho81p is disrupted in amino acid starved cells. It was suggested that this dissociation of the Pho85p/Pcl5p complex initiates the stabilization of Gcn4p (Bömeke et al., 2006). In addition, the cyclin Pcl5p was shown to rapidly disappear from the cells when amino acids become limiting which was suggested to be due to the overall reduced rate of protein synthesis combined with the rapid turnover of Pcl5p (Shemer et al., 2002). Moreover, it was recently demonstrated that the constitutive and rapid turnover of Pcl5p is required for the Gcn4p mediated response to amino acid depletion (Aviram et al., 2008).

\subsubsection{Five amino acids in Pcl5p putatively mediate the interaction with Pho85p and Pho81p}

So far, not much is known about the molecular interactions involved in binding of Pcl5p to Pho85p and Pho81p. The recently described crystal structure of the Pho85p/Pho80p complex shows that five amino acid residues of the cyclin Pho80p (Val129, Leu163, Tyr168, Iso170, and Iso171) bind to three amino acids of the PSTAIRE helix in Pho85p (Iso51, Iso54, and Iso171). The Pho80p amino acid residues involved in the binding are located in the core helices and the connecting loops of the cyclin box domain as well as in the loop C-terminally to the core helix $\alpha 5$ (Figure 25A and B). The Pcl5p cyclin box domain was recently shown by Aviram et al. (2008) to be responsible for the substrate specificity towards Gen4p and, based 
on the Pho85p/Pho80p structure, predicted to form a similar core 5-helix structure like Pho80p. Sequence alignment shows that the amino acid residues Leu137, Leu172, Tyr176, Leu178, and Ala179 are at the corresponding positions in Pcl5p (Figure 25C).

A

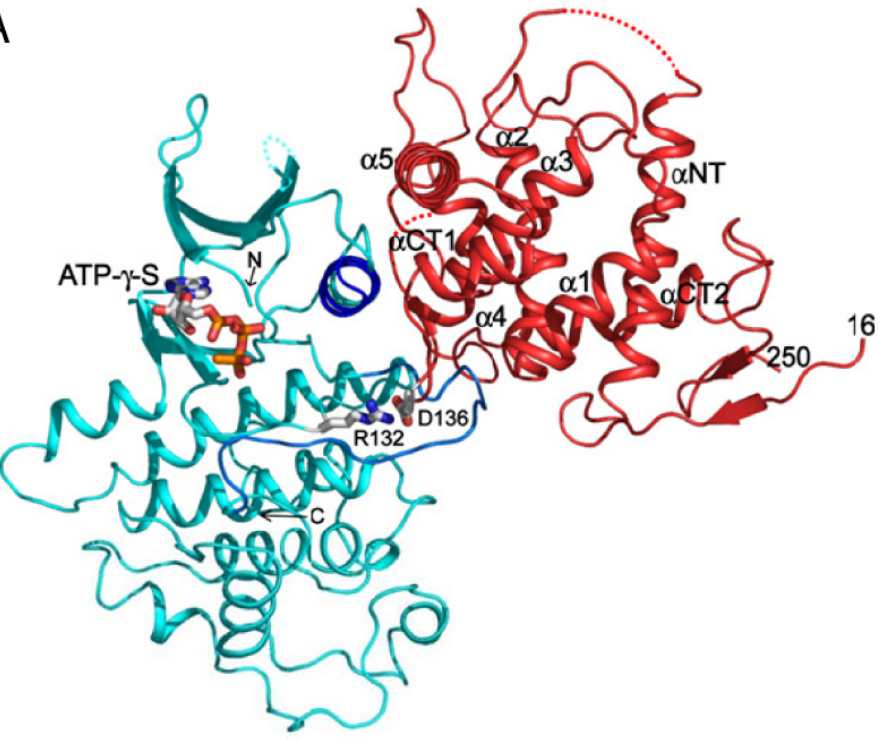

B

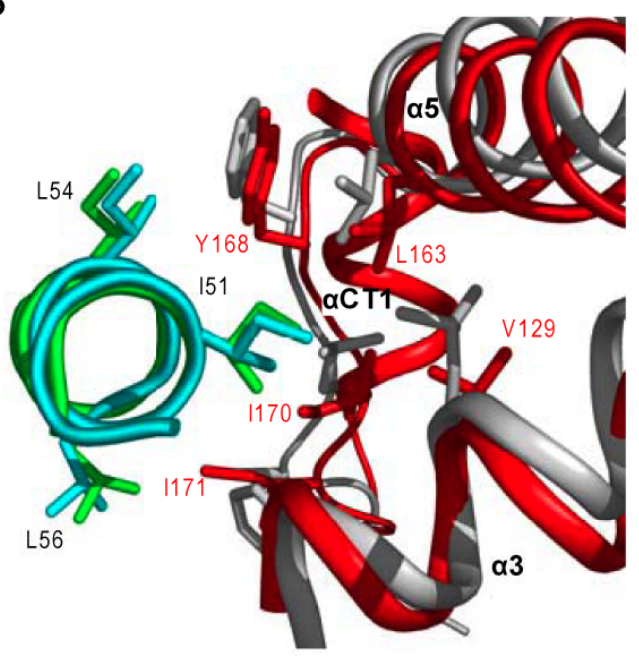

C

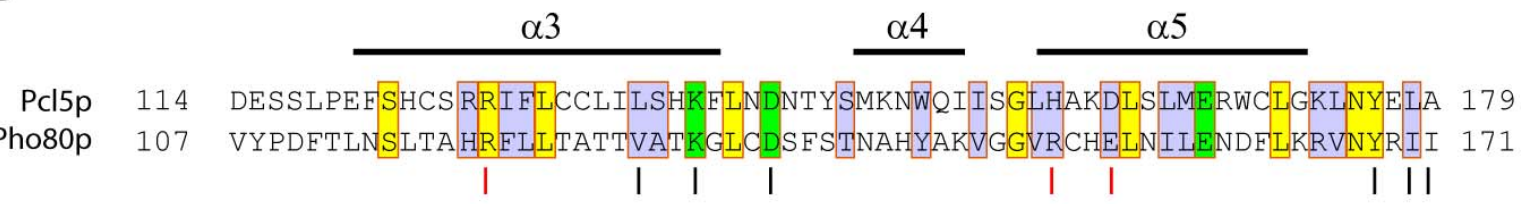

Figure 25: Structure of the Pho85p/Pho80p complex in S. cerevisiae (Huang et al., 2007b) and sequence alignment of the middle parts of the Pho85p interacting cyclins Pcl5p and Pho80p.

(A) Ribbon backbone trace of the crystal structure of the Pho85p/Pho80p complex with bound ATP analog. Pho85p is shown in cyan and Pho80p in red. The Pho85p PSTAIRE helix (blue) binds to Pho80p amino acids of the cyclin box domain that are located within the core helices $\alpha 3$ and $\alpha 5$, and in the connecting loops. Further interacting sites are located in the C-terminal loop next to the core helix $\alpha 5$. Additional helices of Pho80p are located N-terminally $(\alpha \mathrm{NT})$ and C-terminally $(\alpha \mathrm{C} 1$ and $\alpha \mathrm{C} 2)$ to the cyclin box. The amino acid residue R132 of Pho85p and D136 of Pho80p form an activating salt bridge. (B) Hydrophobic interactions of three aliphatic amino acid residues (black) of the Pho85p PSTAIRE helix (blue) with five amino acid residues (red) of the cyclin Pho80p in comparison to the interaction of CDK2 (green) with cyclin A (grey). (C) The sequence of Pcl5p amino acids 114 to 179 and Pho80p amino acids 107 to 171 of S. cerevisiae is shown. The position of the helices of the cyclin box domain of Pho80p $(\alpha 3-\alpha 5)$ of this region are based on the Pho80p structure (Huang et al., 2007b). Green boxes depict identical amino acids in all ten Pho85p cyclins. Identical amino acids of Pcl5p and Pho80p are marked by yellow boxes whereas related amino acids are highlighted by blue boxes. Amino acids of Pho80p that were shown to interact with Pho85p are marked by a black line and amino acids that are suggested to form interaction sites with the CKI Pho81p are depicted by red lines (based on Huang et al., 2007). 
Furthermore, Asp136 of Pho80p in the so-called D-loop between helices $\alpha 4$ and $\alpha 5$ forms a salt-link with Arg132 on the C lobe of Pho85p that makes T-loop phosphorylation of Pho85p no longer necessary for full kinase activity. This aspartate residue is conserved in all Pho85p cyclins and located at position 144 in Pcl5p (Figure 25C). Therefore, the predicted sites in Pcl5p that bind Pho85p are predominantly located in the cyclin box domain. In addition, the amino acid residues Arg121, Arg151, and Glu154 within the cyclin box domain of Pho80p participate in the interaction with the CKI Pho81p. The corresponding Pcl5p amino acid residues are Arg129, His159, and Asp162 (Figure 25C). These predictions fit together well with the interaction studies of this work. Different constructs containing the cyclin box domain of Pcl5p with additional N- or C-terminal sequences of either Pcl5p or Pho80p interacted with Pho85p and Pho81p without any detected differences. Since Pcl5aa1-180 and Pcl5aa61-229 interacted like full-length Pcl5p (Figure 15 and 17, respectively), neither the Nnor the C-terminus of Pcl5p is exclusively necessary for the assembly of the complexes.

Besides mediating the substrate specificity, binding of the kinase or the inhibitor seems to be another feature that is mainly dependent on the cyclin box domain of Pcl5p. However, it remains unclear whether the cyclin box domain alone is able to mediate the interaction to Pho85p or Pho81p. Since the middle part alone (amino acid residues 61-180 and 79-178) or in fusion with a classical NLS was only visible as aggregates in the cell and did not mediate Gcn $4 p$ degradation, it is very likely that additional sequences indirectly participate in binding by maintenance of the structure of the protein rather than being directly responsible for the interaction. Nevertheless there is still the possibility that additional sequences are directly involved in the binding. Furthermore, it is unknown whether the predicted amino acid residues form the interaction or whether other residues are responsible. Mutation of these amino acids, either alone or in combination, and further interaction studies could give new insights.

\subsubsection{Pho85p/Pcl5p and Pho81 $/$ /Pcl5p complex formation is independent of the availability of amino acids}

Previous studies suggested that amino acid starvation induced Gen $4 p$ stabilization is initiated by disruption of the Pho85p/Pcl5p complexes by an unknown mechanism that possibly involves posttranslational modifications presumably of the cyclin. However, 2-dimensional gel electrophoresis of protein extracts from trypthophan auxotrophic cells expressing a myc ${ }^{9}-$ 
PCL5 variant and which were either grown in the presence of amino acids or were starved for tryptophan did not yield reliable and reproducible results in the subsequent immunodetection of the myc epitope tagged Pcl5p (data not shown). Therefore, it cannot be excluded nor demonstrated that Pcl5p is differentially modified in sated compared to starved cells.

However, since substrate specificity and subcellular localization are mediated by distinct domains of the cyclin, it was assumed that disassembly of the complex is also dependent on a specific domain of the cyclin. Due to the fact that a Pho80-Pc15-Pho80p hybrid is able to initiate Gen $4 p$ degradation, interaction studies between this hybrid and Pho85p and Pho81p were performed to elucidate the role of the Pcl5p cyclin box domain in disassembly of the complexes in amino acid starved cells. Interestingly, the interaction of the Pho80-Pc15-Pho80p hybrid with the kinase and the CDK inhibitor was independent of the amino acid availability (Figure 14) suggesting that the previously described dissociation of Pho85p/Pcl5p complex is not dependent on the cyclin box domain of Pcl5p. Therefore, N- or C-terminally truncated versions of Pc15p were analyzed concerning their complex formation with Pho85p and Pho81p. The lack of either the N-or the C-terminus seemed to inhibit the dissociation of the complexes (Figure 15) but the studies furthermore revealed that the amount of coprecipitated cyclin is intensively dependent on the amount of cyclin in the initial extract. Moreover, it became clear that the level of Pcl5p in amino acid starved cells was much less than in sated cells and this effect was much more pronounced than one would expect. Therefore, the level of Pcl5p in the initial extract from starved and sated cells was quantified and the amount of extract in the coprecipitation assay was normalized accordingly. In contrast to previous studies, Pc15p interacted with the CDK Pho85p and the CKI Pho81p independent of the availability of the amino acid tryptophan (Figure 17). Consistently, constitutive overexpression of PCL5 resulted in rapid degradation of Gen $4 p$ in sated and starved cells (Figure 19A). Therefore, disassembly of the Pho85p/Pcl5p complexes seems not to be the regulatory mechanism in the amino acid dependent stability control of Gcn $4 p$, at least in response to tryptophan starvation. So far, only tryptophan depletion was used to induce amino acid starvation in the coprecipitation assays as well as in the performed Gcn $4 p$ stability assays and therefore it remains for the future to test whether the described findings are only subject to tryptophan starvation or if similar results will be obtained when for example leucine auxotrophic cells are starved for leucine. In contrast, it can be excluded that the results are due to a loss of the auxotrophic marker since tryptophan starvation resulted in significant growth arrest of the cells leading to less harvested cells. 
However, the fact that overexpression of PCL5 leads to hypersensitivity to 3AT which is more pronounced when PCL5 is expressed from a high copy plasmid compared to a low copy vector (Figure 19B) corroborates the results. In addition, the fact that the Pcl5p and Pho85p interaction is not disrupted in amino acid starved cells is in good agreement to the recently reported data of Aviram et al. (2008) It was shown that autophosphorylation of threonine 32 in the Pc15p N-terminal part by the interacting Pho85p molecule leads to the rapid degradation of the cyclin which is essential for the response to amino acid limitation and leads to an increased sensitivity to 3AT as well as impaired Gen4p stabilization in starved cells when the stabilized Pc15p is expressed from the weak PCL5 promoter (Aviram et al., 2008). Furthermore, a C-terminal destruction signal exists which is independent of Pho85p but requires a free $\mathrm{C}$-terminus and is only involved in the degradation of the cyclin when PCL5 is overexpressed (Aviram et al., 2008). In addition to the different copy numbers of the vectors used for PCL5 overexpression, the stability of the encoded PCL5 versions might further be responsible for the increased sensitivity to $3 \mathrm{AT}$ (Figure 19B). In the N-terminally myc ${ }^{9}$ tagged version of Pc15p on the low copy plasmid the C-terminal destruction signal is not blocked whereas the GFP tag at the C-terminus of Pcl5p on the high copy plasmid should lead to a stabilized Pcl5p.

However, this study has shown that Pcl5p constitutively interacts with Pho85p but also with Pho81p whose requirement for the Gen $4 p$ stability regulation is quite puzzling. The interaction of Pcl5p and Pho81p is not required for Gcn4p degradation because a similar degradation of Gcn $4 p$ has been reported in the presence and absence of functional Pho81p in sated cells (Bömeke et al., 2006). Furthermore, Pho81p is not required for the interaction of Pcl5p and Pho85p under non-starvation and starvation conditions (Figure 18B) whereas it is required for the stabilization of Gcn4p in response to amino acid limitation (Bömeke et al., 2006). This suggests an inhibitory function of Pho81p on Pho85p/Pcl5p activity in amino acid starved cells when Gen $4 p$ is necessary to overcome starvation. This would be analogous to the situation described for Pho81p function on Pho85p/Pho80p activity in phosphate metabolism. Pho81p constitutively interacts with Pho85p/Pho80p complexes but only develops its inhibitory function when phosphate becomes limiting. A small ligand, inositol heptakisphosphate $\left(\mathrm{IP}_{7}\right)$, has been recently shown to inactivate Pho85p/Pho80p kinase activity by a noncovalent binding that induces additional interactions between Pho81p and Pho85p/Pho80p which prevent that the substrate accesses the active site of the kinase (Lee et al., 2008). The CKI p27 (Kip1) has also been shown to interact constitutively with $\mathrm{Cdk} 4 /$ cyclin D but regulates kinase activity dependent on its phosphorylation status (James et 
al., 2008). p27/Kip1 phosphorylation on two tyrosine residues by protein kinase B promotes assembly of p27/CDK4/cyclin D complexes but requires additional tyrosine phosphorylation of p27 for the catalytic activation of the kinase complexes (Larrea et al., 2008). It therefore depends on the phosphorylation state whether p27 acts as assembly factor or inhibitor for CDK4/cyclin D (James et al., 2008) and it was suggested that this enables the cells to rapidly activate kinase activity when needed while maintaining a pool of preformed complexes (Ray et al., 2009). It is possible that Pho81p constitutively interacts with Pho85p/Pcl5p to inhibit the kinase activity under starvation conditions but this is contradictory to the constitutive Gcn4p degradation in cells lacking Pcl7p (Bömeke et al., 2006) or the decreased stabilization of Gen $4 p$ in starved cells expressing a stabilized Pcl5p-variant (Aviram et al., 2008). Furthermore, neither deletion nor overexpression of PHO81 changed the sensitivity to 3AT even when high amounts of this competitive inhibitor were used (Figure 23 and data not shown, respectively) conditions that were shown to stabilize Gen4p. A highly speculative possibility is that Pho81p does not inhibit the Pho85p/Pc15p activity but reduces the efficiency of Pcl5p association with Pho85p, thereby promoting the interaction of Pho85p with another cyclin when the Pcl5p mediated kinase activity is not required. This would explain why Gen $4 p$ is constitutively degraded in pho81 deficient cells. Another explanation of the constitutive Gcn $4 p$ degradation in pho81 impaired cells is that Pho81p functions as assembly factor for other cyclins, for example Pho80p or Pcl7p, and that the lack of Pho81p diminishes their interaction thereby promoting Pho85p/Pcl5p complex formation In case of Pcl7p, overexpression of $\mathrm{PHO} 81$ did not reduce the increased hypersensitivity to 3AT when PCL7 is overexpressed (data not shown). In contrast, pho81 mutant cells that overexpress PCL7 seem to be slightly impaired in growth on YNB medium with 3AT compared to PHO81 cells overexpressing PCL7 (Figure 23). This suggests that Pho81p has an inhibitory effect on PCL7 overexpression although overexpression of PHO81 alone had no obvious effect. However, this effect was not always observed and if so, seems to be very weak. Furthermore, it has to be tested if this effect can be suppressed by PHO81 expression in pho81 mutant cells.

Moreover, it is possible that Pho81p affects the Gen4p stability control indirectly by the regulation of another Pho85p/cyclin complex. Since Pho85p/Pho80p kinases are regulated by Pho81 $p$ and involved in the control of many different factors, e. g. Rim15p, Pho4p, which are involved in many cellular processes and different pathways it is likely that the loss of Pho81p due to a PHO81 deletion leads to an indirect impact on the Gcn4p stability control. Nevertheless, the role of Pho81p in Gcn $4 p$ stability regulation and its interaction with Pcl5p is still puzzling and requires future investigations. 


\subsubsection{Competitive binding of Pcl7p to Pho85p counteracts Pho85p/Pcl5p complex formation}

The cyclin Pcl7p in association with Pho85p was initially shown to be involved in the glycogen metabolism. Furthermore, the activity of this complex is regulated in a phosphate dependent manner by the CKI Pho81p (Lee et al., 2000). Later, additional roles of the cyclin were observed including the requirement of Pcl7p for stabilization of the transcription factor Gcn4p (Bömeke et al., 2006). Deletion of PCL7 results in rapid degradation of Gen4p in amino starved cells, conditions that normally stabilize the protein. Moreover, Pcl7p is a nuclear and stable protein independent of the amino acid availability and associates with Pho85p as well as with the CKI Pho81p in sated and amino acid starved cells (Bömeke et al., 2006). It was assumed that the function of Pcl7p in the Gcn $4 p$ stability control is an antagonistic role to Pcl5p by competitive binding to the CDK Pho85p. In addition, it was supposed that amino acid starvation results in a shift of the equilibrium towards more Pho85p/Pcl7p complexes and less Pho85p/Pcl5p complexes due to dissociation of the latter complexes. This was supported by the fact that overexpression of PCL7 leads to a strong stabilization of Gen $4 p$ under amino acid starvation conditions (Bömeke et al., 2006). Nevertheless, this was contradictory since Pcl7p was not required for the disruption of the Pho85p/Pcl5p and Pho81p/Pcl5p interaction. With regard to the finding described here, that Pcl5p interacts with both Pho85p and Pho81p independent of the amino acid availability, it is likely that the function of Pcl7p in the Gcn $4 p$ stability regulation is its antagonistic binding to Pho85p. One prediction of this model (Figure 26) is that high amounts of Pcl7p that compete with Pcl5p for binding to Pho85p should result in a shift to less Pho85p/Pcl5p complexes and therefore a less efficient Gcn4p degradation (Figure 26A). Furthermore, an increase of Pho85p molecules in the cell should lead to a bigger pool of unbound Pho85p in the cell thereby reducing the competition of the cyclins to bind free Pho85p. Hence, overexpression of PHO85 should alleviate the complex formation with Pc15p and lead to a rapid Gcn $4 p$ decay in starved cells similarly to the fast Gcn $4 p$ turnover in pcl7 mutant cells under amino acid starvation conditions as described by Bömeke et al. (2006) (Figure 26D and C, respectively). As demonstrated in this work, both predictions turned out to be right. High amounts of Pcl7p lead to a slightly reduced Gcn $4 p$ degradation in sated cells (Figure 21) whereas overexpression of PHO85 has the opposite effect and leads to an impaired stabilization of Gcn $4 p$ under amino acid starvation conditions (Figure 20). Additionally, constitutive expression of PCL5 (Figure 26B) similarly affects the Gcn4p stabilization and results in rapid 
degradation of the transcription factor under amino acid depletion conditions (Figure 19). Although the negative effect of high levels of Pcl7p on Gcn $4 p$ degradation is hardly detectable in sated cells, the antagonistic function of Pcl7p is additionally corroborated by the fact that overexpression of PCL7 slightly induces GCN4 overexpression toxicity (Figure 22). A competitive action of Pcl7p and Pcl5p in binding to Pho85p is also supported by the fact that concomitant overexpression of either PCL5 or PHO85 suppresses the toxic effect of high levels of Gcn $4 p$ when PCL7 is overexpressed in PCL5 PHO85 wild type cells (Figure 22).

A
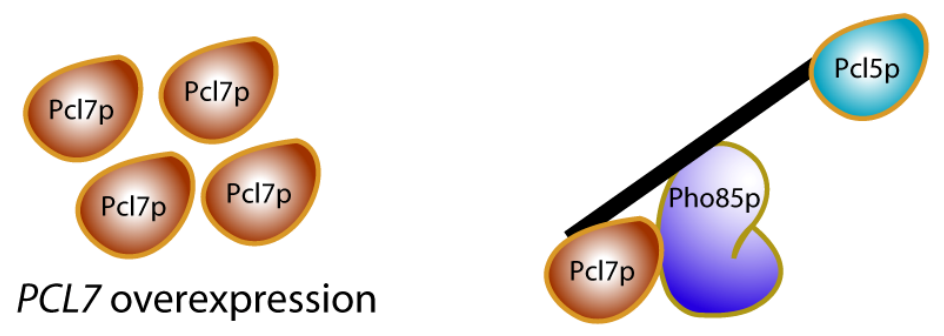

Stabilization

B

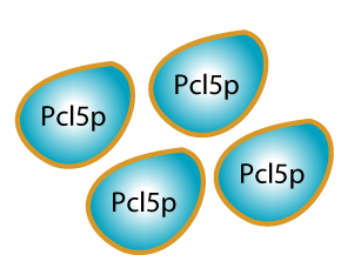

PCL5 overexpression

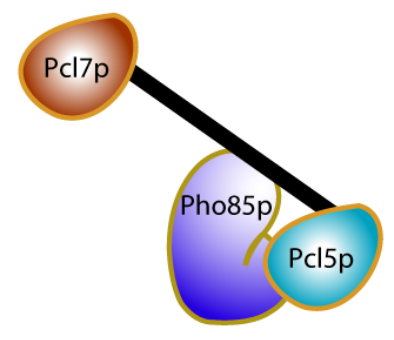

Degradation

C

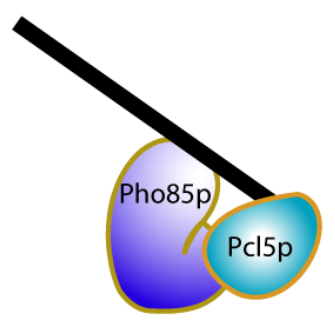

Degradation

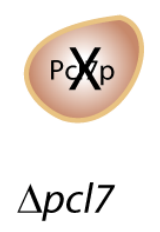

D

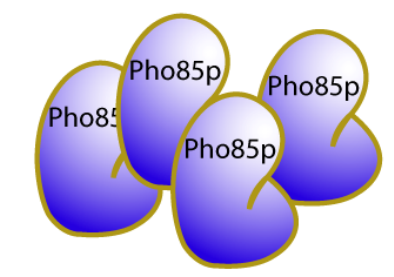

PHO85 overexpression

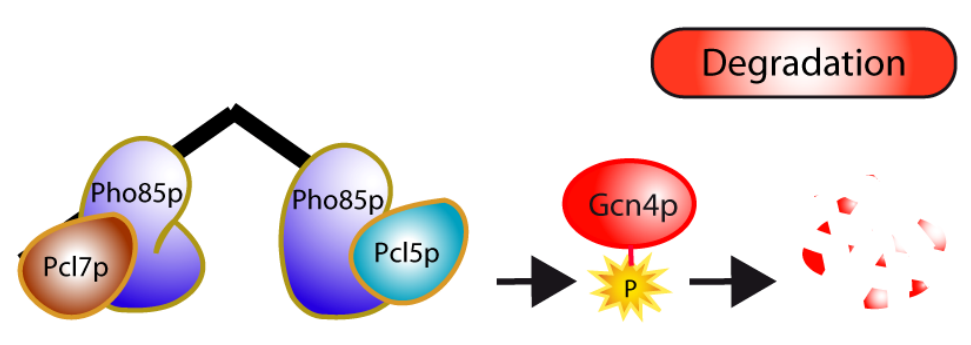

Figure 26: Model of competitive binding of Pcl7p and Pcl5p to Pho85p. (A) Overexpression of PCL7 leads to a shift towards more Pho85p/Pcl7p and less Pho85p/Pc15p complexes resulting in less efficient Gcn $4 p$ degradation and a stabilization of Gcn4p. (B) PCL5 overexpression results in a shift towards more Pho85p/Pc15p complexes and constitutive Gcn $4 p$ degradation. (C) The lack of Pcl7p and therefore the lack of competition to bind to Pho85p promotes Pho85p/Pcl5p complex formation and leads to constitutive degradation of Gcn4p. (D) Overexpression of PHO85 leads to a bigger pool of free Pho85p which enables Pcl7p and Pcl5p to bind without competition and results in constitutive Gen4 decay. 
In contrast to previous studies, hypersensitivity of pcl7 mutant cells to $100 \mathrm{mM} 3 \mathrm{AT}$ was not observed (Figure 23) whereas overexpression of PCL7 resulted in reduced growth in the presence of $100 \mathrm{mM}$ 3AT (Figure 23) as previously reported (Bömeke et al., 2006). Interestingly, a pcl5 defective strain showed a similar growth reduction in the presence of 100 $\mathrm{mM} 3 \mathrm{AT}$. But neither PCL7 overexpression nor the lack of Pcl5p led to higher sensitivity to a concentration of $10 \mathrm{mM} 3 \mathrm{AT}$. In contrast, a slightly increase in PCL7 overexpression-induced sensitivity to 3AT might be possible in pho81 mutant cells compared to PHO81 wild-type cells (Figure 23) as mentioned above. Although this is not sufficiently investigated as it suggests that the Pho81p inhibitory activity is directed towards Pcl7p in an unknown manner and not directly to the Pho85p/Pcl5p kinase activity since the lack of PHO81 alone did not affect the sensitivity to 3AT (Figure 23).

A possible explanation for the 3AT-sensitive phenotype is a toxic effect of Gen $4 p$ due to its strong stabilization. This may lead to a similar toxic effect as described for Gen $4 p$ overexpression (Tavernarakis and Thireos, 1995). However, simultaneous overexpression of PHO85 again counteracted the PCL7 overexpression phenotype (Figure 23) which is consistent with the assumption that Pc15p can not bind to Pho85p because high amounts of the stable cyclin Pcl7p displace Pcl5p.

\subsubsection{Model of the Gcn4p stability regulation in the yeast $S$. cerevisiae}

The rapid degradation of the transcription factor Gen4p is dependent on the CDK Pho85p in association with its activating cyclin Pcl5p. Within this work, it has been demonstrated that the cyclin Pc15p and the CDK Pho85p interact under non-starvation and starvation conditions. Similarly, a constitutive interaction between the CKI Pho81p and Pcl5p was shown. Furthermore, the cyclin Pcl7p, which is also able to bind Pho85p and Pho81p, was shown to counteract the Pho85p/Pc15p activity by competition of binding to the CDK Pho85p (Figure 26). An increase of Pc15p or Pho85p molecules as well as the lack of Pcl7p leads to a shift towards more Pho85p/Pc15p complexes that initiate the Gcn $4 p$ degradation whereas overexpression of PCL7 has an opposite effect and decreases the efficiency of Pho85p/Pcl5p complex formation. It has been previously demonstrated and also observed in this study, that amino acid starvation leads to a drastic reduction of Pcl5p in the cell (Shemer et al., 2002). This is presumably due to the reduced overall translation under amino acid depletion conditions combined with the high constitutive turnover of the cyclin as recently described (Aviram et al., 2008). Since PCL5 has been shown to be transcriptionally induced by Gen4p 
(Jia et al., 2000), it is very likely that other mechanisms are involved that control the translation efficiency. It has been shown that upstream sequence elements of many genes have a post-transcriptionally regulatory function on gene expression in response to stress conditions (Lawless et al., 2009). Translational control of gene expression in fungi is often mediated by small upstream open reading frames (uORF) which can affect the translation by different mechanisms that can lead to up and downregulation (Hood et al., 2009). The best characterized example is the mRNA of GCN4 that contains four uORFs that lead to an increased translation of the GCN4 ORF under conditions of amino acid limitation and therefore to an increased amount of the transcriptional activator Gcn $4 p$ to induce a response that enables the cells to overcome the starvation (Hinnebusch, 2005). Since PCL5 expression is reduced in amino acid starved yeast cells, a mechanism similar to the translational control of GCN4 expression is unlikely but it shows that uORF can function in a context-dependent manner rather than just inhibiting. In contrast to the GCN4 translational control, the expression of the G1 cyclin encoding gene CLN3 is repressed by one uORF in its mRNA 5 ' leader on translational level. Removal of the uORF resulted in an increased expression of CLN3 (Polymenis and Schmidt, 1997). The 5' untranslated region of the PCL5 mRNA is extended, indicative for translational control of expression, and contains two uORFs which were shown to be conserved in six other Saccharomyces species (Zhang and Dietrich, 2005; Cvijovic et al., 2007). It is therefore quite conceivable that despite the transcriptional induction, the level of Pc15p in starved cells is very low due to a translational regulatory mechanism that represses Pcl5p synthesis but which is at this point unresolved and remains as a future task.

However, amino acid starvation drastically reduces the amount of Pcl5p in the cell. Less Pcl5p molecules in turn lead to a reduced chance to bind Pho85p and therefore to less Pho85p/Pcl5p complexes in the cell which are able to initiate the Gcn $4 p$ degradation and hence, in an increased stability of the transcription factor in amino acid starved cells. This is supported by the impaired Gcn4p stabilization when the amount of Pcl5p is increased in amino acid starved cells either by constitutive overexpression as demonstrated in this work or by stabilization of the cyclin (Aviram et al., 2008). It furthermore explains that Gcn4p is not stabilized under amino acid starvation conditions in pcl7 defective cells. The lack of Pcl7p presumably enables the very few molecules of Pcl5p that are synthesized to bind and to activate Pho85p in starved cells which is normally counteracted by the stable cyclin Pcl7p. This also explains that overexpression results in a strong stabilization of Gcn $4 p$ under starvation conditions in cells overexpressing PCL7. An increased number of Pcl7p molecules 
versus a decreased number of Pcl5p molecules lead to a shift of the normally balanced equilibrium in sated cells towards much more Pho85p/Pcl7p complexes and very few or none Pho85p/Pcl5p complexes. It has been proposed by Aviram and co-workers that the number of Pcl5p molecules per cell under nutrient rich conditions is around ten. Although the data was not shown, this explains that a nine to thirteen fold myc tag is required to obtain reliable detection of the protein under non-starvation conditions whereas Pc15p is hardly detectable in starved cells when expressed from the native promoter. Nevertheless, this small amount is enough to initiate the rapid degradation of Gen $4 p$ in a nutrient rich environment and less Pcl5p under conditions of amino acid limitation is still sufficient as long as free Pho85p molecules are available for complex formation with Pcl5p. Although it can not be excluded that other processes, for example inhibition of the kinase activity, participate in the control of Gcn $4 p$ stability, the main regulatory mechanism is likely to be the reduced amount of Pcl5p that competes for binding to Pho85p to initiate the Gcn $4 p$ degradation under amino acid starvation conditions. Therefore, the way of contribution of Pcl7p within the stability regulation of Gcn $4 p$ seems to be a more indirect competitive rather than direct mechanism but this has to be verified. When the observed effects on Gen $4 p$ degradation caused by the presence or absence of Pcl7p, i. e. either stabilization of Gcn4p by an increased amount of Pcl7p molecules or constitutive turnover of the transcription factor in cells lacking PCL7 independent of the amino acid availability, are just due to different concentrations of the cyclin, other stable Pho85p cyclins like Pho80p should have similar effects. This means that deletion of $\mathrm{PHO} 80$ should result in constitutive expression whereas overexpression should lead to a stabilization of Gcn4p. However, this has not been tested so far and remains for further studies. Furthermore, the influence of the extended uORF containing 5 ' untranslated region of the PCL5 mRNA has not been investigated. It is conceivable that the uORFs in the 5' UTR of the PCL5 mRNA have a negative effect on translation, which might be more pronounced under amino acid limitation conditions since the amount of Pcl5p decreases in amino acid cells although a transcriptional induction of PCL5 under this condition has been shown that depends on Gen4p (Jia et al., 2000). 

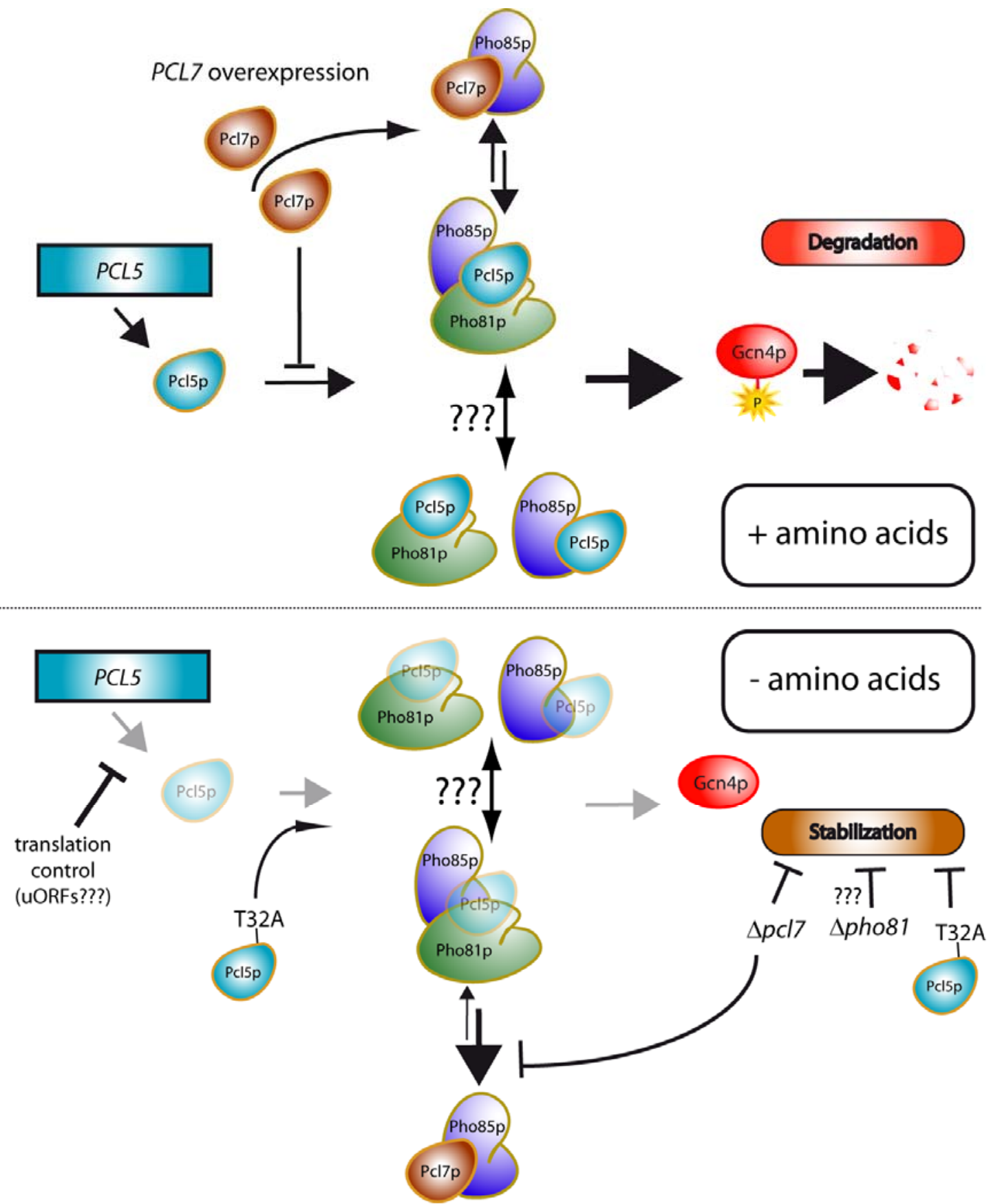

Figure 27: Model of the Gen4p stability regulation in S. cerevisiae. (Top) In sated cells, sufficient Pcl5p molecules are available that compete with Pcl7p (and presumably other Pho85p interacting cyclins) for Pho85p and Pho81p binding to form active Pho85p/Pcl5p and Pho81p/Pcl5p or possibly ternary complexes. The active Pho85p/Pcl5p complexes phosphorylate Gcn $4 p$ which leads to its rapid degradation via the ubiquitin-proteasome pathway. Overexpression of PCL7 counteracts complex formation of Pc15p with Pho85p (and Pho81p) due to the competitive Pcl7p association which leads to Gcn $4 p$ stabilization. (Bottom) Under amino acid starvation conditions, the equilibrium between Pcl5p and Pcl7p (and other cyclins) is shifted towards less Pcl5p molecules due to its rapid degradation combined with its decreased translation observed under this condition. This finally results in much less Pcl5p-containing complexes that are able to initiate the Gcn $4 p$ degradation which leads to the stabilization of Gcn $4 p$. Mutation of the Pc15p degradation signal (T32A) decreases the turnover of the cyclin resulting in more Pcl5p cyclins that compete for kinase binding which in turn counteracts Gcn4p stabilization. Deletion of PCL7 (or presumably other cyclins) reduces the competition for Pcl5p to form an active kinase/cyclin complex causing an impaired Gcn4p stabilization. Deletion of PHO81 counteracts Gen4p stabilization by an unknown mechanism. 
In conclusion, the stability regulation of the key regulator of the general amino acid control network in S. cerevisiae mainly depends on the amount of the cyclin Pcl5p that competes with a pool of other cyclin molecules for the formation of a Pho85p/cyclin complex. In sated cells, sufficient amounts of Pcl5p are present to initiate in complex with Pho85p the Gcn $4 p$ degradation. In contrast, amino acid starvation conditions lead to a significant decreased amount of Pcl5p molecules due to its rapid and constitutive turnover combined with a putative translational control mechanism and therefore much less Pho85p/Pcl5p complexes are formed that are able to phosphorylate Gcn $4 p$ to be finally degraded via the ubiquitinproteasome pathway, thus Gcn4p becomes stabilized. 


\section{References}

Albrecht, G., Mösch, H.U., Hoffmann, B., Reusser, U., and Braus, G.H. (1998). Monitoring the Gcn 4 protein-mediated response in the yeast Saccharomyces cerevisiae. J Biol Chem 273, 12696-12702.

Aviram, S., Simon, E., Gildor, T., Glaser, F., and Kornitzer, D. (2008). Autophosphorylationinduced degradation of the Pho85 cyclin Pcl5 is essential for response to amino acid limitation. Mol Cell Biol 28, 6858-6869.

Barrett, C.P., and Noble, M.E. (2005). Molecular motions of human cyclin-dependent kinase 2. J Biol Chem 280, 13993-14005.

Bloom, J., and Cross, F.R. (2007). Multiple levels of cyclin specificity in cell-cycle control. Nat Rev Mol Cell Biol 8, 149-160.

Bömeke, K. (2006). Stability regulation of Gcn4p in Saccharomyces cerevisiae. Dissertation. Georg-August Universität. Göttingen.

Bömeke, K., Pries, R., Korte, V., Scholz, E., Herzog, B., Schulze, F., and Braus, G.H. (2006). Yeast Gcn $4 p$ stabilization is initiated by the dissociation of the nuclear Pho85p/Pcl5p complex. Mol Biol Cell 17, 2952-2962.

Bosu, D.R., and Kipreos, E.T. (2008). Cullin-RING ubiquitin ligases: global regulation and activation cycles. Cell Div 3, 7.

Brachmann, C.B., Davies, A., Cost, G.J., Caputo, E., Li, J., Hieter, P., and Boeke, J.D. (1998). Designer deletion strains derived from Saccharomyces cerevisiae S288C: a useful set of strains and plasmids for PCR-mediated gene disruption and other applications. Yeast 14, 115-132.

Bradford, M.M. (1976). A rapid and sensitive method for the quantitation of microgram quantities of protein utilizing the principle of protein-dye binding. Anal Biochem 72, 248-254.

Braus, G.H., Grundmann, O., Brückner, S., and Mösch, H.U. (2003). Amino acid starvation and Gen4p regulate adhesive growth and FLO11 gene expression in Saccharomyces cerevisiae. Mol Biol Cell 14, 4272-4284.

Brown, N.R., Noble, M.E., Endicott, J.A., Garman, E.F., Wakatsuki, S., Mitchell, E., Rasmussen, B., Hunt, T., and Johnson, L.N. (1995). The crystal structure of cyclin A. Structure 3, 1235-1247.

Brown, N.R., Noble, M.E., Endicott, J.A., and Johnson, L.N. (1999). The structural basis for specificity of substrate and recruitment peptides for cyclin-dependent kinases. Nat Cell Biol 1, 438-443.

Carsiotis, M., Jones, R.F., and Wesseling, A.C. (1974). Cross-pathway regulation: histidinemediated control of histidine, tryptophan, and arginine biosynthetic enzymes in Neurospora crassa. J Bacteriol 119, 893-898.

Cherkasova, V., Qiu, H., and Hinnebusch, A.G. (2010). Snf1 promotes phosphorylation of the alpha subunit of eukaryotic translation initiation factor 2 by activating Gen2 and inhibiting phosphatases Glc7 and Sit4. Mol Cell Biol 30, 2862-2873.

Cherkasova, V.A., and Hinnebusch, A.G. (2003). Translational control by TOR and TAP42 through dephosphorylation of eIF2alpha kinase GCN2. Genes Dev 17, 859-872.

Chi, Y., Huddleston, M.J., Zhang, X., Young, R.A., Annan, R.S., Carr, S.A., and Deshaies, R.J. (2001). Negative regulation of Gcn4 and Msn2 transcription factors by Srb10 cyclin-dependent kinase. Genes Dev 15, 1078-1092.

Chrousos, G.P., and Kino, T. (2009). Glucocorticoid signaling in the cell. Expanding clinical implications to complex human behavioral and somatic disorders. Ann N Y Acad Sci 1179, 153-166. 
Corneo, B., Benmerah, A., and Villartay, J.P. (2002). A short peptide at the C terminus is responsible for the nuclear localization of RAG2. Eur J Immunol 32, 2068-2073.

Correia, I., Alonso-Monge, R., and Pla, J. (2010). MAPK cell-cycle regulation in Saccharomyces cerevisiae and Candida albicans. Future Microbiol 5, 1125-1141.

Costa-Mattioli, M., Gobert, D., Harding, H., Herdy, B., Azzi, M., Bruno, M., Bidinosti, M., Ben Mamou, C., Marcinkiewicz, E., Yoshida, M., Imataka, H., Cuello, A.C., Seidah, N., Sossin, W., Lacaille, J.C., Ron, D., Nader, K., and Sonenberg, N. (2005). Translational control of hippocampal synaptic plasticity and memory by the eIF2alpha kinase GCN2. Nature 436, 1166-1173.

Cvijovic, M., Dalevi, D., Bilsland, E., Kemp, G.J., and Sunnerhagen, P. (2007). Identification of putative regulatory upstream ORFs in the yeast genome using heuristics and evolutionary conservation. BMC Bioinformatics 8, 295.

de Gouvea, P.F., Soriani, F.M., Malavazi, I., Savoldi, M., Goldman, M.H., Loss, O., Bignell, E., da Silva Ferreira, M.E., and Goldman, G.H. (2008). Functional characterization of the Aspergillus fumigatus PHO80 homologue. Fungal Genet Biol 45, 1135-1146.

Deshaies, R.J., and Joazeiro, C.A. (2009). RING domain E3 ubiquitin ligases. Annu Rev Biochem 78, 399-434.

Doonan, J.H., and Kitsios, G. (2009). Functional evolution of cyclin-dependent kinases. $\mathrm{Mol}$ Biotechnol 42, 14-29.

Draviam, V.M., Orrechia, S., Lowe, M., Pardi, R., and Pines, J. (2001). The localization of human cyclins $\mathrm{B} 1$ and $\mathrm{B} 2$ determines $\mathrm{CDK} 1$ substrate specificity and neither enzyme requires MEK to disassemble the Golgi apparatus. J Cell Biol 152, 945-958.

Drysdale, C.M., Duenas, E., Jackson, B.M., Reusser, U., Braus, G.H., and Hinnebusch, A.G. (1995). The transcriptional activator GCN4 contains multiple activation domains that are critically dependent on hydrophobic amino acids. Mol Cell Biol 15, 1220-1233.

Echalier, A., Endicott, J.A., and Noble, M.E. (2009). Recent developments in cyclindependent kinase biochemical and structural studies. Biochim Biophys Acta 1804, 511-519.

Engelberg, D., Klein, C., Martinetto, H., Struhl, K., and Karin, M. (1994). The UV response involving the Ras signaling pathway and AP-1 transcription factors is conserved between yeast and mammals. Cell 77, 381-390.

Finley, D. (2009). Recognition and processing of ubiquitin-protein conjugates by the proteasome. Annu Rev Biochem 78, 477-513.

Fiserova, J., and Goldberg, M.W. (2010). Nucleocytoplasmic transport in yeast: a few roles for many actors. Biochem Soc Trans 38, 273-277.

Franceschi, R.T., Ge, C., Xiao, G., Roca, H., and Jiang, D. (2009). Transcriptional regulation of osteoblasts. Cells Tissues Organs 189, 144-152.

Friesen, H., Murphy, K., Breitkreutz, A., Tyers, M., and Andrews, B. (2003). Regulation of the yeast amphiphysin homologue Rvs167p by phosphorylation. Mol Biol Cell 14, 3027-3040.

Galagan, J.E., Calvo, S.E., Cuomo, C., Ma, L.J., Wortman, J.R., Batzoglou, S., Lee, S.I., Basturkmen, M., Spevak, C.C., Clutterbuck, J., Kapitonov, V., Jurka, J., Scazzocchio, C., Farman, M., Butler, J., Purcell, S., Harris, S., Braus, G.H., Draht, O., Busch, S., D'Enfert, C., Bouchier, C., Goldman, G.H., Bell-Pedersen, D., Griffiths-Jones, S., Doonan, J.H., Yu, J., Vienken, K., Pain, A., Freitag, M., Selker, E.U., Archer, D.B., Penalva, M.A., Oakley, B.R., Momany, M., Tanaka, T., Kumagai, T., Asai, K., Machida, M., Nierman, W.C., Denning, D.W., Caddick, M., Hynes, M., Paoletti, M., Fischer, R., Miller, B., Dyer, P., Sachs, M.S., Osmani, S.A., and Birren, B.W. (2005). Sequencing of Aspergillus nidulans and comparative analysis with A. fumigatus and A. oryzae. Nature 438, 1105-1115. 
Goossens, A., Dever, T.E., Pascual-Ahuir, A., and Serrano, R. (2001). The protein kinase Gcn2p mediates sodium toxicity in yeast. J Biol Chem 276, 30753-30760.

Granovskaia, M.V., Jensen, L.J., Ritchie, M.E., Toedling, J., Ning, Y., Bork, P., Huber, W., and Steinmetz, L.M. (2010). High-resolution transcription atlas of the mitotic cell cycle in budding yeast. Genome Biol 11, R24.

Guthrie, C., and Fink, G.R. (1991). Guide to Yeast Genetics and Molecular Biology. Methods Enzymol. 194.

Hahn, S., Maurer, P., Caesar, S., and Schlenstedt, G. (2008). Classical NLS proteins from Saccharomyces cerevisiae. J Mol Biol 379, 678-694.

Hershko, A. (2005). The ubiquitin system for protein degradation and some of its roles in the control of the cell-division cycle (Nobel lecture). Angew Chem Int Ed Engl 44, 59325943.

Hinnebusch, A.G. (2005). Translational regulation of GCN4 and the general amino acid control of yeast. Annu Rev Microbiol 59, 407-450.

Hirsch, C., Gauss, R., Horn, S.C., Neuber, O., and Sommer, T. (2009). The ubiquitylation machinery of the endoplasmic reticulum. Nature 458, 453-460.

Holmes, D.S., and Quigley, M. (1981). A rapid boiling method for the preparation of bacterial plasmids. Anal Biochem 114, 193-197.

Hood, H.M., Neafsey, D.E., Galagan, J., and Sachs, M.S. (2009). Evolutionary roles of upstream open reading frames in mediating gene regulation in fungi. Annu Rev Microbiol 63, 385-409.

Hope, I.A., and Struhl, K. (1986). Functional dissection of a eukaryotic transcriptional activator protein, GCN4 of yeast. Cell 46, 885-894.

Hu, F., Gan, Y., and Aparicio, O.M. (2008). Identification of Clb2 residues required for Swe1 regulation of Clb2-Cdc28 in Saccharomyces cerevisiae. Genetics 179, 863-874.

Huang, D., Friesen, H., and Andrews, B. (2007a). Pho85, a multifunctional cyclin-dependent protein kinase in budding yeast. Mol Microbiol 66, 303-314.

Huang, D., Kaluarachchi, S., van Dyk, D., Friesen, H., Sopko, R., Ye, W., Bastajian, N., Moffat, J., Sassi, H., Costanzo, M., and Andrews, B.J. (2009). Dual regulation by pairs of cyclin-dependent protein kinases and histone deacetylases controls G1 transcription in budding yeast. PLoS Biol 7, e1000188.

Huang, D., Moffat, J., Wilson, W.A., Moore, L., Cheng, C., Roach, P.J., and Andrews, B. (1998). Cyclin partners determine Pho85 protein kinase substrate specificity in vitro and in vivo: control of glycogen biosynthesis by Pcl8 and Pcl10. Mol Cell Biol 18, 3289-3299.

Huang, K., Ferrin-O'Connell, I., Zhang, W., Leonard, G.A., O'Shea, E.K., and Quiocho, F.A. (2007b). Structure of the Pho85-Pho80 CDK-cyclin complex of the phosphateresponsive signal transduction pathway. Mol Cell 28, 614-623.

Inoue, H., Nojima, H., and Okayama, H. (1990). High efficiency transformation of Escherichia coli with plasmids. Gene 96, 23-28.

Irniger, S., Piatti, S., Michaelis, C., and Nasmyth, K. (1995). Genes involved in sister chromatid separation are needed for B-type cyclin proteolysis in budding yeast. Cell 81, 269-278.

Ito, H., Fukuda, Y., Murata, K., and Kimura, A. (1983). Transformation of intact yeast cells treated with alkali cations. J Bacteriol 153, 163-168.

Jackson, L.P., Reed, S.I., and Haase, S.B. (2006). Distinct mechanisms control the stability of the related S-phase cyclins Clb5 and Clb6. Mol Cell Biol 26, 2456-2466.

Jäkel, S., and Görlich, D. (1998). Importin beta, transportin, RanBP5 and RanBP7 mediate nuclear import of ribosomal proteins in mammalian cells. EMBO J 17, 4491-4502.

James, M.K., Ray, A., Leznova, D., and Blain, S.W. (2008). Differential modification of p27Kip1 controls its cyclin D-cdk4 inhibitory activity. Mol Cell Biol 28, 498-510. 
Jia, M.H., Larossa, R.A., Lee, J.M., Rafalski, A., Derose, E., Gonye, G., and Xue, Z. (2000). Global expression profiling of yeast treated with an inhibitor of amino acid biosynthesis, sulfometuron methyl. Physiol Genomics 3, 83-92.

Jonkers, W., and Rep, M. (2009). Lessons from fungal F-box proteins. Eukaryot Cell 8, 677695.

Kaffman, A., and O'Shea, E.K. (1999). Regulation of nuclear localization: a key to a door. Annu Rev Cell Dev Biol 15, 291-339.

Kalderon, D., Richardson, W.D., Markham, A.F., and Smith, A.E. (1984). Sequence requirements for nuclear location of simian virus 40 large-T antigen. Nature 311, 3338.

Keaton, M.A., Bardes, E.S., Marquitz, A.R., Freel, C.D., Zyla, T.R., Rudolph, J., and Lew, D.J. (2007). Differential susceptibility of yeast S and M phase CDK complexes to inhibitory tyrosine phosphorylation. Curr Biol 17, 1181-1189.

Kilberg, M.S., Shan, J., and Su, N. (2009). ATF4-dependent transcription mediates signaling of amino acid limitation. Trends Endocrinol Metab 20, 436-443.

Kim, S.H., Kim, H.J., Kim, S., and Yim, J. (2010). Drosophila Cand1 regulates Cullin3dependent E3 ligases by affecting the neddylation of Cullin3 and by controlling the stability of Cullin3 and adaptor protein. Dev Biol.

Kleinschmidt, M., Grundmann, O., Blüthgen, N., Mösch, H.U., and Braus, G.H. (2005). Transcriptional profiling of Saccharomyces cerevisiae cells under adhesion-inducing conditions. Mol Genet Genomics 273, 382-393.

Klopotowski, T., and Wiater, A. (1965). Synergism of aminotriazole and phosphate on the inhibition of yeast imidazole glycerol phosphate dehydratase. Arch Biochem Biophys 112, 562-566.

Knight, J.P., Daly, T.M., and Bergman, L.W. (2004). Regulation by phosphorylation of Pho81p, a cyclin-dependent kinase inhibitor in Saccharomyces cerevisiae. Curr Genet 46, 10-19.

Koepp, D.M., Wong, D.H., Corbett, A.H., and Silver, P.A. (1996). Dynamic localization of the nuclear import receptor and its interactions with transport factors. J Cell Biol 133, 1163-1176.

Komeili, A., and O'Shea, E.K. (2000). Nuclear transport and transcription. Curr Opin Cell Biol 12, 355-360.

Kornitzer, D., Raboy, B., Kulka, R.G., and Fink, G.R. (1994). Regulated degradation of the transcription factor Gcn4. EMBO J 13, 6021-6030.

Kosugi, S., Hasebe, M., Matsumura, N., Takashima, H., Miyamoto-Sato, E., Tomita, M., and Yanagawa, H. (2009). Six classes of nuclear localization signals specific to different binding grooves of importin alpha. J Biol Chem 284, 478-485.

Kubota, H., Ota, K., Sakaki, Y., and Ito, T. (2001). Budding yeast GCN1 binds the GI domain to activate the eIF2alpha kinase GCN2. J Biol Chem 276, 17591-17596.

Laemmli, U.K. (1970). Cleavage of structural proteins during the assembly of the head of bacteriophage T4. Nature 227, 680-685.

Lalioti, V., Pulido, D., and Sandoval, I.V. (2010). Cdk5, the multifunctional surveyor. Cell Cycle 9, 284-311.

Landschulz, W.H., Johnson, P.F., and McKnight, S.L. (1988). The leucine zipper: a hypothetical structure common to a new class of DNA binding proteins. Science $\mathbf{2 4 0}$, 1759-1764.

Larrea, M.D., Liang, J., Da Silva, T., Hong, F., Shao, S.H., Han, K., Dumont, D., and Slingerland, J.M. (2008). Phosphorylation of p27Kip1 regulates assembly and activation of cyclin D1-Cdk4. Mol Cell Biol 28, 6462-6472.

Larson, J.R., Kozubowski, L., and Tatchell, K. (2010). Changes in Bni4 localization induced by cell stress in Saccharomyces cerevisiae. J Cell Sci 123, 1050-1059. 
Lawless, C., Pearson, R.D., Selley, J.N., Smirnova, J.B., Grant, C.M., Ashe, M.P., Pavitt, G.D., and Hubbard, S.J. (2009). Upstream sequence elements direct posttranscriptional regulation of gene expression under stress conditions in yeast. BMC Genomics 10, 7.

Lee, M., O'Regan, S., Moreau, J.L., Johnson, A.L., Johnston, L.H., and Goding, C.R. (2000). Regulation of the Pcl7-Pho85 cyclin-cdk complex by Pho81. Mol Microbiol 38, 411422.

Lee, Y.S., Huang, K., Quiocho, F.A., and O'Shea, E.K. (2008). Molecular basis of cyclinCDK-CKI regulation by reversible binding of an inositol pyrophosphate. Nat Chem Biol 4, 25-32.

Lee, Y.S., Mulugu, S., York, J.D., and O'Shea, E.K. (2007). Regulation of a cyclin-CDKCDK inhibitor complex by inositol pyrophosphates. Science 316, 109-112.

Liu, J., and Kipreos, E.T. (2000). Evolution of cyclin-dependent kinases (CDKs) and CDKactivating kinases (CAKs): differential conservation of CAKs in yeast and metazoa. Mol Biol Evol 17, 1061-1074.

Lolli, G. (2010). Structural dissection of cyclin dependent kinases regulation and protein recognition properties. Cell Cycle 9.

Lopes, J.P., Oliveira, C.R., and Agostinho, P. (2010). Neurodegeneration in an Abeta-induced model of Alzheimer's disease: the role of Cdk5. Aging Cell 9, 64-77.

Makkerh, J.P., Dingwall, C., and Laskey, R.A. (1996). Comparative mutagenesis of nuclear localization signals reveals the importance of neutral and acidic amino acids. Curr Biol 6, 1025-1027.

Maniatis, T., Fritsch, E.F., and Sambrook, J. (1989). Molecular Cloning, a laboratory manual.Book 1. 2nd edn., Cold Spring Harbor Laboratory Press. Cold Spring Harbor, NY.

Mateo, F., Vidal-Laliena, M., Canela, N., Busino, L., Martinez-Balbas, M.A., Pagano, M., Agell, N., and Bachs, O. (2009). Degradation of cyclin A is regulated by acetylation. Oncogene 28, 2654-2666.

Mateo, F., Vidal-Laliena, M., Pujol, M.J., and Bachs, O. (2010). Acetylation of cyclin A: a new cell cycle regulatory mechanism. Biochem Soc Trans 38, 83-86.

Measday, V., McBride, H., Moffat, J., Stillman, D., and Andrews, B. (2000). Interactions between Pho85 cyclin-dependent kinase complexes and the Swi5 transcription factor in budding yeast. Mol Microbiol 35, 825-834.

Measday, V., Moore, L., Retnakaran, R., Lee, J., Donoviel, M., Neiman, A.M., and Andrews, B. (1997). A family of cyclin-like proteins that interact with the Pho85 cyclindependent kinase. Mol Cell Biol 17, 1212-1223.

Meimoun, A., Holtzman, T., Weissman, Z., McBride, H.J., Stillman, D.J., Fink, G.R., and Kornitzer, D. (2000). Degradation of the transcription factor Gen4 requires the kinase Pho85 and the SCF(CDC4) ubiquitin-ligase complex. Mol Biol Cell 11, 915927.

Mendenhall, M.D., and Hodge, A.E. (1998). Regulation of Cdc28 cyclin-dependent protein kinase activity during the cell cycle of the yeast Saccharomyces cerevisiae. Microbiol Mol Biol Rev 62, 1191-1243.

Merlet, J., Burger, J., Gomes, J.E., and Pintard, L. (2009). Regulation of cullin-RING E3 ubiquitin-ligases by neddylation and dimerization. Cell Mol Life Sci 66, 1924-1938.

Meussdoerffer, F., and Fink, G.R. (1983). Structure and expression of two aminoacyl-tRNA synthetase genes from Saccharomyces cerevisiae. J Biol Chem 258, 6293-6299.

Miller, M. (2009). The importance of being flexible: the case of basic region leucine zipper transcriptional regulators. Curr Protein Pept Sci 10, 244-269. 
Miller, M.E., and Cross, F.R. (2000). Distinct subcellular localization patterns contribute to functional specificity of the $\mathrm{Cln} 2$ and $\mathrm{Cln} 3$ cyclins of Saccharomyces cerevisiae. Mol Cell Biol 20, 542-555.

Moffat, J., and Andrews, B. (2004). Late-G1 cyclin-CDK activity is essential for control of cell morphogenesis in budding yeast. Nat Cell Biol 6, 59-66.

Mosammaparast, N., Jackson, K.R., Guo, Y., Brame, C.J., Shabanowitz, J., Hunt, D.F., and Pemberton, L.F. (2001). Nuclear import of histone H2A and H2B is mediated by a network of karyopherins. J Cell Biol 153, 251-262.

Mösch, H.U., Scheier, B., Lahti, R., Mantsala, P., and Braus, G.H. (1991). Transcriptional activation of yeast nucleotide biosynthetic gene ADE4 by GCN4. J Biol Chem 266, 20453-20456.

Mumberg, D., Muller, R., and Funk, M. (1994). Regulatable promoters of Saccharomyces cerevisiae: comparison of transcriptional activity and their use for heterologous expression. Nucleic Acids Res 22, 5767-5768.

Nash, P., Tang, X., Orlicky, S., Chen, Q., Gertler, F.B., Mendenhall, M.D., Sicheri, F., Pawson, T., and Tyers, M. (2001). Multisite phosphorylation of a CDK inhibitor sets a threshold for the onset of DNA replication. Nature 414, 514-521.

Natarajan, K., Meyer, M.R., Jackson, B.M., Slade, D., Roberts, C., Hinnebusch, A.G., and Marton, M.J. (2001). Transcriptional profiling shows that Gen4p is a master regulator of gene expression during amino acid starvation in yeast. Mol Cell Biol 21, 4347-4368.

Nishizawa, M., Kawasumi, M., Fujino, M., and Toh-e, A. (1998). Phosphorylation of Sic1, a cyclin-dependent kinase $(\mathrm{Cdk})$ inhibitor, by $\mathrm{Cdk}$ including Pho85 kinase is required for its prompt degradation. Mol Biol Cell 9, 2393-2405.

Nishizawa, M., Komai, T., Katou, Y., Shirahige, K., Ito, T., and Toh, E.A. (2008). Nutrientregulated antisense and intragenic RNAs modulate a signal transduction pathway in yeast. PLoS Biol 6, 2817-2830.

Nishizawa, M., Tanigawa, M., Hayashi, M., Maeda, T., Yazaki, Y., Saeki, Y., and Toh-e, A. (2010). Pho85 kinase, a cyclin-dependent kinase, regulates nuclear accumulation of the Rim101 transcription factor in the stress response of Saccharomyces cerevisiae. Eukaryot Cell 9, 943-951.

Ogawa, N., Noguchi, K., Sawai, H., Yamashita, Y., Yompakdee, C., and Oshima, Y. (1995). Functional domains of Pho81p, an inhibitor of Pho85p protein kinase, in the transduction pathway of Pi signals in Saccharomyces cerevisiae. Mol Cell Biol 15, 997-1004.

Pavletich, N.P. (1999). Mechanisms of cyclin-dependent kinase regulation: structures of Cdks, their cyclin activators, and Cip and INK4 inhibitors. J Mol Biol 287, 821-828.

Peters, J.M. (2006). The anaphase promoting complex/cyclosome: a machine designed to destroy. Nat Rev Mol Cell Biol 7, 644-656.

Pinson, B., Vaur, S., Sagot, I., Coulpier, F., Lemoine, S., and Daignan-Fornier, B. (2009). Metabolic intermediates selectively stimulate transcription factor interaction and modulate phosphate and purine pathways. Genes Dev 23, 1399-1407.

Piotrowska, M. (1980). Cross-Pathway regulation of ornithine carbamoyltransferase synthesis in Aspergillus nidulans. J Gen Microbiol 116, 335-339.

Polymenis, M., and Schmidt, E.V. (1997). Coupling of cell division to cell growth by translational control of the G1 cyclin CLN3 in yeast. Genes Dev 11, 2522-2531.

Pries, R., Bomeke, K., Draht, O., Kunzler, M., and Braus, G.H. (2004). Nuclear import of yeast Gcn4p requires karyopherins Srp1p and Kap95p. Mol Genet Genomics 271, 257-266. 
Pries, R., Bomeke, K., Irniger, S., Grundmann, O., and Braus, G.H. (2002). Amino aciddependent Gen $4 p$ stability regulation occurs exclusively in the yeast nucleus. Eukaryot Cell 1, 663-672.

Ravid, T., and Hochstrasser, M. (2008). Diversity of degradation signals in the ubiquitinproteasome system. Nat Rev Mol Cell Biol 9, 679-690.

Ray, A., James, M.K., Larochelle, S., Fisher, R.P., and Blain, S.W. (2009). p27Kip1 inhibits cyclin D-cyclin-dependent kinase 4 by two independent modes. Mol Cell Biol 29, 986-999.

Robbins, J., Dilworth, S.M., Laskey, R.A., and Dingwall, C. (1991). Two interdependent basic domains in nucleoplasmin nuclear targeting sequence: identification of a class of bipartite nuclear targeting sequence. Cell 64, 615-623.

Rohde, J.R., Bastidas, R., Puria, R., and Cardenas, M.E. (2008). Nutritional control via Tor signaling in Saccharomyces cerevisiae. Curr Opin Microbiol 11, 153-160.

Rohde, J.R., Campbell, S., Zurita-Martinez, S.A., Cutler, N.S., Ashe, M., and Cardenas, M.E. (2004). TOR controls transcriptional and translational programs via Sap-Sit4 protein phosphatase signaling effectors. Mol Cell Biol 24, 8332-8341.

Rosenblum, J.S., Pemberton, L.F., Bonifaci, N., and Blobel, G. (1998). Nuclear import and the evolution of a multifunctional RNA-binding protein. J Cell Biol 143, 887-899.

Saiki, R.K., Scharf, S., Faloona, F., Mullis, K.B., Horn, G.T., Erlich, H.A., and Arnheim, N. (1985). Enzymatic amplification of beta-globin genomic sequences and restriction site analysis for diagnosis of sickle cell anemia. Science 230, 1350-1354.

Sattlegger, E., and Hinnebusch, A.G. (2005). Polyribosome binding by GCN1 is required for full activation of eukaryotic translation initiation factor 2 \{alpha\} kinase GCN2 during amino acid starvation. $J$ Biol Chem 280, 16514-16521.

Sattlegger, E., Swanson, M.J., Ashcraft, E.A., Jennings, J.L., Fekete, R.A., Link, A.J., and Hinnebusch, A.G. (2004). YIH1 is an actin-binding protein that inhibits protein kinase GCN2 and impairs general amino acid control when overexpressed. $J$ Biol Chem 279, 29952-29962.

Schlenstedt, G., Saavedra, C., Loeb, J.D., Cole, C.N., and Silver, P.A. (1995). The GTPbound form of the yeast Ran/TC4 homologue blocks nuclear protein import and appearance of poly(A)+ RNA in the cytoplasm. Proc Natl Acad Sci U S A 92, 225229.

Schürch, A., Miozzari, J., and Hutter, R. (1974). Regulation of tryptophan biosynthesis in Saccharomyces cerevisiae: mode of action of 5-methyl-tryptophan and 5-methyltryptophan-sensitive mutants. J Bacteriol 117, 1131-1140.

Shemer, R., Meimoun, A., Holtzman, T., and Kornitzer, D. (2002). Regulation of the transcription factor Gen4 by Pho85 cyclin Pc15. Mol Cell Biol 22, 5395-5404.

Shenton, D., Smirnova, J.B., Selley, J.N., Carroll, K., Hubbard, S.J., Pavitt, G.D., Ashe, M.P., and Grant, C.M. (2006). Global translational responses to oxidative stress impact upon multiple levels of protein synthesis. J Biol Chem 281, 29011-29021.

Shirra, M.K., McCartney, R.R., Zhang, C., Shokat, K.M., Schmidt, M.C., and Arndt, K.M. (2008). A chemical genomics study identifies Snf1 as a repressor of GCN4 translation. J Biol Chem 283, 35889-35898.

Siergiejuk, E., Scott, D.C., Schulman, B.A., Hofmann, K., Kurz, T., and Peter, M. (2009). Cullin neddylation and substrate-adaptors counteract SCF inhibition by the CAND1like protein Lag2 in Saccharomyces cerevisiae. EMBO J 28, 3845-3856.

Sikorski, R.S., and Hieter, P. (1989). A system of shuttle vectors and yeast host strains designed for efficient manipulation of DNA in Saccharomyces cerevisiae. Genetics 122, 19-27. 
Simpson-Lavy, K.J., Oren, Y.S., Feine, O., Sajman, J., Listovsky, T., and Brandeis, M. (2010). Fifteen years of APC/cyclosome: a short and impressive biography. Biochem Soc Trans 38, 78-82.

Smets, B., Ghillebert, R., De Snijder, P., Binda, M., Swinnen, E., De Virgilio, C., and Winderickx, J. (2010). Life in the midst of scarcity: adaptations to nutrient availability in Saccharomyces cerevisiae. Curr Genet 56, 1-32.

Sopko, R., Huang, D., Preston, N., Chua, G., Papp, B., Kafadar, K., Snyder, M., Oliver, S.G., Cyert, M., Hughes, T.R., Boone, C., and Andrews, B. (2006). Mapping pathways and phenotypes by systematic gene overexpression. Mol Cell 21, 319-330.

Sopko, R., Huang, D., Smith, J.C., Figeys, D., and Andrews, B.J. (2007). Activation of the Cdc42p GTPase by cyclin-dependent protein kinases in budding yeast. EMBO J 26, 4487-4500.

Sorokin, A.V., Kim, E.R., and Ovchinnikov, L.P. (2007). Nucleocytoplasmic transport of proteins. Biochemistry (Mosc) 72, 1439-1457.

Sorokin, A.V., Kim, E.R., and Ovchinnikov, L.P. (2009). Proteasome system of protein degradation and processing. Biochemistry (Mosc) 74, 1411-1442.

Southern, E.M. (1975). Detection of specific sequences among DNA fragments separated by gel electrophoresis. J Mol Biol 98, 503-517.

Staschke, K.A., Dey, S., Zaborske, J.M., Palam, L.R., McClintick, J.N., Pan, T., Edenberg, H.J., and Wek, R.C. (2010). Integration of general amino acid control and target of rapamycin (TOR) regulatory pathways in nitrogen assimilation in yeast. $J$ Biol Chem 285, 16893-16911.

Strambio-De-Castillia, C., Niepel, M., and Rout, M.P. (2010). The nuclear pore complex: bridging nuclear transport and gene regulation. Nat Rev Mol Cell Biol 11, 490-501.

Suryadinata, R., Sadowski, M., and Sarcevic, B. (2010). Control of cell cycle progression by phosphorylation of cyclin-dependent kinase (CDK) substrates. Biosci Rep 30, 243255.

Swanson, M.J., Qiu, H., Sumibcay, L., Krueger, A., Kim, S.J., Natarajan, K., Yoon, S., and Hinnebusch, A.G. (2003). A multiplicity of coactivators is required by Gen $4 p$ at individual promoters in vivo. Mol Cell Biol 23, 2800-2820.

Tan, Y.S., Morcos, P.A., and Cannon, J.F. (2003). Pho85 phosphorylates the Glc7 protein phosphatase regulator Glc8 in vivo. J Biol Chem 278, 147-153.

Tarricone, C., Dhavan, R., Peng, J., Areces, L.B., Tsai, L.H., and Musacchio, A. (2001). Structure and regulation of the CDK5-p25(nck5a) complex. Mol Cell 8, 657-669.

Tavernarakis, N., and Thireos, G. (1995). Transcriptional interference caused by GCN4 overexpression reveals multiple interactions mediating transcriptional activation. $\mathrm{Mol}$ Gen Genet 247, 571-578.

Tesfaigzi, J., Smith-Harrison, W., and Carlson, D.M. (1994). A simple method for reusing western blots on PVDF membranes. Biotechniques 17, 268-269.

Towbin, H., Staehelin, T., and Gordon, J. (1979). Electrophoretic transfer of proteins from polyacrylamide gels to nitrocellulose sheets: procedure and some applications. Proc Natl Acad Sci U S A 76, 4350-4354.

Tzamarias, D., Roussou, I., and Thireos, G. (1989). Coupling of GCN4 mRNA translational activation with decreased rates of polypeptide chain initiation. Cell 57, 947-954.

Valenzuela, L., Aranda, C., and Gonzalez, A. (2001). TOR modulates GCN4-dependent expression of genes turned on by nitrogen limitation. J Bacteriol 183, 2331-2334.

Wanke, V., Pedruzzi, I., Cameroni, E., Dubouloz, F., and De Virgilio, C. (2005). Regulation of G0 entry by the Pho80-Pho85 cyclin-CDK complex. EMBO J 24, 4271-4278.

Waters, N.C., Knight, J.P., Creasy, C.L., and Bergman, L.W. (2004). The yeast Pho80-Pho85 cyclin-CDK complex has multiple substrates. Curr Genet 46, 1-9. 
Wek, S.A., Zhu, S., and Wek, R.C. (1995). The histidyl-tRNA synthetase-related sequence in the eIF-2 alpha protein kinase GCN2 interacts with tRNA and is required for activation in response to starvation for different amino acids. Mol Cell Biol 15, 44974506.

Willems, A.R., Lanker, S., Patton, E.E., Craig, K.L., Nason, T.F., Mathias, N., Kobayashi, R., Wittenberg, C., and Tyers, M. (1996). Cdc53 targets phosphorylated G1 cyclins for degradation by the ubiquitin proteolytic pathway. Cell 86, 453-463.

Wilson, W.A., Roach, P.J., Montero, M., Baroja-Fernandez, E., Munoz, F.J., Eydallin, G., Viale, A.M., and Pozueta-Romero, J. (2010). Regulation of glycogen metabolism in yeast and bacteria. FEMS Microbiol Rev.

Wilson, W.A., Wang, Z., and Roach, P.J. (2005). Regulation of yeast glycogen phosphorylase by the cyclin-dependent protein kinase Pho85p. Biochem Biophys Res Commun 329, 161-167.

Woodcock, D.M., Crowther, P.J., Doherty, J., Jefferson, S., DeCruz, E., Noyer-Weidner, M., Smith, S.S., Michael, M.Z., and Graham, M.W. (1989). Quantitative evaluation of Escherichia coli host strains for tolerance to cytosine methylation in plasmid and phage recombinants. Nucleic Acids Res 17, 3469-3478.

Wout, P.K., Sattlegger, E., Sullivan, S.M., and Maddock, J.R. (2009). Saccharomyces cerevisiae Rbg1 protein and its binding partner Gir2 interact on polyribosomes with Gcn1. Eukaryot Cell 8, 1061-1071.

Wu, D., Dou, X., Hashmi, S.B., and Osmani, S.A. (2004). The Pho80-like cyclin of Aspergillus nidulans regulates development independently of its role in phosphate acquisition. J Biol Chem 279, 37693-37703.

Wysocki, R., Javaheri, A., Kristjansdottir, K., Sha, F., and Kron, S.J. (2006). CDK Pho85 targets CDK inhibitor Sic1 to relieve yeast G1 checkpoint arrest after DNA damage. Nat Struct Mol Biol 13, 908-914.

Yang, R., Wek, S.A., and Wek, R.C. (2000). Glucose limitation induces GCN4 translation by activation of Gen2 protein kinase. Mol Cell Biol 20, 2706-2717.

Yang, Z., Geng, J., Yen, W.L., Wang, K., and Klionsky, D.J. (2010). Positive or negative roles of different cyclin-dependent kinase Pho85-cyclin complexes orchestrate induction of autophagy in Saccharomyces cerevisiae. Mol Cell 38, 250-264.

Zaman, S., Lippman, S.I., Zhao, X., and Broach, J.R. (2008). How Saccharomyces responds to nutrients. Annu Rev Genet 42, 27-81.

Zhang, F., Sumibcay, L., Hinnebusch, A.G., and Swanson, M.J. (2004). A triad of subunits from the Gal11/tail domain of Srb mediator is an in vivo target of transcriptional activator Gcn4p. Mol Cell Biol 24, 6871-6886.

Zhang, Z., and Dietrich, F.S. (2005). Mapping of transcription start sites in Saccharomyces cerevisiae using 5' SAGE. Nucleic Acids Res 33, 2838-2851.

Zou, J., Friesen, H., Larson, J., Huang, D., Cox, M., Tatchell, K., and Andrews, B. (2009). Regulation of cell polarity through phosphorylation of Bni4 by Pho85 G1 cyclindependent kinases in Saccharomyces cerevisiae. Mol Biol Cell 20, 3239-3250. 


\section{Acknowledgements}

First of all I would like to thank Prof. Dr. Gerhard Braus for giving me the opportunity to conduct my Ph.D. research in his department. I am grateful for his supervision, support, and discussions and for enabling me to participate in national and international conferences to present our data. Furthermore, I highly appreciate the great working conditions in the department.

Moreover, I would like to thank PD Dr. Stefan Irniger for being the co-referent of this thesis.

Many thanks go to Dr. Oliver Valerius and Dr. Lars Fichtner for a lot of helpful discussions, hints and ideas.

Special thanks go to Katharina „Kate“ Tabke for her contributions during her diploma thesis and the endless discussions. I really enjoyed working with you!

I also want to thank the present and former members of Lab 115.

Malte, Claudia, Katrin, Eva, Verena, Britta, Nicole.

Furthermore, I thank all the present and former members of the department for their help, hints and the great working atmosphere!

Special thanks goes to Dr. Özgür Bayram. Cok teşekkürler!

I also wish to thank Rebekka, Martin, Jenny, Olli and the „Seiler“ Lab: Danni, Anne, Bine and Yvonne! You all are great!

Furthermore, I want to thank Suse for her endless support in the last years.

My deepest thank go to my friends, especially those who tried to get in touch with me, and to my family. Without your endless support this would not have been written!

\section{VIELEN DANK!}




\section{CURRICULUM VITAE}

\section{Florian Schulze}

Anschrift:

Kurze-Geismar-Str. 23

37073 Göttingen

Geburtsdatum:

06.01 .1979

Geburtsort:

Uslar

Staatsangehörigkeit:

deutsch

September 2005 - September 2010

Doktorarbeit am Institut für Mikrobiologie und Genetik der Georg-August-Universität Göttingen in der Abteilung für molekulare Mikrobiologie und Genetik (Prof. G. Braus)

Thema: The role of Pcl5p and Pcl7p in the Gen4p stability regulation of Saccharomyces cerevisiae

Oktober 1999 - Mai 2005

Studium der Biologie an der Georg-August-Universität Göttingen

Abschluss: Diplom Biologie

Hauptfach: Mikrobiologie

1. Nebenfach: Humangenetik

2. Nebenfach: Chemie

Thema der Diplomarbeit:

FLO11-Transkription und Chromatinstruktur-

regulierende Aktivitäten in Saccharomyces cerevisiae

Oktober 1998 - September 1999

Studium der Chemie an

der Georg-August-Universität Göttingen

August 1991 - Juni 1998

Felix-Klein-Gymnasium, Göttingen

$1989-1991$

Orientierungsstufe, Lutherschule, Göttingen

$1985-1989$

Grundschule, Lohbergschule, Göttingen

Göttingen, den 20.09.2010 

